\title{
LOCAL SOLVABILITY OF ELLIPTIC EQUATIONS OF EVEN ORDER WITH HÖLDER COEFFICIENTS
}

\author{
MARÍA AMELIA MUSCHIETTI ${ }^{\dagger}$ AND FEDERICO TOURNIER
}

A la memoria de María Amelia, con muchísimo cariño.

\begin{abstract}
We consider elliptic equations of order $2 m$ with Hölder coefficients. We show local solvability of the Dirichlet problem with $m$ conditions on the boundary of the upper half space. First we consider local solvability in free space and then we treat the boundary case. Our method is based on applying the operator to an approximate solution and iterating in the Hölder spaces. A priori estimates for the approximate solution is the essential part of the paper.
\end{abstract}

\section{INTRODUCTION}

This paper is about local solvability of elliptic equations of order $2 m$ with Hölder coefficients. We consider elliptic operators $L u(x)=\sum_{|\alpha| \leq 2 m} a_{\alpha}(x) D^{\alpha} u(x)$, where the coefficients $a_{\alpha}(x)$ are Hölder continuous and complex valued.

The purpose of this paper is to solve in a direct way two types of problems. First, we treat the local problem in free space and we prove

Theorem. Assume the operator $L$ satisfies $(2.3),(2.1)$ and $(2.2)$ Then there exists $\epsilon>0$ depending on $K_{0}, \lambda$ and $n$ so that given $f \in C^{\delta}\left(\overline{B_{\epsilon}}\right)$, there exists $u \in$ $C^{2 m+\delta}\left(B_{\epsilon}\right)$ such that $L u=f$ in $B_{\frac{\epsilon}{2}}$.

This is Theorem 3.8

And secondly, we treat the local problem in half space with $m$ boundary conditions. Specifically, we prove

Theorem. Let the operator $L$ satisfy 2.3 , (2.1) and (2.2). In addition assume that $(2.7)$ holds. Then there exists $\epsilon>0$ depending on $K_{0}$ and $\lambda$ so that given $f \in C^{\delta}\left(B_{\epsilon}^{+}\right)$, there exists $u \in C^{2 m+\delta}\left(B_{\epsilon}^{+}\right)$such that $L u=f$ in $B_{\frac{\epsilon}{2}}^{+}$and $u$ satisfies the $m$ boundary conditions, $u\left(x^{\prime}, 0\right)=0, \frac{\partial u\left(x^{\prime}, 0\right)}{\partial x_{n}}=0, \ldots, \frac{\partial^{m-1} u\left(x^{\prime}, 0\right)}{\partial x_{n}^{m-1}}=0$.

This is Theorem 4.19.

2010 Mathematics Subject Classification. 35B45, 35C15, 35G15.

Key words and phrases. Higher order elliptic equations; A priori estimates; Hölder coefficients; Boundary estimates.

${ }^{\dagger}$ Deceased, 2016. 
We have proved similar results in [7, where we treated the global problem via Schauder estimates and the continuity method with restrictions on the lower order terms.

One advantage of this direct method is that a solution is shown to exist locally with no restrictions on the lower order terms. Our motivation is that we can use these results to treat the problem in a bounded domain by localizing via partition of unity.

There is a very vast literature concerning this problem but our inspiration came basically from an old and classical paper of Agmon, Douglis and Nirenberg [1] in which they treat the Dirichlet problem in a bounded domain and they impose restrictions on the regularity of the lower order terms. Our work differs in the method of proof and in that our result imposes no restrictions on lower terms. Essential to their work is a representation formula for the constant coefficient equation. This representation formula comes in turn as a result of a Radon type transformation due to F. John [5]. We have also relied for some of the basic estimates on the book [6].

In all of the estimates we will use the letter $C$ to denote a constant that depends only on structure. This means that $C$ can depend on the ellipticity constant $\lambda$, on the dimension $n$, and on the fixed constants $\delta$ and $K_{0}$ introduced below.

\section{Preliminaries}

Let $\epsilon>0$. All our estimates will take place in $B_{\epsilon}(0)$, from now on $B_{\epsilon}$.

Throughout the paper, $\eta_{\epsilon}$ will be a function in $C_{0}^{\infty}\left(B_{\epsilon}\right)$ such that $\eta_{\epsilon}=1$ in $B_{\frac{\epsilon}{2}}$, while $\varphi$ will be a function in $C_{0}^{\infty}\left(B_{2}\right)$ such that $\varphi=1$ in $B_{1}$.

We will frequently consider the truncation $\varphi\left(\frac{\xi}{R}\right)$ with $R \rightarrow \infty$.

We fix two real numbers $\delta$ and $\beta$ such that $0<\delta<\beta \leq 1$.

We consider operators of the form

$$
L u(x)=\sum_{|\alpha| \leq 2 m} a_{\alpha}(x) D^{\alpha} u(x),
$$

where $x \in \mathbb{R}^{n}$ and $a_{\alpha}(x) \in \mathbf{C}$.

About the coefficients $a_{\alpha}$ we will assume that

$$
\left|a_{\alpha}(x)-a_{\alpha}(y)\right| \leq K_{\epsilon, \beta}|x-y|^{\beta}
$$

for all $x, y \in B_{\epsilon}(0)$ and for any multi-index $\alpha$.

We notice that $K_{\epsilon, \delta} \leq \epsilon^{\beta-\delta} K_{\epsilon, \beta}$. Hence in our estimates we will use $\mid a_{\alpha}(x)-$ $a_{\alpha}(y)\left|\leq K_{\epsilon, \delta}\right| x-\left.y\right|^{\delta}$ for all $x, y \in B_{\epsilon}(0)$ and at the end use $K_{\epsilon, \delta} \leq \epsilon^{\beta-\delta} K_{\epsilon, \beta}$. This last constant we can make as small as we need by choosing $\epsilon$ small enough.

Define $\left|a_{\alpha}\right|_{0, B_{\epsilon}}=\sup \left\{\left|a_{\alpha}(x)\right|: x \in B_{\epsilon}\right\}$.

We assume that

$$
K_{0}:=\sum_{|\alpha| \leq 2 m}\left|a_{\alpha}\right|_{0, B_{\epsilon}}<\infty
$$


Let us write the characteristic polynomial of the equation by

$$
p(x, i \xi)=\sum_{|\alpha| \leq 2 m} a_{\alpha}(x)(i \xi)^{\alpha} .
$$

We will assume the ellipticity condition

$$
|p(x, i \xi)| \geq \lambda(1+|\xi|)^{2 m}
$$

for all $x \in \mathbb{R}^{n}$ and for all $\xi \in \mathbb{R}^{n}$.

A crucial consequence of the ellipticity assumption are the following estimates.

Lemma 2.1. There exists a constant $C$ depending on $K_{0}, \lambda$ and $|\gamma|$ such that

$$
\left|D_{\xi}^{\gamma} \frac{1}{p(x, i \xi)}\right| \leq C \frac{1}{(1+|\xi|)^{2 m+|\gamma|}}
$$

for all $x \in B_{\epsilon}$, all $\xi \in \mathbb{R}^{n}$, and all $\gamma$.

More generally, we note also that setting $a(x, y, \xi)=\frac{p(x, i \xi)-p(y, i \xi)}{p(y, i \xi)}$ we have

$$
\left|D_{\xi}^{\gamma} a(x, y, \xi)\right| \leq C K_{\epsilon, \delta} \frac{|x-y|^{\delta}}{(1+|\xi|)^{|\gamma|}},
$$

which holds for all $x, y \in B_{\epsilon}$, all $\xi \in \mathbb{R}^{n}$, and all $\gamma$.

Proof. Let $f(t, \xi)=\sum_{|\alpha| \leq 2 m} a_{\alpha}(x)(i \xi)^{\alpha} t^{2 m-\alpha}=t^{2 m} p\left(x, \frac{i \xi}{t}\right)$.

We have $|f(t, x)| \geq \lambda\left(t^{2 m}+|\xi|^{2 m}\right)$ for all $(t, \xi) \in \mathbb{R}^{n}$.

Let $g(t, \xi)=\frac{1}{f(t, \xi)}$. Note that $g(s t, s \xi)=s^{-2 m} g(t, \xi)$ for $s>0$, and there exists a constant $C$ depending on $K_{0}$ such that $\left|D_{\xi}^{\alpha} g(t, \xi)\right| \leq C$ for all $(t, \xi) \in \mathbb{R}^{n+1}$ such that $t^{2}+|\xi|^{2}=1$.

It follows that $\left|D_{\xi}^{\gamma} g(t, \xi)\right| \leq C\left(t^{2}+|\xi|^{2}\right)^{-\frac{(2 m+\mid \gamma)}{2}}$ for all $(t, \xi) \in \mathbb{R}^{n+1}$. In particular, we get $\left|D_{\xi}^{\gamma} \frac{1}{p(x, i \xi)}\right| \leq C \frac{1}{(1+|\xi|)^{2 m+|\gamma|}}$.

The proof of the other inequality is similar. Note that the same proof as above gives that for $|\alpha| \leq 2 m$,

$$
\left|D_{\xi}^{\gamma} \frac{\left(a_{\alpha}(x)-a_{\alpha}(y)\right)(i \xi)^{\alpha}}{p(y, i \xi)}\right| \leq C K_{\epsilon, \delta} \frac{|x-y|^{\delta}}{(1+|\xi|)^{2 m+|\gamma|-\mid \alpha}} \leq C K_{\epsilon, \delta} \frac{|x-y|^{\delta}}{(1+|\xi|)^{|\gamma|}}
$$

and the inequality follows.

The above estimates suffice to treat the problem in free space.

We now introduce the concepts we need in order to treat the problem in half space.

We assume that the dimension $n \geq 2$. 
We emphasize the last coordinate by writing

$$
\begin{aligned}
p\left(x, i \xi^{\prime}, i \xi_{n}\right) & =\sum_{k=0}^{2 m}\left(i \xi_{n}\right)^{2 m-k} \sum_{\left|\alpha^{\prime}\right| \leq k} a_{\alpha^{\prime}}(x)\left(i \xi^{\prime}\right)^{\alpha^{\prime}} \\
& =a(x)\left(i \xi_{n}\right)^{2 m}+\sum_{k=1}^{2 m}\left(i \xi_{n}\right)^{2 m-k} \sum_{\left|\alpha^{\prime}\right| \leq k} a_{\alpha^{\prime}}(x)\left(i \xi^{\prime}\right)^{\alpha^{\prime}}
\end{aligned}
$$

Note that

$$
|a(x)|=|p(x, 0, i)| \geq \lambda
$$

for all $x \in B_{\epsilon}$.

Notice that by ellipticity, the polynomial $p\left(x, i \xi^{\prime}, z\right)$ as a polynomial in the complex variable $z$ has no pure imaginary roots.

Write $p\left(x, i \xi^{\prime}, z\right)=0, z=z\left(x, \xi^{\prime}\right)$ to denote the roots.

By ellipticity and the $K_{0}$ bound it follows that each root satisfies

Lemma 2.2. There exists a constant $C$ depending on $K_{0}$ and $\lambda$ such that for all $\xi^{\prime} \in \mathbb{R}^{n-1}$ and all $x \in B_{\epsilon}$ we have

$$
\left|\operatorname{Re}\left(z\left(x, \xi^{\prime}\right)\right)\right| \geq C\left(1+\left|\xi^{\prime}\right|\right)
$$

and

$$
\left|z\left(x, \xi^{\prime}\right)\right| \leq C\left(1+\left|\xi^{\prime}\right|\right) .
$$

Proof. Fix $x \in B_{\epsilon}$ and $\xi^{\prime} \in \mathbb{R}^{n-1}$ and let $p(z):=p\left(x, i \xi^{\prime}, z\right)$. Assume $p(z)=0$ and write $z=a+i b$.

We have

$$
\begin{aligned}
\lambda\left(1+\left|\xi^{\prime}\right|^{2 m}+b^{2 m}\right) & \leq|p(i b)|=|p(a+i b)-p(i b)|=\left|\int_{0}^{1} \frac{d}{d s} p(s a+i b) d s\right| \\
& =\left|a \int_{0}^{1} p^{\prime}(s a+i b) d s\right| \leq|a| \max \left\{\left|p^{\prime}(s a+i b)\right|: s \in[0,1]\right\} .
\end{aligned}
$$

Write $p(z)=\sum_{k=0}^{2 m} a_{k} z^{k}$ and $p^{\prime}(z)=\sum_{k=1}^{2 m} k a_{k} z^{k-1}$ and note that $\left|a_{k}\right| \leq C(1+$ $\left.|\xi|^{2 m-k}\right)$.

Hence, $\left|p^{\prime}(s a+i b)\right| \leq C \sum_{k=1}^{2 m} k\left|a_{k}\right| s a+\left.i b\right|^{k-1} \leq C \sum_{k=1}^{2 m}\left(1+\left|\xi^{\prime}\right|^{2 m-k}\right)\left(|a|^{k-1}+\right.$ $\left.|b|^{k-1}\right)$.

Therefore,

$$
\lambda\left(1+\left|\xi^{\prime}\right|^{2 m}+b^{2 m}\right) \leq C|a| \sum_{k=1}^{2 m}\left(1+\left|\xi^{\prime}\right|^{2 m-k}\right)\left(|a|^{k-1}+|b|^{k-1}\right) .
$$

Suppose that $|a| \leq 1+\left|\xi^{\prime}\right|$. Then,

$$
\begin{aligned}
|a| \sum_{k=1}^{2 m}\left(1+\left|\xi^{\prime}\right|^{2 m-k}\right)\left(|a|^{k-1}+|b|^{k-1}\right) & \leq C|a| \sum_{k=1}^{2 m}\left(1+\left|\xi^{\prime}\right|^{2 m-k}\right)\left(1+\left|\xi^{\prime}\right|^{k-1}+|b|^{k-1}\right) \\
& \leq C|a|\left(1+\left|\xi^{\prime}\right|^{2 m-1}+|b|^{2 m-1}\right) .
\end{aligned}
$$


Hence,

proving the first part.

$$
|a| \geq \frac{\lambda\left(1+\left|\xi^{\prime}\right|^{2 m}+|b|^{2 m}\right)}{C\left(1+\left|\xi^{\prime}\right|^{2 m-1}+|b|^{2 m-1}\right)} \geq C\left(1+\left|\xi^{\prime}\right|\right),
$$

Next, if $p(z)=0$ then $a_{2 m} z^{2 m}=-\sum_{k=0}^{2 m-1} a_{k} z^{k}$. Hence

$$
z=-\frac{1}{a_{2 m}} \sum_{k=0}^{2 m-1} \frac{a_{k}}{z^{2 m-k-1}} .
$$

Since $|z| \geq|\operatorname{Re}(z)| \geq C\left(1+\left|\xi^{\prime}\right|\right)$ and $\left|a_{k}\right| \leq C\left(1+\left|\xi^{\prime}\right|\right)^{2 m-k}$, and $\left|a_{2 m}\right| \geq \lambda$, we get

$$
|z| \leq C\left(1+\left|\xi^{\prime}\right|\right) \text {. }
$$

We will need two facts about homogeneous polynomials of degree $2 m$, for $\xi \in \mathbb{R}^{n}$ and $n \geq 3$.

Let $P(x, i \xi)=\sum_{|\alpha|=2 m} a_{\alpha}(x)(i \xi)^{\alpha}$ and assume that $\left.|P(x, i \xi) \geq \lambda| \xi\right|^{2 m}$ for all $\xi \in \mathbb{R}^{n}$.

Notice that $P\left(x, i \xi^{\prime}, z\right) \neq 0$ for $z=i \xi_{n}, \xi_{n} \in \mathbb{R}, \xi^{\prime} \in \mathbb{R}^{n-1}$.

Let $\left\{Z_{k}\left(x, \xi^{\prime}\right): k=1, \ldots, 2 m\right\}$ denote the roots of $P\left(x, i \xi^{\prime}, Z\right)=0$.

Noting that $Z_{k}\left(x, \xi^{\prime}\right)=-Z_{j(k)}\left(x,-\xi^{\prime}\right)$, it follows that exactly $m$ of the roots have positive real part for each $\xi^{\prime}$. Denote these roots by $\left\{Z_{k}^{+}(x, \xi): k=1, \ldots, m\right\}$ and by $\left\{Z_{k}^{-}(x, \xi): k=1, \ldots, m\right\}$ the $m$ roots with negative real part.

Then, we can factor

$$
P\left(x, i \xi^{\prime}, z\right)=a(x) \prod_{k=1}^{m}\left(z-Z_{k}^{+}(x, \xi)\right) \prod_{k=1}^{m}\left(z-Z_{k}^{-}(x, \xi)\right) .
$$

From the homogeneity $p\left(x, s i \xi^{\prime}, s z\right)=s^{2 m} p\left(x, i \xi^{\prime}, z\right)$, it follows that each root satisfies $Z\left(x, s \xi^{\prime}\right)=s Z\left(x, \xi^{\prime}\right)$.

Write

$$
P^{+}\left(x, i \xi^{\prime}, z\right):=\prod_{k=1}^{m}\left(z-Z_{k}^{+}(x, \xi)\right)=\sum_{k=0}^{m} S_{k}^{+}\left(x, \xi^{\prime}\right) z^{m-k}
$$

and

$$
P^{-}\left(x, i \xi^{\prime}, z\right):=\prod_{k=1}^{m}\left(z-Z_{k}^{-}(x, \xi)\right)=\sum_{k=0}^{m} S_{k}^{-}\left(x, \xi^{\prime}\right) z^{m-k} .
$$

Then we have that

$$
S_{k}^{+-}\left(x, \xi^{\prime}\right) \text { are smooth functions of } \xi^{\prime} \text { for } \xi^{\prime} \in \mathbb{R}^{n-1} \backslash\{0\}
$$

and

$$
S_{k}^{+-}\left(x, s \xi^{\prime}\right)=s^{k} S_{k}^{+-}\left(x, \xi^{\prime}\right)
$$

These results can be found in reference [1].

Next, we continue with the polynomial $p(x, i \xi)=\sum_{|\alpha| \leq 2 m} a_{\alpha}(x)(i \xi)^{\alpha}$. Here, we assume $\xi \in \mathbb{R}^{n}$ and $n \geq 2$.

Let $P(x, i \xi, i t)=\sum_{k=0}^{2 m} a_{\alpha}(x)(i \xi)^{\alpha} t^{2 m-\alpha}$ and note that

$$
|P(x, i \xi, i t)| \geq \lambda\left(t^{2 m}+|\xi|^{2 m}\right)
$$


for all $(\xi, t) \in \mathbb{R}^{n+1}$ and

$$
P(x, s i \xi, s i t)=s^{2 m} P(x, i \xi, i t) .
$$

Denote the roots of $P\left(x, i \xi^{\prime}, z, i t\right)=0$ by $Z_{k}\left(x, \xi^{\prime}, t\right)$ and the roots of $p\left(x, i \xi^{\prime}, z\right)=0$ by $z_{k}\left(x, \xi^{\prime}\right)$.

Since, for $t=1$, we have $P(x, i \xi, i)=p(x, i \xi)$, we get

$$
z_{k}\left(x, \xi^{\prime}\right)=Z_{k}\left(x, \xi^{\prime}, 1\right)
$$

It follows that $m$ of the roots $z_{k}\left(x, i \xi^{\prime}\right)$ have positive real part. As above, let $\left\{z_{k}^{+}(x, \xi): k=1, \ldots, m\right\}$ be the $m$ roots with positive real part and $\left\{z_{k}^{-}(x, \xi)\right.$ : $k=1, \ldots, m\}$ the $m$ roots with negative real part.

We can factor

$$
p\left(x, i \xi^{\prime}, z\right)=a(x) \prod_{k=1}^{m}\left(z-z_{k}^{+}(x, \xi)\right) \prod_{k=1}^{m}\left(z-z_{k}^{-}(x, \xi)\right) .
$$

Let

$$
p^{+}\left(x, i \xi^{\prime}, z\right):=\prod_{k=1}^{m}\left(z-z_{k}^{+}(x, \xi)\right)=\sum_{k=0}^{m} s_{k}^{+}\left(x, \xi^{\prime}\right) z^{m-k}
$$

and

$$
p^{-}\left(x, i \xi^{\prime}, z\right):=\prod_{k=1}^{m}\left(z-z_{k}^{-}(x, \xi)\right)=\sum_{k=0}^{m} s_{k}^{-}\left(x, \xi^{\prime}\right) z^{m-k} .
$$

It follows that

$$
s_{k}^{+-}\left(x, \xi^{\prime}\right)=S_{k}\left(x, \xi^{\prime}, 1\right) .
$$

Hence we can conclude:

Lemma 2.3. $s_{k}^{+-}\left(x, \xi^{\prime}\right)$ are smooth functions of $\xi^{\prime}$ for $\xi^{\prime} \in \mathbb{R}^{n-1}$, and there exists a constant $C$ depending on $K_{0}, \lambda$ and $|\gamma|$ such that

$$
\left|D_{\xi^{\prime}}^{\gamma} s_{k}^{+-}\left(x, \xi^{\prime}\right)\right| \leq C\left(1+\mid \xi^{\prime}\right)^{k-|\gamma|}
$$

for all $x \in B_{\epsilon}$ and for all $\xi^{\prime} \in \mathbb{R}^{n-1}$.

In addition, we need to assume that there exists a constant $C$ depending on $K_{0}$ such that

$$
\left|D_{\xi^{\prime}}^{\gamma} s_{k}^{+-}\left(y, \xi^{\prime}\right)-D_{\xi^{\prime}}^{\gamma} s_{k}^{+-}\left(x, \xi^{\prime}\right)\right| \leq C K_{\epsilon, \delta}\left(1+\mid \xi^{\prime}\right)^{k-|\gamma|}|x-y|^{\delta} .
$$

We believe this hypothesis should be a consequence of a similar estimate for homogeneous polynomials with Hölder coefficients but we could not find a proof. It holds for all the examples we have at hand.

We give a few examples of operators satisfying our hypothesis.

Let $\lambda \leq a_{j}(x) \leq \Lambda$ be $C^{\delta}$ real valued functions, $j=1, \ldots, n-1$ and $a_{n}=1$, and consider the operator

$$
L u(x)=\sum_{k=1}^{n} a_{k}(x) D_{k}^{4} u(x)-i u(x) .
$$


We have $p(x, i \xi)=\sum_{k=1}^{n} a_{k}(x) \xi_{k}^{4}-i$ and $p\left(x, i \xi^{\prime}, z\right)=z^{4}+\sum_{k=1}^{n-1} a_{k}(x) \xi_{k}^{4}-i=$ : $z^{4}+A-i$. The roots with positive real part are

$$
z_{1}^{+}\left(x, \xi^{\prime}\right)=r e^{i \frac{\gamma}{4}}, \quad z_{2}^{+}=-i r e^{i \frac{\gamma}{4}}
$$

and the roots with negative real part are

$$
z_{1}^{-}\left(x, \xi^{\prime}\right)=i r e^{i \frac{\gamma}{4}}, \quad z_{2}^{-}=-r e^{i \frac{\gamma}{4}}
$$

where $r=\left(1+A^{2}\right)^{\frac{1}{8}}$ and $\gamma=\arccos \left(\frac{-A}{\sqrt{1+A^{2}}}\right)$, and $s_{1}^{+}=z_{1}^{+}+z_{2}^{+} s_{2}^{+}=z_{1}^{+} z_{2}^{+}$.

The estimates (2.6) and (2.7) follow by direct computation.

The referee proposed an example with real constant coefficients,

$$
L u(x)=D_{n}^{4} u(x)+4 \sum_{k=1}^{n-1} D_{k}^{2} u(x) D_{n}^{2} u(x)+\sum_{k, l=1}^{n-1} D_{k}^{2} u(x) D_{l}^{2} u(x)+3 u(x) .
$$

We have

$$
p\left(i \xi^{\prime}, i \xi_{n}\right)=\left(i \xi_{n}\right)^{4}-4\left(i \xi_{n}\right)^{2}\left|\xi^{\prime}\right|^{2}+\left|\xi^{\prime}\right|^{4}+3
$$

and

$$
p\left(i \xi^{\prime}, z\right)=z^{4}-4\left|\xi^{\prime}\right|^{2} z^{2}+\left|\xi^{\prime}\right|^{4}+3
$$

Also, we have

$$
p^{+}\left(i \xi^{\prime}, z\right)=z^{2}-z \sqrt{4\left|\xi^{\prime}\right|^{2}+2 \sqrt{\left|\xi^{\prime}\right|^{4}+3}}+\sqrt{\left|\xi^{\prime}\right|^{4}+3}
$$

and

$$
p^{-}\left(i \xi^{\prime}, z\right)=z^{2}+z \sqrt{4\left|\xi^{\prime}\right|^{2}+2 \sqrt{\left|\xi^{\prime}\right|^{4}+3}}+\sqrt{\left|\xi^{\prime}\right|^{4}+3}
$$

and again, the estimates 2.6 can be checked directly, and obviously the estimate (2.7) holds since the coefficients do not depend on $x$.

A slight variation of the above is to assume that $a_{k}(x)$ and $b_{k}(x)$ are Hölder continuous real valued such that $\lambda \leq a_{k}(x) \leq \Lambda$ and $\lambda \leq b_{k}(x) \leq \Lambda$ for all $x$ and for some positive constants $\lambda$ and $\bar{\Lambda}$, and let $b_{k, l}(x)=2 b_{k}(x) b_{l}(x)$ for $k \neq l$ and $b_{k, k}(x)=\left(b_{k}(x)\right)^{2}$, and consider

$$
L u(x)=D_{n}^{4} u(x)+4 \sum_{k=1}^{n-1} a_{k}(x) D_{k}^{2} u(x) D_{n}^{2} u(x)+\sum_{k, l=1}^{n-1} b_{k, l}(x) D_{k}^{2} u(x) D_{l}^{2} u(x)+u(x) .
$$

As above we have

$$
p\left(x, i \xi^{\prime}, z\right)=z^{4}-z^{2} \sum_{k=1}^{n-1} a_{k}(x) \xi_{k}^{2}+\left(\sum_{k=1}^{n-1} b_{k}(x) \xi_{k}^{2}\right)^{2}+1
$$

and

$$
s_{1}^{+}\left(x, \xi^{\prime}\right)=\sqrt{\sum_{k=1}^{n-1} a_{k}(x) \xi_{k}^{2}+2 \sqrt{\left(\sum_{k=1}^{n-1} b_{k}(x) \xi_{k}^{2}\right)^{2}+1}}
$$




$$
s_{1}^{-}\left(x, \xi^{\prime}\right)=-\sqrt{\sum_{k=1}^{n-1} a_{k}(x) \xi_{k}^{2}+2 \sqrt{\left(\sum_{k=1}^{n-1} b_{k}(x) \xi_{k}^{2}\right)^{2}+1}}
$$

and

$$
s_{2}^{+}\left(x, \xi^{\prime}\right)=s_{2}^{-}\left(x, \xi^{\prime}\right)=\sqrt{\left(\sum_{k=1}^{n-1} b_{k}(x) \xi_{k}^{2}\right)^{2}+1},
$$

and one can check (2.7) directly.

\section{LOCAL EXISTENCE IN FREE SPACE}

We will work on the Hölder spaces defined below.

Given $g \in C^{\delta}(\bar{\Omega})$, let

$$
\begin{gathered}
|g|=\max \{|g(x)|: x \in \bar{\Omega}\} \\
{[g]=\max \left\{\frac{|g(x)-g(\bar{x})|}{|x-\bar{x}|^{\delta}}: x, \bar{x} \in \bar{\Omega}\right\},}
\end{gathered}
$$

and

$$
|g|_{\delta ; \Omega}=|g|+[g] .
$$

We will apply these definitions to $\Omega=B_{\epsilon}$ or $\Omega=B_{\epsilon}^{+}$. We set

$$
\left|D^{k} u\right|_{0}=\max \left\{\left|D^{\alpha} u\right|_{0}:|\alpha|=k\right\} \quad \text { and } \quad\left[D^{k} u\right]_{\delta}=\max \left\{\left[D^{\alpha} u\right]_{\delta}:|\alpha|=k\right\},
$$

and define

$$
|u|_{2 m+\delta}=\sum_{k=0}^{2 m}\left|D^{k} u\right|_{0}+\left[D^{2 m} u\right]_{\delta} .
$$

The space $C^{2 m+\delta}(\Omega)=\left\{u:|u|_{2 m+\delta}<\infty\right\}$ is a Banach space.

Our goal in this section is to solve

$$
L u=f
$$

in a small ball $B_{\epsilon}$.

Given $g \in C^{\delta}\left(B_{\epsilon}\right)$, we define the approximate solution $N\left(\eta_{\epsilon} g\right)$ by the following formula. For $x \in B_{\epsilon}$ let

$$
N\left(\eta_{\epsilon} g\right)(x)=\lim _{R \rightarrow \infty} \int g(y) \eta_{\epsilon}(y) \int \varphi\left(\frac{\xi}{R}\right) \frac{e^{i(x-y) \cdot \xi}}{p(y, i \xi)} d \xi d y .
$$

We will find a solution to $L u=f$ of the form $u=N\left(\eta_{\epsilon} g\right)$ for an appropriate $g$.

In fact, if the equation has constant coefficients, then $u=N\left(\eta_{\epsilon} f\right)$ already solves $L u=f$.

We call $u:=N\left(\eta_{\epsilon} g\right)(x)$ and approximate solution to the equation $L u=g$ in $B_{\epsilon}$ since we will show that the $C^{\delta}$ norm of $L u-g$ is small in $B_{\epsilon}$.

Before we proceed with the main result we need to prove some auxiliary estimates. 
3.1. Auxiliary integrals for free space. In this subsection we prove two auxiliary results to be used below.

First, let us a emphasize the basic calculations that will be used several times in the sequel.

Lemma 3.1. Consider, for $|\alpha| \leq 2 m$, the integral

$$
J=\int e^{i(x-y) \cdot \xi} \frac{\xi^{\alpha}}{p(y, i \xi)} \varphi\left(\frac{\xi}{R}\right) d \xi
$$

We have $|J| \leq C \frac{1}{|x-y|^{n-2 m+|\alpha|}}$, for any $|\alpha| \leq 2 m$ and $R>1$. The constant $C$ depends only on $K_{0},|\alpha|$ and $n$.

Proof. Fix $|\alpha| \leq 2 m$. We may assume $n-2 m+|\alpha|>0$. Note that $\left(-\Delta_{\xi}\right)^{k} e^{i(x-y) . \xi}=$ $|x-y|^{2 k} e^{i(x-y) \cdot \xi}$. Fix $k$ such that $2 k \geq n+1$ and integrate by parts to get

$$
\begin{aligned}
& J=\frac{1}{|x-y|^{2 k}} \int e^{i(x-y) \cdot \xi}(-\Delta)^{k}\left(\frac{\xi^{\alpha}}{p(y, i \xi)} \varphi\left(\frac{\xi}{R}\right)\right) d \xi \\
& =\frac{1}{|x-y|^{2 k}} \int e^{i(x-y) \cdot \xi}(-\Delta)^{k}\left(\frac{\xi^{\alpha}}{p(y, i \xi)} \varphi\left(\frac{\xi}{R}\right)\right) \varphi(|x-y| \xi) d \xi \\
& +\frac{1}{|x-y|^{2 k}} \int e^{i(x-y) \cdot \xi}(-\Delta)^{k}\left(\frac{\xi^{\alpha}}{p(y, i \xi)} \varphi\left(\frac{\xi}{R}\right)\right)(1-\varphi(|x-y| \xi)) d \xi \\
& =\frac{1}{|x-y|^{2 k}} \int(-\Delta)^{k}\left(e^{i(x-y) \cdot \xi} \varphi(|x-y| \xi)\right) \frac{\xi^{\alpha}}{p(y, i \xi)} \varphi\left(\frac{\xi}{R}\right) d \xi \\
& +\frac{1}{|x-y|^{2 k}} \int e^{i(x-y) \cdot \xi}(-\Delta)^{k}\left(\frac{\xi^{\alpha}}{p(y, i \xi)}\right) \varphi\left(\frac{\xi}{R}\right)(1-\varphi(|x-y| \xi)) d \xi \\
& +\frac{1}{|x-y|^{2 k}} \sum_{|\gamma|+|\beta|=2 k, \mid \beta \geq 1} \int e^{i(x-y) \cdot \xi} D^{\gamma}\left(\xi^{\alpha} \frac{1}{p(x, i \xi)}\right) D^{\beta}\left(\varphi\left(\frac{\xi}{R}\right)\right) \\
& \times(1-\varphi(|x-y| \xi)) d \xi \\
& =J_{1}+J_{2}+J_{3} .
\end{aligned}
$$

We estimate each integral as follows, using the first estimate in Lemma 2.1

$$
\begin{aligned}
\left|J_{1}\right| & \leq \int_{|\xi| \leq \frac{2}{|x-y|}} \frac{1}{(1+|\xi|)^{2 m-|\alpha|}} d \xi \leq \frac{C}{|x-y|^{n-2 m+|\alpha|}}, \\
\left|J_{2}\right| & \leq \frac{1}{|x-y|^{2 k}} \int_{|\xi| \geq \frac{1}{|x-y|}} \frac{1}{(1+|\xi|)^{2 m+2 k-|\alpha|}} d \xi \leq \frac{C}{|x-y|^{n-2 m+|\alpha|}}, \\
\left|J_{3}\right| & \leq \frac{1}{|x-y|^{2 k}} \sum_{|\gamma|+|\beta|=2 k, \mid \beta \geq 1} \frac{1}{R^{|\beta|}} \int_{\frac{1}{|x-y|} \leq|\xi| \leq 2 R} \frac{1}{(1+|\xi|)^{2 m+|\gamma|-|\alpha|}} d \xi \\
& \leq \frac{1}{|x-y|^{n-2 m+|\alpha|}} X_{|x-y| \geq \frac{1}{2 R}}
\end{aligned}
$$

where $X$ is the indicator function. It is also important to notice that $J_{3} \rightarrow 0$ as $R \rightarrow \infty$, for $x \neq y$. 
Next, we prove two important bounds that we need in order to continue. Notice that for $|\alpha|=2 m$ the integrand in the integral below is only bounded by $\frac{1}{|x-y|^{n}}$, which is not integrable. However, note that the polynomial is evaluated at $x$ and this allows for a favorable estimate.

Lemma 3.2. Let $|\alpha|=2 m$ and let

$$
I=\int \eta_{\epsilon}(y) \int e^{i(x-y) \cdot \xi} \frac{\xi^{\alpha}}{p(x, i \xi)} \varphi\left(\frac{\xi}{R}\right) d \xi d y .
$$

We have $|I| \leq C$, where $C$ depends only on $K_{0}$ and $n$.

Proof. To prove this, write

$$
I=\int(1-\Delta) \eta_{\epsilon}(y) \int e^{i(x-y) \cdot \xi} \frac{\xi^{\alpha}}{\left(1+|\xi|^{2}\right) p(x, i \xi)} \varphi\left(\frac{\xi}{R}\right) d \xi d y
$$

and note that by Lemma 3.1

$$
|I| \leq C \frac{1}{\epsilon^{2}} \int_{B_{\epsilon}} \frac{1}{|x-y|^{n-2}} d y \leq C
$$

Similarly we have

Lemma 3.3. Let $|\alpha|=2 m$. Let $x, \bar{x} \in B_{\epsilon}, \hat{x}=\frac{x+\bar{x}}{2}$, and $\rho=2|x-\bar{x}|$, so $\rho \leq 4 \epsilon$. Let

$$
I=\int_{B_{\rho}(\hat{x})} \eta_{\epsilon}(y) \int e^{i(x-y) \cdot \xi} \frac{\xi^{\alpha}}{p(x, i \xi)} \varphi\left(\frac{\xi}{R}\right) d \xi d y .
$$

We have $|I| \leq C$, where $C$ depends only on $K_{0}$ and $n$.

Proof. To prove this, write

$$
\begin{aligned}
I= & \int_{B_{\rho}(\hat{x})} \eta_{\epsilon}(y)-\eta_{\epsilon}(x) \int e^{i(x-y) \cdot \xi} \frac{\xi^{\alpha}}{p(x, i \xi)} \varphi\left(\frac{\xi}{R}\right) d \xi d y \\
& +\eta_{\epsilon}(x) \int_{B_{\rho}(\hat{x})} \int e^{i(x-y) \cdot \xi} \frac{\xi^{\alpha}}{p(x, i \xi)} \varphi\left(\frac{\xi}{R}\right) d \xi d y \\
= & A+\eta_{\epsilon}(x) B .
\end{aligned}
$$

We have, by Lemma 3.1 .

$$
|A| \leq C \int_{B_{\rho}(\hat{x})} \frac{\left|\eta_{\epsilon}(y)-\eta_{\epsilon}(x)\right|}{|x-y|^{n}} d y \leq \frac{C}{\epsilon} \int_{B_{\rho}(\hat{x})} \frac{1}{|x-y|^{n-1}} d y \leq C \frac{\rho}{\epsilon} \leq C
$$

and

$$
\begin{aligned}
B= & \int_{B_{\rho}(\hat{x})}(1-\Delta) \int e^{i(x-y) \cdot \xi} \frac{\xi^{\alpha}}{p(x, i \xi)\left(1+|\xi|^{2}\right)} \varphi\left(\frac{\xi}{R}\right) d \xi d y \\
= & \int_{B_{\rho}(\hat{x})} \int e^{i(x-y) \cdot \xi} \frac{\xi^{\alpha}}{p(x, i \xi)\left(1+|\xi|^{2}\right)} \varphi\left(\frac{\xi}{R}\right) d \xi d y \\
& -\int_{\partial B_{\rho}(\hat{x})}\left\langle\nabla_{y} \int e^{i(x-y) \cdot \xi} \frac{\xi^{\alpha}}{p(x, i \xi)\left(1+|\xi|^{2}\right)} \varphi\left(\frac{\xi}{R}\right) d \xi, \frac{y-\hat{x}}{|y-\hat{x}|}\right\rangle d S_{y} \\
= & B_{1}+B_{2} .
\end{aligned}
$$


We can estimate again by Lemma 3.1

$$
\left|B_{1}\right| \leq C \int_{B_{\rho}(\hat{x})} \frac{1}{|x-y|^{n-2}} d y \leq C \rho^{2}
$$

and

$$
\left|B_{2}\right| \leq \int_{\partial B_{\rho}(\hat{x})} \frac{1}{|x-y|^{n-1}} d S_{y} \leq C
$$

Next we show that $N\left(\eta_{\epsilon} g\right)$ has derivatives up to order $2 m$.

Lemma 3.4. The function $u:=N\left(\eta_{\epsilon} g\right) \in C^{2 m}\left(B_{\epsilon}\right)$. In fact,

$$
D^{\alpha} N\left(\eta_{\epsilon} g\right)(x)=\lim _{R \rightarrow \infty} \int g(y) \eta_{\epsilon}(y) \int \varphi\left(\frac{\xi}{R}\right) \frac{(i \xi)^{\alpha} e^{i(x-y) \cdot \xi}}{p(y, i \xi)} d \xi d y
$$

for any multi-index $\alpha,|\alpha| \leq 2 m$.

Proof. Let $|\alpha|=2 \mathrm{~m}$. We show that the above limit exists and is uniform in $x$.

Write

$$
\int g(y) \eta_{\epsilon}(y) \int \varphi\left(\frac{\xi}{R}\right) \frac{(i \xi)^{\alpha} e^{i(x-y) \cdot \xi}}{p(y, i \xi)} d \xi d y=I_{R}+g(x) I I_{R}+g(x) I I I_{R},
$$

where

$$
\begin{aligned}
I_{R} & =\int(g(y)-g(x)) \eta_{\epsilon}(y) \int \varphi\left(\frac{\xi}{R}\right) \frac{(i \xi)^{\alpha} e^{i(x-y) \cdot \xi}}{p(y, i \xi)} d \xi d y \\
I I_{R} & =\int \eta_{\epsilon}(y) \int \varphi\left(\frac{\xi}{R}\right) \frac{(i \xi)^{\alpha} e^{i(x-y) \cdot \xi}}{p(x, i \xi)} d \xi d y \\
I I I_{R} & =\int \eta_{\epsilon}(y) \int \varphi\left(\frac{\xi}{R}\right)(i \xi)^{\alpha} e^{i(x-y) \cdot \xi}\left(\frac{1}{p(y, i \xi)}-\frac{1}{p(x, i \xi)}\right) d \xi d y .
\end{aligned}
$$

Let $k$ be an integer such that $2 k \geq n+1$ and let

$$
\begin{aligned}
I & =\int \frac{(g(y)-g(x)) \eta_{\epsilon}(y)}{|x-y|^{2 k}} \int e^{i(x-y) \cdot \xi}\left(-\Delta_{\xi}\right)^{k}\left(\frac{(i \xi)^{\alpha}}{p(y, i \xi)}\right) d \xi d y, \\
I I & =\int(1-\Delta)\left(\eta_{\epsilon}(y)\right) \frac{1}{|x-y|^{2 k}} \int e^{i(x-y) \cdot \xi}\left(-\Delta_{\xi}\right)^{k}\left(\frac{(i \xi)^{\alpha}}{\left(1+|\xi|^{2}\right) p(x, i \xi)}\right) d \xi d y, \\
I I I & =\int \frac{\eta_{\epsilon}(y)}{|x-y|^{2 k}} \int e^{i(x-y) \cdot \xi}\left(-\Delta_{\xi}\right)^{k}\left((i \xi)^{\alpha}\left(\frac{1}{p(y, i \xi)}-\frac{1}{p(x, i \xi)}\right)\right) d \xi d y .
\end{aligned}
$$

First we show that the integrals converge.

We claim that

$$
\left|\int e^{i(x-y) \cdot \xi}\left(-\Delta_{\xi}\right)^{k}\left(\frac{(i \xi)^{\alpha}}{p(y, i \xi)}\right) d \xi\right| \leq C|x-y|^{2 k-n}
$$


Write

$$
\begin{aligned}
\int e^{i(x-y) \cdot \xi}\left(-\Delta_{\xi}\right)^{k} & \left(\frac{(i \xi)^{\alpha}}{p(y, i \xi)}\right) d \xi \\
= & \int e^{i(x-y) \cdot \xi}\left(-\Delta_{\xi}\right)^{k}\left(\frac{(i \xi)^{\alpha}}{p(y, i \xi)}\right) \eta(|x-y| \xi) d \xi \\
& +\int e^{i(x-y) \cdot \xi}\left(-\Delta_{\xi}\right)^{k}\left(\frac{(i \xi)^{\alpha}}{p(y, i \xi)}\right)(1-\eta(|x-y| \xi)) d \xi \\
= & A+B .
\end{aligned}
$$

Integration by parts gives that

$$
A=\int_{|\xi| \leq 2|x-y|^{-1}}\left(-\Delta_{\xi}\right)^{k}\left(e^{i(x-y) \cdot \xi} \eta(|x-y| \xi)\right) \frac{(i \xi)^{\alpha}}{p(y, i \xi)} d \xi .
$$

Next, note that $\left|\left(-\Delta_{\xi}\right)^{k}\left(e^{i(x-y) \cdot \xi} \eta(|x-y| \xi)\right)\right| \leq C|x-y|^{2 k}$, and hence

$$
\begin{aligned}
|A| & \leq C|x-y|^{2 k}\left|\int_{|\xi| \leq 2|x-y|^{-1}}\left(\frac{(i \xi)^{\alpha}}{p(y, i \xi)}\right) d \xi\right| \\
& \leq C|x-y|^{2 k} \int_{|\xi| \leq 2|x-y|^{-1}} d \xi \leq C|x-y|^{2 k-n} .
\end{aligned}
$$

Using (2.4) we get

$$
|B| \leq C \int_{|\xi| \geq|x-y|^{-1}}(1+|\xi|)^{-2 k} d \xi \leq C|x-y|^{2 k-n}
$$

This proves the claim.

It follows that

$$
|I| \leq \int \frac{|g(y)-g(x)| \eta_{\epsilon}(y)}{|x-y|^{n}} d y \leq C \epsilon^{\delta} .
$$

The convergence of the integral $I I$ is proved in the same way, this time noting that

$$
\left|\int e^{i(x-y) \cdot \xi}\left(-\Delta_{\xi}\right)^{k}\left(\frac{(i \xi)^{\alpha}}{\left(1+|\xi|^{2}\right) p(x, i \xi)}\right) d \xi\right| \leq C|x-y|^{2 k-n+2},
$$

and similarly for the integral $I I I$, this time using (2.5) to estimate

$$
\left|\int e^{i(x-y) \cdot \xi}\left(-\Delta_{\xi}\right)^{k}\left((i \xi)^{\alpha}\left(\frac{1}{p(y, i \xi)}-\frac{1}{p(x, i \xi)}\right)\right) d \xi\right| \leq C|x-y|^{2 k-n+\delta}
$$

Let us now show that $I I I_{R} \rightarrow I I I$ as $R \rightarrow \infty$. The other two are similar.

First, we write

$$
\begin{aligned}
I I I= & \int \frac{\eta_{\epsilon}(y)}{|x-y|^{2 k}} \int e^{i(x-y) \cdot \xi}\left(-\Delta_{\xi}\right)^{k}\left((i \xi)^{\alpha}\left(\frac{1}{p(y, i \xi)}-\frac{1}{p(x, i \xi)}\right)\right) \varphi(|x-y| \xi) d \xi d y \\
& +\int \frac{\eta_{\epsilon}(y)}{|x-y|^{2 k}} \int e^{i(x-y) \cdot \xi}\left(-\Delta_{\xi}\right)^{k}\left((i \xi)^{\alpha}\left(\frac{1}{p(y, i \xi)}-\frac{1}{p(x, i \xi)}\right)\right) \\
= & \times(1-\varphi(|x-y| \xi)) d \xi d y \\
= &
\end{aligned}
$$


and note that

$$
A=\int \frac{\eta_{\epsilon}(y)}{|x-y|^{2 k}} \int\left(-\Delta_{\xi}\right)^{k}\left(e^{i(x-y) \cdot \xi} \varphi(|x-y| \xi)\right)(i \xi)^{\alpha}\left(\frac{1}{p(y, i \xi)}-\frac{1}{p(x, i \xi)}\right) d \xi d y
$$

We have

$$
I I I_{R}=\int \frac{\eta_{\epsilon}(y)}{|x-y|^{2 k}} \int e^{i(x-y) \cdot \xi}\left(-\Delta_{\xi}\right)^{k}\left((i \xi)^{\alpha}\left(\frac{1}{p(y, i \xi)}-\frac{1}{p(x, i \xi)}\right) \varphi\left(\frac{\xi}{R}\right)\right) d \xi d y .
$$

and hence we can write

$$
I I I_{R}=A_{R}+B_{R}+C_{R}
$$

where

$$
\begin{aligned}
A_{R}= & \int \frac{\eta_{\epsilon}(y)}{|x-y|^{2 k}} \int\left(-\Delta_{\xi}\right)^{k}\left(e^{i(x-y) \cdot \xi} \varphi(|x-y| \xi)\right)(i \xi)^{\alpha}\left(\frac{1}{p(y, i \xi)}-\frac{1}{p(x, i \xi)}\right) \\
& \times \varphi\left(\frac{\xi}{R}\right) d \xi d y \\
B_{R}= & \int \frac{\eta_{\epsilon}(y)}{|x-y|^{2 k}} \int e^{i(x-y) \cdot \xi}\left(-\Delta_{\xi}\right)^{k}\left((i \xi)^{\alpha}\left(\frac{1}{p(y, i \xi)}-\frac{1}{p(x, i \xi)}\right)\right) \\
& \quad \times \varphi\left(\frac{\xi}{R}\right)(1-\varphi(|x-y| \xi)) d \xi d y, \\
C_{R}= & \sum_{|\gamma|+|\beta|=2 k, \mid \beta \geq 1} \int \frac{\eta_{\epsilon}(y)}{|x-y|^{2 k}} \int e^{i(x-y) \cdot \xi} D^{\gamma}\left((i \xi)^{\alpha}\left(\frac{1}{p(y, i \xi)}-\frac{1}{p(x, i \xi)}\right)\right) \\
& \quad \times D^{\beta}\left(\varphi\left(\frac{\xi}{R}\right)\right)(1-\varphi(|x-y| \xi)) d \xi d y .
\end{aligned}
$$

Therefore, $\left|I I I_{R}-I I I\right| \leq\left|A-A_{R}\right|+\left|B-B_{R}\right|+\left|C_{R}\right|$. We estimate

$$
\begin{aligned}
\left|A-A_{R}\right| \leq & C \int \eta_{\epsilon}(y)|x-y|^{\delta} \int_{|\xi| \leq \frac{2}{|x-y|}} 1-\varphi\left(\frac{\xi}{R}\right) d \xi d y \\
\leq & C \int \eta_{\epsilon}(y)|x-y|^{\delta} \int_{R \leq|\xi| \leq \frac{2}{|x-y|}} 1 d \xi d y \leq C R^{-\delta} \\
\left|B-B_{R}\right| \leq & C \int \eta_{\epsilon}(y) \frac{|x-y|^{\delta}}{|x-y|^{2 k}} \int_{|\xi| \geq \frac{1}{|x-y|}} \frac{1-\varphi\left(\frac{\xi}{R}\right)}{(1+|\xi|)^{2 k}} d \xi d y \\
\leq & \int_{|x-y| \leq \frac{1}{R}} \frac{|x-y|^{\delta}}{|x-y|^{2 k}} \int_{\frac{1}{|x-y|} \leq|\xi|} \frac{1}{(1+|\xi|)^{2 k}} d \xi d y \\
& +\int_{|x-y| \geq \frac{1}{R}} \frac{1 x-\left.y\right|^{\delta}}{|x-y|^{2 k}} \int_{R \leq|\xi|} \frac{1}{(1+|\xi|)^{2 k}} d \xi d y \\
\leq & C R^{-\delta},
\end{aligned}
$$

and

$$
\left|C_{R}\right| \leq C \int_{|x-y| \geq \frac{1}{2 R}} \frac{|x-y|^{\delta}}{|x-y|^{2 k}} \frac{1}{R^{|\beta|}} \int_{R \leq|\xi| \leq 2 R} \frac{1}{(1+|\xi|)^{|\gamma|}} d \xi d y \leq C R^{-\delta} .
$$


To show that $I I_{R} \rightarrow I I$, first note that

$$
\begin{aligned}
I I_{R}= & \int(1-\Delta) \eta_{\epsilon}(y) \int e^{i(x-y) \cdot \xi}\left(\frac{(i \xi)^{\alpha}}{\left(1+|\xi|^{2}\right) p(x, i \xi)}\right) \varphi\left(\frac{\xi}{R}\right) d \xi d y \\
= & \int(1-\Delta)\left(\eta_{\epsilon}(y)\right) \frac{1}{|x-y|^{2 k}} \int e^{i(x-y) \cdot \xi}\left(-\Delta_{\xi}\right)^{k} \\
& \times\left(\frac{(i \xi)^{\alpha}}{\left(1+|\xi|^{2}\right) p(x, i \xi)} \varphi\left(\frac{\xi}{R}\right)\right) d \xi d y
\end{aligned}
$$

and then we proceed as before.

\subsection{Main results for free space.}

Lemma 3.5. The function defined by $u=N\left(g \eta_{\epsilon}\right)$ is in $C^{2 m+\delta}\left(B_{\epsilon}\right)$.

Proof. We have shown that $u$ has derivatives up to order $2 m$ and for $|\alpha| \leq 2 m$ we have $D^{\alpha} u(x)=\lim _{R \rightarrow \infty} \int g(y) \eta_{\epsilon}(y) \int \varphi\left(\frac{\xi}{R}\right) \frac{(i \xi)^{\alpha} e^{i(x-y) . \xi}}{p(y, i \xi)} d \xi d y$. Let $|\alpha|=2 m$, and let

$$
I_{R}=\int g(y) \eta_{\epsilon}(y) \int \varphi\left(\frac{\xi}{R}\right) \frac{(i \xi)^{\alpha} e^{i(x-y) \cdot \xi}}{p(y, i \xi)} d \xi d y .
$$

Let $x, \bar{x} \in B_{\epsilon}$.

Write $I_{R}(x)-I_{R}(\bar{x})=A+B$, where

$$
\begin{aligned}
A & =\int g(y) \eta_{\epsilon}(y) \int\left(e^{i(x-y) \cdot \xi}-e^{i(\bar{x}-y) \cdot \xi}\right)(i \xi)^{\alpha} \frac{1}{p(\bar{x}, i \xi)} \varphi\left(\frac{\xi}{R}\right) d \xi d y \\
B & =\int g(y) \eta_{\epsilon}(y) \int\left(e^{i(x-y) \cdot \xi}-e^{i(\bar{x}-y) \cdot \xi}\right)(i \xi)^{\alpha}\left(\frac{1}{p(y, i \xi)}-\frac{1}{p(\bar{x}, i \xi)}\right) \varphi\left(\frac{\xi}{R}\right) d \xi d y .
\end{aligned}
$$

Write $A=B_{1}+B_{2}$, where

$$
\begin{aligned}
& B_{1}=\int(g(y)-g(x)) \eta_{\epsilon}(y) \int\left(e^{i(x-y) \cdot \xi}-e^{i(\bar{x}-y) \cdot \xi}\right) \frac{(i \xi)^{\alpha}}{p(\bar{x}, i \xi)} \varphi\left(\frac{\xi}{R}\right) d \xi d y \\
& B_{2}=g(x) \int \eta_{\epsilon}(y) \int\left(e^{i(x-y) \cdot \xi}-e^{i(\bar{x}-y) \cdot \xi}\right) \frac{(i \xi)^{\alpha}}{p(\bar{x}, i \xi)} \varphi\left(\frac{\xi}{R}\right) d \xi d y
\end{aligned}
$$

Write $B_{1}=C_{1}-C_{2}+C_{3}$, where

$$
\begin{aligned}
C_{1} & =\int_{B_{\rho}(\hat{x})}(g(y)-g(x)) \eta_{\epsilon}(y) \int e^{i(x-y) \cdot \xi} \frac{(i \xi)^{\alpha}}{p(\bar{x}, i \xi)} \varphi\left(\frac{\xi}{R}\right) d \xi d y, \\
C_{2} & =\int_{B_{\rho}(\hat{x})}(g(y)-g(x)) \eta_{\epsilon}(y) \int e^{i(\bar{x}-y) \cdot \xi} \frac{(i \xi)^{\alpha}}{p(\bar{x}, i \xi)} \varphi\left(\frac{\xi}{R}\right) d \xi d y \\
C_{3} & =\int_{R^{n} \backslash B_{\rho}(\hat{x})}(g(y)-g(x)) \eta_{\epsilon}(y) \int\left(e^{i(x-y) \cdot \xi}-e^{i(\bar{x}-y) \cdot \xi}\right) \frac{(i \xi)^{\alpha}}{p(\bar{x}, i \xi)} \varphi\left(\frac{\xi}{R}\right) d \xi d y .
\end{aligned}
$$


We have, using Lemma 3.1 , that $\left|C_{1}\right| \leq C \int_{B_{\rho}(\hat{x})}|y-x|^{\delta} \frac{1}{|y-x|^{n}} d y \leq C \rho^{\delta} \leq C|x-\bar{x}|^{\delta}$ and $C_{2}=D_{1}+D_{2}$, where

$$
\begin{aligned}
& D_{1}=\int_{B_{\rho}(\hat{x})}(g(y)-g(\bar{x})) \eta_{\epsilon}(y) \int e^{i(\bar{x}-y) \cdot \xi} \frac{(i \xi)^{\alpha}}{p(\bar{x}, i \xi)} \varphi\left(\frac{\xi}{R}\right) d \xi d y \\
& D_{2}=(g(x)-g(\bar{x})) \int_{B_{\rho}(\hat{x})} \eta_{\epsilon}(y) \int e^{i(\bar{x}-y) \cdot \xi} \frac{(i \xi)^{\alpha}}{p(\bar{x}, i \xi)} \varphi\left(\frac{\xi}{R}\right) d \xi d y .
\end{aligned}
$$

Estimate $\left|D_{1}\right| \leq C \rho^{\delta} \leq C|x-\bar{x}|^{\delta}$ just like $C_{1}$ and

$$
\left|D_{2}\right| \leq C|x-\bar{x}|^{\delta}\left|\int_{B_{\rho}(\hat{x})} \eta_{\epsilon}(y) \int e^{i(\bar{x}-y) \cdot \xi} \frac{(i \xi)^{\alpha}}{p(\bar{x}, i \xi)} \varphi\left(\frac{\xi}{R}\right) d \xi d y\right| \leq C|x-\bar{x}|^{\delta}
$$

by Lemma 3.3. Next,

$$
C_{3}=\int_{R^{n} \backslash B_{\rho}(\hat{x})}(g(y)-g(x)) \eta_{\epsilon}(y) \int i \int_{0}^{1} e^{i\left(x_{s}-y\right) \cdot \xi} d s(x-\bar{x}) \cdot \xi \frac{(i \xi)^{\alpha}}{p(\bar{x}, i \xi)} \varphi\left(\frac{\xi}{R}\right) d \xi d y,
$$

and we have, by Lemma 3.1

$$
\begin{aligned}
\left|C_{3}\right| & \leq C|x-\bar{x}| \int_{0}^{1} \int_{R^{n} \backslash B_{\rho}(\hat{x})}|y-x|^{\delta} \frac{1}{\left|x_{s}-y\right|^{n+1}} d y d s \\
& \leq C|x-\bar{x}| \int_{R^{n} \backslash B_{\rho}(\hat{x})}|y-x|^{\delta} \frac{1}{|x-y|^{n+1}} d y \\
& \leq C|x-\bar{x}| \rho^{\delta-1} \leq C|x-\bar{x}|^{\delta},
\end{aligned}
$$

finishing the estimation of $B_{1}$. Now,

$$
B_{2}=\int(1-\Delta) \eta_{\epsilon}(y) \int\left(e^{i(x-y) \cdot \xi}-e^{i(\bar{x}-y) \cdot \xi}\right) \frac{(i \xi)^{\alpha}}{\left(1+|\xi|^{2}\right) p(\bar{x}, i \xi)} \varphi\left(\frac{\xi}{R}\right) d \xi d y
$$

and by Lemma 3.1

$$
\left|B_{2}\right| \leq C|x-\bar{x}| \frac{1}{\epsilon^{2}} \int_{B_{\epsilon}} \int_{0}^{1} \frac{1}{\left|x_{s}-y\right|^{n-1}} d s d y \leq C \frac{|x-\bar{x}|}{\epsilon} \leq \frac{C}{\epsilon^{\delta}}|x-\bar{x}|^{\delta} .
$$

This finishes the estimation of the term $A$.

Write $B=C_{1}-C_{2}+C_{3}$, where

$$
\begin{array}{r}
C_{1}=\int_{B_{\rho}(\hat{x})} g(y) \eta_{\epsilon}(y) \int e^{i(x-y) \cdot \xi}(i \xi)^{\alpha}\left(\frac{1}{p(y, i \xi)}-\frac{1}{p(\bar{x}, i \xi)}\right) \varphi\left(\frac{\xi}{R}\right) d \xi d y, \\
C_{2}=\int_{B_{\rho}(\hat{x})} g(y) \eta_{\epsilon}(y) \int e^{i(\bar{x}-y) \cdot \xi}(i \xi)^{\alpha}\left(\frac{1}{p(y, i \xi)}-\frac{1}{p(\bar{x}, i \xi)}\right) \varphi\left(\frac{\xi}{R}\right) d \xi d y, \\
C_{3}=\int_{R^{n} \backslash B_{\rho}(\hat{x})} g(y) \eta_{\epsilon}(y) \int\left(e^{i(x-y) \cdot \xi}-e^{i(\bar{x}-y) \cdot \xi}\right)(i \xi)^{\alpha}\left(\frac{1}{p(y, i \xi)}-\frac{1}{p(\bar{x}, i \xi)}\right) \\
\times \varphi\left(\frac{\xi}{R}\right) d \xi d y .
\end{array}
$$


Write $C_{1}=D_{1}+D_{2}$, where

$$
\begin{aligned}
& D_{1}=\int_{B_{\rho}(\hat{x})}(g(y)-g(x)) \eta_{\epsilon}(y) \int e^{i(x-y) \cdot \xi}(i \xi)^{\alpha}\left(\frac{1}{p(y, i \xi)}-\frac{1}{p(\bar{x}, i \xi)}\right) \varphi\left(\frac{\xi}{R}\right) d \xi d y \\
& D_{2}=g(x) \int_{B_{\rho}(\hat{x})} \eta_{\epsilon}(y) \int e^{i(x-y) \cdot \xi}(i \xi)^{\alpha}\left(\frac{1}{p(y, i \xi)}-\frac{1}{p(\bar{x}, i \xi)}\right) \varphi\left(\frac{\xi}{R}\right) d \xi d y,
\end{aligned}
$$

and we estimate

$$
\left|D_{1}\right| \leq C \int_{B_{\rho}(\hat{x})}|y-x|^{\delta} \frac{1}{|x-y|^{n}} d y \leq C \rho^{\delta} \leq C|x-\bar{x}|^{\delta} .
$$

Write $D_{2}=F_{1}+F_{2}$, where

$$
\begin{aligned}
& F_{1}=\int_{B_{\rho}(\hat{x})} \eta_{\epsilon}(y) \int e^{i(x-y) \cdot \xi}(i \xi)^{\alpha}\left(\frac{1}{p(y, i \xi)}-\frac{1}{p(x, i \xi)}\right) \varphi\left(\frac{\xi}{R}\right) d \xi d y \\
& F_{2}=\int_{B_{\rho}(\hat{x})} \eta_{\epsilon}(y) \int e^{i(x-y) \cdot \xi}(i \xi)^{\alpha}\left(\frac{1}{p(x, i \xi)}-\frac{1}{p(\bar{x}, i \xi)}\right) \varphi\left(\frac{\xi}{R}\right) d \xi d y
\end{aligned}
$$

and we estimate

$$
\left|F_{1}\right| \leq C \int_{B_{\rho}(\hat{x})}|y-x|^{\delta} \frac{1}{|x-y|^{n}} d y \leq C \rho^{\delta} \leq C|x-\bar{x}|^{\delta}
$$

and

$$
\begin{aligned}
\left|F_{2}\right| & \leq \sum_{|\beta| \leq 2 m}\left|a_{\beta}(x)-a_{\beta}(\bar{x})\right|\left|\int_{B_{\rho}(\hat{x})} \eta_{\epsilon}(y) \int e^{i(x-y) \cdot \xi} \frac{(i \xi)^{\alpha}(i \xi)^{\beta}}{p(x, i \xi) p(\bar{x}, i \xi)} \varphi\left(\frac{\xi}{R}\right) d \xi d y\right| \\
& \leq C|x-\bar{x}|^{\delta},
\end{aligned}
$$

by Lemma 3.3 This finishes the estimation of $C_{1}$. Now,

$$
\left|C_{2}\right| \leq C \int_{B_{\rho}(\hat{x})} \frac{1}{|\bar{x}-y|^{n-\delta}} d y \leq C|x-\bar{x}|^{\delta}
$$

and

$$
\left|C_{3}\right| \leq C|x-\bar{x}| \int_{R^{n} \backslash B_{\rho}(\hat{x})} \frac{1}{|y-\hat{x}|^{n+1-\delta}} d y \leq C|x-\bar{x}|^{\delta} .
$$

This finishes the estimation of the term $B$. The lemma is proved.

We notice that

$$
L\left(N\left(g \eta_{\epsilon}\right)\right)(x)=\lim _{R \rightarrow \infty} \int g(y) \eta_{\epsilon}(y) \int e^{i(x-y) \cdot \xi} \frac{p(x, i \xi)}{p(y, i \xi)} \varphi\left(\frac{\xi}{R}\right) d \xi d y
$$

and also

$$
g \eta_{\epsilon}(x)=\lim _{R \rightarrow \infty} \int g(y) \eta_{\epsilon}(y) \int e^{i(x-y) \cdot \xi} \varphi\left(\frac{\xi}{R}\right) d \xi d y .
$$

Hence, we define

$$
\begin{aligned}
T\left(g \eta_{\epsilon}\right)(x) & :=L\left(N\left(g \eta_{\epsilon}\right)\right)(x)-g \eta_{\epsilon}(x) \\
& =\lim _{R \rightarrow \infty} \int g(y) \eta_{\epsilon}(y) \int e^{i(x-y) \cdot \xi} \frac{p(x, i \xi)-p(y, i \xi)}{p(y, i \xi)} \varphi\left(\frac{\xi}{R}\right) d \xi d y .
\end{aligned}
$$


Remark 3.6. If the coefficients $a_{\alpha}$ are constant then $u:=N\left(g \eta_{\epsilon}\right)$ solves $L u=g \eta_{\epsilon}$ in $\mathbb{R}^{n}$.

We can now state the main theorem of this section.

Theorem 3.7. There exists a constant $C$ depending on $K_{0}, \lambda$, and $n$ such that $\left|T\left(g \eta_{\epsilon}\right)\right|_{\delta ; B_{\epsilon}} \leq C K_{\delta, \epsilon}|g|_{\delta ; B_{\epsilon}}$.

Proof. For fixed $R \geq 1$, let

$$
I_{R}(x)=\int g(y) \eta_{\epsilon}(y) \int e^{i(x-y) \cdot \xi} \frac{p(x, i \xi)-p(y, i \xi)}{p(y, i \xi)} \varphi\left(\frac{\xi}{R}\right) d \xi d y .
$$

We write

$$
\begin{aligned}
I_{R}(x)-I_{R}(\bar{x})= & \int g(y) \eta_{\epsilon}(y) \int\left(e^{i(x-y) \cdot \xi}-e^{i(\bar{x}-y) \cdot \xi}\right) \frac{p(x, i \xi)-p(y, i \xi)}{p(y, i \xi)} \varphi\left(\frac{\xi}{R}\right) d \xi d y \\
& +\int g(y) \eta_{\epsilon}(y) \int e^{i(\bar{x}-y) \cdot \xi} \frac{p(x, i \xi)-p(\bar{x}, i \xi)}{p(y, i \xi)} \varphi\left(\frac{\xi}{R}\right) d \xi d y=A+B .
\end{aligned}
$$

Write $A=A_{1}+g(x) A_{2}$, where

$$
\begin{aligned}
& A_{1}=\int(g(y)-g(\bar{x})) \eta_{\epsilon}(y) \int\left(e^{i(x-y) \cdot \xi}-e^{i(\bar{x}-y) \cdot \xi}\right) \frac{p(x, i \xi)-p(y, i \xi)}{p(y, i \xi)} \varphi\left(\frac{\xi}{R}\right) d \xi d y, \\
& A_{2}=\int \eta_{\epsilon}(y) \int\left(e^{i(x-y) \cdot \xi}-e^{i(\bar{x}-y) \cdot \xi}\right) \frac{p(x, i \xi)-p(y, i \xi)}{p(y, i \xi)} \varphi\left(\frac{\xi}{R}\right) d \xi d y .
\end{aligned}
$$

Next, write $A_{1}=B_{1}-B_{2}+B_{3}$, where

$$
\begin{aligned}
B_{1} & =\int_{B_{\rho}(\hat{x})}(g(y)-g(\bar{x})) \eta_{\epsilon}(y) \int e^{i(x-y) \cdot \xi} \frac{p(x, i \xi)-p(y, i \xi)}{p(y, i \xi)} \varphi\left(\frac{\xi}{R}\right) d \xi d y, \\
B_{2}= & \int_{B_{\rho}(\hat{x})}(g(y)-g(\bar{x})) \eta_{\epsilon}(y) \int e^{i(\bar{x}-y) \cdot \xi} \frac{p(x, i \xi)-p(y, i \xi)}{p(y, i \xi)} \varphi\left(\frac{\xi}{R}\right) d \xi d y, \\
B_{3}=\int_{R^{n} \backslash B_{\rho}(\hat{x})}(g(y)-g(\bar{x})) \eta_{\epsilon}(y) \int\left(e^{i(x-y) \cdot \xi}-e^{i(\bar{x}-y) \cdot \xi}\right) & \times \frac{p(x, i \xi)-p(y, i \xi)}{p(y, i \xi)} \varphi\left(\frac{\xi}{R}\right) d \xi d y .
\end{aligned}
$$

We estimate, using Lemma 3.1 .

$$
\begin{aligned}
& \left|B_{1}\right| \leq K_{\delta ; \epsilon}[g] \int_{B_{\rho}(\hat{x})}|y-\bar{x}|^{\delta}|y-x|^{\delta} \frac{1}{|y-x|^{n}} d y \leq K_{\delta ; \epsilon}[g]|x-\bar{x}|^{\delta}, \\
& \left|B_{2}\right| \leq K_{\delta ; \epsilon}[g] \int_{B_{\rho}(\hat{x})}|y-\bar{x}|^{\delta}|y-x|^{\delta} \frac{1}{|y-\bar{x}|^{n}} d y \leq K_{\delta ; \epsilon}[g]|x-\bar{x}|^{\delta},
\end{aligned}
$$

and

$$
\begin{aligned}
\left|B_{3}\right| & \leq K_{\delta ; \epsilon}[g]|x-\bar{x}| \int_{0}^{1} \int_{R^{n} \backslash B_{\rho}(\hat{x})}|y-\bar{x}|^{\delta}|y-x|^{\delta} \frac{1}{\left|y-x_{s}\right|^{n+1}} d y d s \\
& \leq K_{\delta ; \epsilon}[g]|x-\bar{x}| \int_{R^{n} \backslash B_{\rho}(\hat{x})} \frac{1}{|y-\hat{x}|^{n+1-\delta}} d y \leq K_{\delta ; \epsilon}[g]|x-\bar{x}|^{\delta},
\end{aligned}
$$


finishing the estimation of $A_{1}$.

Write $A_{2}=C_{1}-C_{2}+C_{3}$, where

$$
\begin{aligned}
C_{1} & =\int_{B_{\rho}(\hat{x})} \eta_{\epsilon}(y) \int e^{i(x-y) \cdot \xi} \frac{p(x, i \xi)-p(y, i \xi)}{p(y, i \xi)} \varphi\left(\frac{\xi}{R}\right) d \xi d y, \\
C_{2} & =\int_{B_{\rho}(\hat{x})} \eta_{\epsilon}(y) \int e^{i(\bar{x}-y) \cdot \xi} \frac{p(x, i \xi)-p(y, i \xi)}{p(y, i \xi)} \varphi\left(\frac{\xi}{R}\right) d \xi d y, \\
C_{3} & =\int_{R^{n} \backslash B_{\rho}(\hat{x})} \eta_{\epsilon}(y) \int\left(e^{i(x-y) \cdot \xi}-e^{i(\bar{x}-y) \cdot \xi}\right) \frac{p(x, i \xi)-p(y, i \xi)}{p(y, i \xi)} \varphi\left(\frac{\xi}{R}\right) d \xi d y,
\end{aligned}
$$

and estimate by Lemma 3.1

$$
\left|C_{1}\right| \leq K_{\delta ; \epsilon} \int_{B_{\rho}(\hat{x})}|x-y|^{\delta} \frac{1}{|x-y|^{n}} d y \leq K_{\delta ; \epsilon}|x-\bar{x}|^{\delta} .
$$

Write $C_{2}=E_{1}+E_{2}$, where

$$
\begin{aligned}
& E_{1}=\int_{B_{\rho}(\hat{x})} \eta_{\epsilon}(y) \int e^{i(\bar{x}-y) \cdot \xi} \frac{p(x, i \xi)-p(\bar{x}, i \xi)}{p(\bar{x}, i \xi)} \varphi\left(\frac{\xi}{R}\right) d \xi d y \\
& E_{2}=\int_{B_{\rho}(\hat{x})} \eta_{\epsilon}(y) \int e^{i(\bar{x}-y) \cdot \xi} \frac{p(x, i \xi)(p(\bar{x}, i \xi)-p(y, i \xi))}{p(y, i \xi) p(\bar{x}, i \xi)} \varphi\left(\frac{\xi}{R}\right) d \xi d y,
\end{aligned}
$$

and estimate

$$
\begin{aligned}
\left|E_{1}\right| & \leq \sum_{|\alpha| \leq 2 m}\left|a_{\alpha}(x)-a_{\alpha}(\bar{x})\right|\left|\int_{B_{\rho}(\hat{x})} \eta_{\epsilon}(y) \int e^{i(\bar{x}-y) \cdot \xi} \frac{(i \xi)^{\alpha}}{p(\bar{x}, i \xi)} \varphi\left(\frac{\xi}{R}\right) d \xi d y\right| \\
& \leq K_{\delta ; \epsilon}|x-\bar{x}|^{\delta}
\end{aligned}
$$

by Lemma 3.3 and by Lemma 3.1 we have

$$
\left|E_{2}\right| \leq K_{\delta ; \epsilon} \int_{B_{\rho}(\hat{x})}|\bar{x}-y|^{\delta} \frac{1}{|\bar{x}-y|^{n}} d y \leq K_{\delta ; \epsilon}|x-\bar{x}|^{\delta} .
$$

We also have

$$
\left|C_{3}\right| \leq K_{\delta ; \epsilon}|x-\bar{x}| \int_{R^{n} \backslash B_{\rho}(\hat{x})} \int_{0}^{1} \frac{1}{\left|y-x_{s}\right|^{n+1}}|y-x|^{\delta} d s d y \leq K_{\delta ; \epsilon}|x-\bar{x}|^{\delta},
$$

thus finishing the estimation of $A_{2}$ and hence of $A$.

To estimate $B$ write $B=F_{1}+g(\bar{x}) F_{2}$, where

$$
\begin{aligned}
& F_{1}=\int(g(y)-g(\bar{x})) \eta_{\epsilon}(y) \int e^{i(\bar{x}-y) \cdot \xi} \frac{p(x, i \xi)-p(\bar{x}, i \xi)}{p(y, i \xi)} \varphi\left(\frac{\xi}{R}\right) d \xi d y, \\
& F_{2}=\int \eta_{\epsilon}(y) \int e^{i(\bar{x}-y) \cdot \xi} \frac{p(x, i \xi)-p(\bar{x}, i \xi)}{p(y, i \xi)} \varphi\left(\frac{\xi}{R}\right) d \xi d y
\end{aligned}
$$

and we have by Lemma 3.1

$$
\left|F_{1}\right| \leq K_{\delta ; \epsilon}[g]|x-\bar{x}|^{\delta} \int_{B_{\epsilon}}|y-\bar{x}|^{\delta} \frac{1}{|y-\bar{x}|^{n}} d y \leq K_{\delta ; \epsilon}[g]|x-\bar{x}|^{\delta} .
$$


Write $F_{2}=G_{1}+G_{2}$, where

$$
\begin{aligned}
G_{1} & =\int \eta_{\epsilon}(y) \int e^{i(\bar{x}-y) \cdot \xi} \frac{p(x, i \xi)-p(\bar{x}, i \xi)}{p(\bar{x}, i \xi)} \varphi\left(\frac{\xi}{R}\right) d \xi d y \\
G_{2} & =\int \eta_{\epsilon}(y) \int e^{i(\bar{x}-y) \cdot \xi}(p(x, i \xi)-p(\bar{x}, i \xi)) \frac{(p(\bar{x}, i \xi)-p(y, i \xi))}{p(\bar{x}, i \xi) p(y, i \xi)} \varphi\left(\frac{\xi}{R}\right) d \xi d y .
\end{aligned}
$$

We have

$$
\left|G_{1}\right| \leq \sum_{|\alpha| \leq 2 m}\left|a_{\alpha}(x)-a_{\alpha}(\bar{x})\right|\left|\int \eta_{\epsilon}(y) \int e^{i(\bar{x}-y) \cdot \xi} \frac{(i \xi)^{\alpha}}{p(\bar{x}, i \xi)} \varphi\left(\frac{\xi}{R}\right) d \xi d y\right| \leq K_{\delta ; \epsilon}|x-\bar{x}|^{\delta}
$$

by Lemma 3.2, and by Lemma 3.1 we have

$$
\left|G_{2}\right| \leq K_{\delta ; \epsilon}|x-\bar{x}|^{\delta} \int_{B_{\epsilon}}|y-\bar{x}|^{\delta} \frac{1}{|y-\bar{x}|^{n}} d y \leq K_{\delta ; \epsilon}|x-\bar{x}|^{\delta} .
$$

This finishes the estimation of the term $B$.

So we have shown that $\left[T\left(g \eta_{\epsilon}\right)\right]_{\delta ; B_{\epsilon}} \leq C K_{\delta, \epsilon}|g|_{\delta ; B_{\epsilon}}$.

The proof that $\left|T\left(g \eta_{\epsilon}\right)\right|_{0 ; B_{\epsilon}} \leq C K_{\delta ; \epsilon}|g|_{0 ; B_{\epsilon}}$ follows similarly and we omit it.

We are now ready to prove local existence of solutions of $L u=f$ when $f \in$ $C^{\delta}\left(B_{\epsilon}\right)$.

Theorem 3.8. Assume the operator L satisfies (2.3), 2.1) and (2.2). Then there exists $\epsilon>0$ depending on $K_{0}, \lambda$ and $n$ so that given $f \in C^{\delta}\left(\overrightarrow{B_{\epsilon}}\right)$, there exists $u \in C^{2 m+\delta}\left(B_{\epsilon}\right)$ such that $L u=f$ in $B_{\frac{\epsilon}{2}}$.

Proof. For $0<\epsilon$ to be chosen, we define a sequence of functions $g_{k}$ in $C^{\delta}\left(B_{\epsilon}\right)$ as follows:

Let $g_{0}=f$ and $g_{k+1}=f-T\left(g_{k} \eta_{\epsilon}\right)$.

By Lemma 3.5 we have $g_{k}$ in $C^{\delta}\left(B_{\epsilon}\right)$, and by Theorem 3.7 we have

$$
\begin{aligned}
\left|g_{k+1}-g_{k}\right|_{\delta ; B_{\epsilon}} & =\left|T\left(\eta_{\epsilon}\left(g_{k}-g_{k-1}\right)\right)\right|_{\delta ; B_{\epsilon}} \leq C K_{\delta ; \epsilon}\left|g_{k}-g_{k-1}\right|_{\delta ; B_{\epsilon}} \\
& \leq \epsilon^{\beta-\delta} K_{\epsilon, \beta}\left|g_{k}-g_{k-1}\right|_{\delta ; B_{\epsilon}} .
\end{aligned}
$$

Choosing $\epsilon$ small so that $\epsilon^{\beta-\delta} K_{\epsilon, \beta}<1$, by the contraction mapping theorem we can conclude that there exists $g \in C^{\delta}\left(B_{\epsilon}\right)$ such that $g=f-T\left(g \eta_{\epsilon}\right)$, which gives $g=f-L\left(N\left(g \eta_{\epsilon}\right)\right)+g \eta_{\epsilon}$. In particular, since $\eta_{\epsilon}=1$ in $B_{\frac{\epsilon}{2}}$, we have $L\left(N\left(g \eta_{\epsilon}\right)\right)=f$ in $B_{\frac{\epsilon}{2}}$. Let $u=N\left(g \eta_{\epsilon}\right)$. So $u \in C^{2 m+\delta}\left(B_{\epsilon}\right)$ and $L u=f$ in $B_{\frac{\epsilon}{2}}$.

\section{Local EXistence in half SPACE. Boundary CONDitions}

In this section we prove local existence of solutions in half space.

We will show that there exists $\epsilon>0$ such that given $f \in C^{\delta}\left(B_{\epsilon}^{+}\right)$there exists $u \in C^{2 m+\delta}\left(B_{\epsilon}^{+}\right)$such that $L u=f$ in $B_{\epsilon}^{+}$and such that $u$ satisfies the $m$ boundary conditions,

$$
\frac{\partial^{k} u\left(x^{\prime}, 0\right)}{\partial x_{n}^{k}}=0
$$

for $k=0, \ldots, m-1$. 
Given $g \in C^{\delta}\left(B_{\epsilon}\right)$, we define the approximate solution $N^{+}\left(\eta_{\epsilon} g\right)$ by the following formula. For $x \in B_{\epsilon}^{+}$let

$$
N^{+}\left(\eta_{\epsilon} g\right)(x)=\lim _{R \rightarrow \infty} \int_{R_{+}^{n}} g(y) \eta_{\epsilon}(y) \int \varphi\left(\frac{\xi}{R}\right) \frac{e^{i(x-y) \cdot \xi}}{p(y, i \xi)} d \xi d y .
$$

In order to achieve the boundary conditions, we define for $x \in B_{\epsilon}^{+}$

$$
H\left(\eta_{\epsilon} g\right)(x)=\int_{R_{+}^{n}} g(y) \eta_{\epsilon}(y) \int e^{i\left(x^{\prime}-y^{\prime}\right) \cdot \xi^{\prime}} \Phi\left(y, x, \xi^{\prime}\right) d \xi^{\prime} d y
$$

where $\Phi\left(y, x, \xi^{\prime}\right)$ is given by

$$
\Phi\left(y, x, \xi^{\prime}\right)=\frac{1}{2 \pi i} \int_{\gamma^{+}\left(\xi^{\prime}\right)} \int_{\gamma^{-}\left(\xi^{\prime}\right)} \frac{e^{-y_{n} z} e^{x_{n} w}}{a(y) p^{+}\left(y, i \xi^{\prime}, z\right) p^{-}\left(y, i \xi^{\prime}, w\right)(w-z)} d w d z,
$$

where $\gamma^{+}\left(\xi^{\prime}\right)$ denotes any piecewise smooth contour on the right half plane enclosing the roots of $p^{+}\left(y, i \xi^{\prime}, z\right)$. And $\gamma^{-}\left(\xi^{\prime}\right)$ denotes any piecewise smooth contour on the left half plane enclosing the roots of $p^{-}\left(y, i \xi^{\prime}, z\right)$.

Defining the function

$$
u:=N^{+}\left(\eta_{\epsilon} g\right)-H\left(\eta_{\epsilon} g\right)
$$

we will show in this section that

$$
\begin{aligned}
L u(x)= & \lim _{R \rightarrow \infty} \int_{R_{+}^{n}} g(y) \eta_{\epsilon}(y) \int \varphi\left(\frac{\xi}{R}\right) \frac{e^{i(x-y) \cdot \xi} p(x, i \xi)}{p(y, i \xi)} d \xi d y \\
& -\int_{R_{+}^{n}} g(y) \eta_{\epsilon}(y) \int e^{i\left(x^{\prime}-y^{\prime}\right) \cdot \xi^{\prime}} \frac{1}{2 \pi i} \int_{\gamma^{+}\left(\xi^{\prime}\right)} \int_{\gamma^{-}\left(\xi^{\prime}\right)} \\
& \frac{e^{-y_{n} z} e^{x_{n} w}\left(p\left(x, i \xi^{\prime}, w\right)-p\left(y, i \xi^{\prime}, w\right)\right)}{a(y) p^{+}\left(y, i \xi^{\prime}, z\right) p^{-}\left(y, i \xi^{\prime}, w\right)(w-z)} d w d z .
\end{aligned}
$$

In particular, for constant coefficient operators, we have $L u=\eta_{\epsilon} g$.

We will also show in this section that

$$
\frac{\partial^{k} u\left(x^{\prime}, 0\right)}{\partial x_{n}^{k}}=0
$$

for $k=0, \ldots, m-1$.

4.1. Auxiliary integrals in half space for the operator $N^{+}$. We proceed to prove estimates for the auxiliary integrals we need in half space related to the operator $\mathrm{N}^{+}$.

For $x \in \mathbb{R}^{n}$, we write $x=\left(x^{\prime}, x_{n}\right)$ with $x^{\prime} \in \mathbb{R}^{n-1}$.

Lemma 4.1. Let

$$
A_{R}\left(x, \xi^{\prime}\right)=\int \frac{e^{i x_{n} \xi_{n}} \xi_{n}^{2 m-1}}{p(x, i \xi)} \varphi\left(\frac{\xi^{\prime}}{R}, \frac{\xi_{n}}{R}\right) d \xi_{n} .
$$

We have $\left|A_{R}\left(x, \xi^{\prime}\right)\right| \leq C$, for $x \in \mathbb{R}_{+}^{n}$ and $\xi^{\prime} \in \mathbb{R}^{n-1}$. The constant $C$ depends only on $K_{0}, \lambda$ and $n$. 
Proof. Write

$$
p(x, i \xi)=a(x)\left(i \xi_{n}\right)^{2 m}+\sum_{k=1}^{2 m}\left(i \xi_{n}\right)^{2 m-k} \sum_{|\alpha| \leq k} a_{\alpha}(x)\left(i \xi^{\prime}\right)^{\alpha} .
$$

Here $\alpha$ is an $n-1$ multi-index.

A change of variables $s=x_{n} \xi_{n}, d s=x_{n} d \xi_{n}$, gives

$$
A_{R}=\int \frac{e^{i s} s^{2 m-1}}{x_{n}^{2 m} p\left(x, \xi^{\prime}, \frac{s}{x_{n}}\right)} \varphi\left(\frac{\xi^{\prime}}{R}, \frac{s}{x_{n} R}\right) d s
$$

Note that

$x_{n}^{2 m} p\left(x, i \xi^{\prime}, \frac{i s}{x_{n}}\right)=a(x)(i s)^{2 m}+\sum_{k=1}^{2 m} x_{n}^{k}(i s)^{2 m-k} \sum_{|\alpha| \leq k} a_{\alpha}(x)\left(i \xi^{\prime}\right)^{\alpha}=a(x)(i s)^{2 m}+q$

and

$$
\left|x_{n}^{2 m} p\left(x, i \xi^{\prime}, \frac{i s}{x_{n}}\right)\right| \geq C\left(x_{n}^{2 m}+x_{n}^{2 m}\left|\xi^{\prime}\right|^{2 m}+s^{2 m}\right) .
$$

We write $\left|a(x)(i s)^{2 m}+q\right|^{2}=|a(x)|^{2} s^{4 m}+Q$, where $Q=Q\left(x, s, \xi^{\prime}\right)$, and note that

$$
|a(x)|^{2} s^{4 m}+Q \geq C\left(x_{n}^{4 m}+x_{n}^{4 m}\left|\xi^{\prime}\right|^{4 m}+s^{4 m}\right)
$$

and

$$
|Q| \leq C s^{2 m} \sum_{k=1}^{2 m} x_{n}^{k} s^{2 m-k}\left(1+\left|\xi^{\prime}\right|\right)^{k}+C \sum_{k=1}^{2 m} x_{n}^{2 k} s^{4 m-2 k}\left(1+\left|\xi^{\prime}\right|\right)^{2 k} .
$$

Hence,

$$
\begin{aligned}
A_{R} & =\bar{a}(x) \int \frac{e^{i s} s^{4 m-1}}{|a(x)|^{2} s^{4 m}+Q} \varphi\left(\frac{\xi^{\prime}}{R}, \frac{s}{x_{n} R}\right) d s+\int \frac{e^{i s} s^{2 m-1} \bar{q}}{|a(x)|^{2} s^{4 m}+Q} \varphi\left(\frac{\xi^{\prime}}{R}, \frac{s}{x_{n} R}\right) d s \\
& =\bar{a}(x) A+B .
\end{aligned}
$$

Write

$$
\begin{aligned}
A & =\int \frac{\cos (s) s^{4 m-1}}{|a(x)|^{2} s^{4 m}+Q} \varphi\left(\frac{\xi^{\prime}}{R}, \frac{s}{x_{n} R}\right) d s+i \int \frac{\sin (s) s^{4 m-1}}{|a(x)|^{2} s^{4 m}+Q} \varphi\left(\frac{\xi^{\prime}}{R}, \frac{s}{x_{n} R}\right) d s \\
& =A_{1}+A_{2} .
\end{aligned}
$$

Notice that

$$
\begin{aligned}
A_{1} & =\int_{-2 x_{n} R}^{2 x_{n} R} \frac{\cos (s) s^{4 m-1}}{|a(x)|^{2} s^{4 m}+Q} \varphi\left(\frac{\xi^{\prime}}{R}, \frac{s}{x_{n} R}\right) d s \\
& =\int_{0}^{2 x_{n} R} \cos (s) s^{4 m-1} \varphi\left(\frac{\xi^{\prime}}{R}, \frac{s}{x_{n} R}\right)\left(\frac{1}{|a(x)|^{2} s^{4 m}+Q(s)}-\frac{1}{|a(x)|^{2} s^{4 m}+Q(-s)}\right) d s \\
& =\int_{0}^{2 x_{n} R} \cos (s) s^{4 m-1} \varphi\left(\frac{\xi^{\prime}}{R}, \frac{s}{x_{n} R}\right) \frac{Q(s)-Q(-s)}{\left(|a(x)|^{2} s^{4 m}+Q(s)\right)\left(|a(x)|^{2} s^{4 m}+Q(-s)\right)} d s .
\end{aligned}
$$


Hence, we have

$$
\begin{aligned}
\left|A_{1}\right| & \leq \int_{0}^{\infty} s^{4 m-1} \frac{|Q(s)|+|Q(-s)|}{s^{8 m}+x_{n}^{8 m}\left(1+\left|\xi^{\prime}\right|\right)^{8 m}} d s \\
& \leq \sum_{k=1}^{2 m} \int_{0}^{\infty} \frac{s^{8 m-k-1} x_{n}^{k}\left(1+\left|\xi^{\prime}\right|\right)^{k}}{s^{8 m}+x_{n}^{8 m}\left(1+\left|\xi^{\prime}\right|\right)^{8 m}} d s+\sum_{k=1}^{2 m} \int_{0}^{\infty} \frac{s^{8 m-2 k-1} x_{n}^{2 k}\left(1+\left|\xi^{\prime}\right|\right)^{2 k}}{s^{8 m}+x_{n}^{8 m}\left(1+\left|\xi^{\prime}\right|\right)^{8 m}} d s \\
& \leq c \sum_{k=1}^{2 m} \int_{0}^{\infty} \frac{t^{8 m-2 k-1}}{1+t^{8 m}} d s \leq C
\end{aligned}
$$

and

$$
\begin{aligned}
A_{2} & =\int_{-1}^{1} \frac{\sin (s) s^{4 m-1}}{|a(x)|^{2} s^{4 m}+Q} \varphi\left(\frac{\xi^{\prime}}{R}, \frac{s}{x_{n} R}\right) d s+\int_{|s| \geq 1} \frac{\sin (s) s^{4 m-1}}{|a(x)|^{2} s^{4 m}+Q} \varphi\left(\frac{\xi^{\prime}}{R}, \frac{s}{x_{n} R}\right) d s \\
& =B_{1}+B_{2} .
\end{aligned}
$$

We have $\left|B_{1}\right| \leq 1$. For $B_{2}$, we assume $2 x_{n} R \geq 1$, since otherwise $B_{2}=0$. Write

$$
\begin{aligned}
B_{2} & =\int_{1}^{+\infty} \frac{\sin (s) s^{4 m-1}}{|a(x)|^{2} s^{4 m}+Q} \varphi\left(\frac{\xi^{\prime}}{R}, \frac{s}{x_{n} R}\right) d s+\int_{-\infty}^{-1} \frac{\sin (s) s^{4 m-1}}{|a(x)|^{2} s^{4 m}+Q} \varphi\left(\frac{\xi^{\prime}}{R}, \frac{s}{x_{n} R}\right) d s \\
& =C_{1}+C_{2}
\end{aligned}
$$

We have that

$$
C_{1}=\frac{\cos (1)}{|a(x)|^{2} s^{4 m}+Q(1)}+\int_{1}^{+\infty} \cos (s) \frac{d}{d s}\left(\frac{s^{4 m-1} \varphi\left(\frac{\xi^{\prime}}{R}, \frac{s}{x_{n} R}\right)}{|a(x)|^{2} s^{4 m}+Q(s)}\right) d s .
$$

It follows easily that $\left|C_{1}\right| \leq C$, and analogously we obtain $\left|C_{2}\right| \leq C$. Also,

$$
B=\sum_{k=1}^{2 m} \sum_{|\alpha| \leq k} \bar{a}_{\alpha}(x)\left(\xi^{\prime}\right)^{\alpha} x_{n}^{k} \int \frac{e^{i s} s^{4 m-1-k}}{|a(x)|^{2} s^{4 m}+Q(s)} \varphi\left(\frac{\xi^{\prime}}{R}, \frac{s}{x_{n} R}\right) d s .
$$

Change variables, $t=\frac{s}{x_{n}\left(1+\left|\xi^{\prime}\right|\right)}$, to get

$$
\begin{aligned}
B= & \sum_{k=1}^{2 m} \sum_{|\alpha| \leq k} \bar{a}_{\alpha}(x)\left(\xi^{\prime}\right)^{\alpha} x_{n}^{k}\left(x_{n}\left(1+\left|\xi^{\prime}\right|\right)\right)^{4 m-k} \\
& \times \int \frac{e^{i t x_{n}\left(1+\left|\xi^{\prime}\right|\right)} t^{4 m-1-k} \varphi\left(\frac{\xi^{\prime}}{R}, \frac{t\left(1+\left|\xi^{\prime}\right|\right)}{R}\right) d t}{|a(x)|^{2} t^{4 m} x_{n}^{4 m}\left(1+\left|\xi^{\prime}\right|\right)^{4 m}+Q\left(t x_{n}\left(1+\left|\xi^{\prime}\right|\right)\right)} \\
= & \sum_{k=1}^{2 m} \sum_{|\alpha| \leq k} \bar{a}_{\alpha}(x) \frac{\left(\xi^{\prime}\right)^{\alpha}}{\left(1+\left|\xi^{\prime}\right|\right)^{k}} \int \frac{e^{i t x_{n}\left(1+\left|\xi^{\prime}\right|\right)} t^{4 m-1-k} \varphi\left(\frac{\xi^{\prime}}{R}, \frac{t\left(1+\left|\xi^{\prime}\right|\right)}{R}\right) d t}{|a(x)|^{2} t^{4 m}+Q\left(t x_{n}\left(1+\left|\xi^{\prime}\right|\right)\right)\left(x_{n}\left(1+\left|\xi^{\prime}\right|\right)\right)^{-4 m}} .
\end{aligned}
$$

Note that $|a(x)|^{2} t^{4 m}+Q\left(t x_{n}\left(1+\left|\xi^{\prime}\right|\right)\right)\left(x_{n}\left(1+\left|\xi^{\prime}\right|\right)\right)^{-4 m} \geq C\left(1+t^{4 m}\right)$. Hence,

$$
|B| \leq \sum_{k=1}^{2 m} \sum_{|\alpha| \leq k}\left|a_{\alpha}(x)\right| \int_{-\infty}^{+\infty} \frac{|t|^{4 m-k-1}}{1+t^{4 m}} d t \leq C
$$


finishing the proof of the lemma.

Lemma 4.2. Let

$$
I_{R}=\int \frac{e^{i\left(x^{\prime}-y^{\prime}\right) \cdot \xi^{\prime}}}{1+\left|\xi^{\prime}\right|^{2}} \int \frac{e^{i x_{n} \xi_{n}} \xi_{n}^{2 m-1}}{p(x, \xi)} \varphi\left(\frac{\xi}{R}\right) d \xi_{n} d \xi^{\prime} .
$$

We have $\left|I_{R}\right| \leq \frac{C}{\left|x^{\prime}-y^{\prime}\right|^{n-3}}$, with the constant $C$ depending only on $K_{0}, \lambda$ and $n$.

Proof. Write

$$
\begin{aligned}
I_{R}= & \frac{1}{\left|x^{\prime}-y^{\prime}\right|^{2 k}} \int\left(-\Delta_{\xi^{\prime}}\right)^{k} e^{i\left(x^{\prime}-y^{\prime}\right) \cdot \xi^{\prime}} \int \frac{e^{i x_{n} \xi_{n}} \xi_{n}^{2 m-1}}{\left(1+\left|\xi^{\prime}\right|^{2}\right) p(x, i \xi)} \varphi\left(\frac{\xi}{R}\right) d \xi_{n} d \xi^{\prime} \\
= & \frac{1}{\left|x^{\prime}-y^{\prime}\right|^{2 k}} \int e^{i\left(x^{\prime}-y^{\prime}\right) \cdot \xi^{\prime}} \int e^{i x_{n} \xi_{n}}\left(-\Delta_{\xi^{\prime}}\right)^{k}\left(\frac{\xi_{n}^{2 m-1} \varphi\left(\frac{\xi}{R}\right)}{\left(1+\left|\xi^{\prime}\right|^{2}\right) p(x, \xi)}\right) d \xi_{n} d \xi^{\prime} \\
= & \frac{1}{\left|x^{\prime}-y^{\prime}\right|^{2 k}} \int e^{i\left(x^{\prime}-y^{\prime}\right) \cdot \xi^{\prime}} \int e^{i x_{n} \xi_{n}}\left(-\Delta_{\xi^{\prime}}\right)^{k}\left(\frac{\xi_{n}^{2 m-1} \varphi\left(\frac{\xi}{R}\right)}{\left(1+\left|\xi^{\prime}\right|^{2}\right) p(x, i \xi)}\right) \\
& +\frac{1}{\left|x^{\prime}-y^{\prime}\right|^{2 k}} \int e^{i\left(x^{\prime}-y^{\prime}\right) \cdot \xi^{\prime}} \int e^{i x_{n} \xi_{n}}\left(-\Delta_{\xi^{\prime}}\right)^{k}\left(\begin{array}{c}
\times\left(\left|x^{\prime}-y^{\prime}\right| \xi^{\prime}\right) d \xi_{n} d \xi^{\prime} \\
\left(1+\left|\xi^{\prime}\right|^{2}\right) p(x, \xi)
\end{array}\right) \\
= & A+B .
\end{aligned}
$$

Rewrite

$$
\begin{aligned}
A=\frac{1}{\left|x^{\prime}-y^{\prime}\right|^{2 k}} \int_{\left|\xi^{\prime}\right| \leq \frac{2}{\left|x^{\prime}-y^{\prime}\right|}}\left(-\Delta_{\xi^{\prime}}\right)^{k}\left(e^{i\left(x^{\prime}-y^{\prime}\right) \cdot \xi^{\prime}} \varphi\left(\left|x^{\prime}-y^{\prime}\right| \xi^{\prime}\right)\right) \\
\\
\times \int \frac{e^{i x_{n} \xi_{n}} \xi_{n}^{2 m-1} \varphi\left(\frac{\xi}{R}\right)}{\left(1+\left|\xi^{\prime}\right|^{2}\right) p(x, i \xi)} d \xi_{n} d \xi^{\prime},
\end{aligned}
$$

and estimate

$$
\begin{aligned}
|A| & \leq \int_{\left|\xi^{\prime}\right| \leq \frac{2}{\left|x^{\prime}-y^{\prime}\right|}}\left|\int \frac{e^{i x_{n} \xi_{n}} \xi_{n}^{2 m-1} \varphi\left(\frac{\xi}{R}\right)}{p(x, i \xi)} d \xi_{n}\right| \frac{1}{1+\left|\xi^{\prime}\right|^{2}} d \xi^{\prime} \\
& \leq \int_{\left|\xi^{\prime}\right| \leq \frac{2}{\left|x^{\prime}-y^{\prime}\right|}} \frac{1}{1+\left|\xi^{\prime}\right|^{2}} d \xi^{\prime} \leq \frac{C}{\left|x^{\prime}-y^{\prime}\right|^{n-3}},
\end{aligned}
$$

by Lemma 4.1 .

To estimate $B$, first we claim that

$$
\left|\left(-\Delta_{\xi^{\prime}}\right)^{k}\left(\int \frac{e^{i x_{n} \xi_{n}} \xi_{n}^{2 m-1} \varphi\left(\frac{\xi}{R}\right)}{p(x, i \xi)} d \xi_{n} \frac{1}{1+\left|\xi^{\prime}\right|^{2}}\right)\right| \leq \frac{C}{\left(1+\left|\xi^{\prime}\right|\right)^{2+2 k}} .
$$


To prove the claim, write

$$
\begin{aligned}
\mid\left(-\Delta_{\xi^{\prime}}\right)^{k} & \left(\int \frac{e^{i x_{n} \xi_{n}} \xi_{n}^{2 m-1} \varphi\left(\frac{\xi}{R}\right)}{p(x, i \xi)} d \xi_{n} \frac{1}{1+\left|\xi^{\prime}\right|^{2}}\right) \mid \\
= & \int \frac{e^{i x_{n} \xi_{n}} \xi_{n}^{2 m-1} \varphi\left(\frac{\xi}{R}\right)}{p(x, i \xi)} d \xi_{n}\left(-\Delta_{\xi^{\prime}}\right)^{k}\left(\frac{1}{1+\left|\xi^{\prime}\right|^{2}}\right) \\
& +\sum_{|\gamma|+|\beta|=2 k,|\beta| \geq 1} D_{\xi^{\prime}}^{\beta}\left(\int \frac{e^{i x_{n} \xi_{n}} \xi_{n}^{2 m-1} \varphi\left(\frac{\xi}{R}\right)}{p(x, i \xi)} d \xi_{n}\right) D_{\xi^{\prime}}^{\gamma} \frac{1}{1+\left|\xi^{\prime}\right|^{2}} \\
= & B_{1}+B_{2} .
\end{aligned}
$$

We have, using Lemma 4.1 that

$$
\left|B_{1}\right| \leq C \frac{1}{\left(1+\left|\xi^{\prime}\right|\right)^{2+2 k}} .
$$

To bound $B_{2}$, note that $\left|D_{\xi^{\prime}}^{\gamma} \frac{1}{1+\left|\xi^{\prime}\right|^{2}}\right| \leq C \frac{1}{\left(1+\left|\xi^{\prime}\right|\right)^{2+|\gamma|}}$, and for $\left|\beta_{1}\right|+\left|\beta_{2}\right|=|\beta|$ we have

$$
\begin{aligned}
\mid \int e^{i x_{n} \xi_{n}} \xi_{n}^{2 m-1} & D^{\beta_{1}} \varphi\left(\frac{\xi}{R}\right) D^{\beta_{2}} \frac{1}{p(x, i \xi)} d \xi_{n} \mid \\
& \leq C X_{\left|\xi^{\prime}\right| \leq 2 R} \frac{1}{R^{\left|\beta_{1}\right|}} \int_{0}^{\infty} \xi_{n}^{2 m-1} \frac{1}{\left(1+\left|\xi^{\prime}\right|+\xi_{n}\right)^{2 m+\left|\beta_{2}\right|}} d \xi_{n} \\
& \leq C X_{\left|\xi^{\prime}\right| \leq 2 R} \frac{1}{R^{\left|\beta_{1}\right|}} \frac{1}{\left(1+\left|\xi^{\prime}\right|\right)^{\left|\beta_{2}\right|}} \\
& \leq C \frac{1}{\left(1+\left|\xi^{\prime}\right|\right)^{|\beta|}},
\end{aligned}
$$

proving the claim.

And hence we have

$$
|B| \leq \frac{C}{\left|x^{\prime}-y^{\prime}\right|^{2 k}} \int_{\left|\xi^{\prime}\right| \geq \frac{1}{\left|x^{\prime}-y^{\prime}\right|}} \frac{1}{\left(1+\left|\xi^{\prime}\right|\right)^{2+2 k}} d \xi^{\prime} \leq \frac{C}{\left|x^{\prime}-y^{\prime}\right|^{n-3}},
$$

finishing the proof of the lemma.

We use the estimate above to prove estimates for two auxiliary integrals in half space.

\section{Lemma 4.3. Define}

$$
I_{R}=\int_{R_{+}^{n}} \eta_{\epsilon}(y) \int \frac{e^{i(x-y) \cdot \xi} \xi_{n}^{2 m} \varphi\left(\frac{\xi}{R}\right)}{p(x, i \xi)} d \xi d y .
$$

We have $\left|I_{R}\right| \leq C$, with $C$ depending only on $K_{0}, \lambda$ and $n$. 
Proof. Write

$$
\begin{aligned}
I_{R}= & \int_{R_{+}^{n}} \eta_{\epsilon}(y) \frac{\partial}{\partial y_{n}}\left(\int \frac{e^{i(x-y) \cdot \xi} \xi_{n}^{2 m-1} \varphi\left(\frac{\xi}{R}\right)}{p(x, i \xi)} d \xi\right) d y \\
= & -\int_{R_{+}^{n}} \frac{\partial}{\partial y_{n}}\left(\eta_{\epsilon}(y)\right) \int \frac{e^{i(x-y) \cdot \xi} \xi_{n}^{2 m-1} \varphi\left(\frac{\xi}{R}\right)}{p(x, i \xi)} d \xi d y \\
& +\int_{R^{n-1}} \eta_{\epsilon}\left(y^{\prime}, 0\right) \int \frac{e^{i\left(x^{\prime}-y^{\prime}\right) \cdot \xi^{\prime}} e^{i x_{n} \xi_{n}} \xi_{n}^{2 m-1} \varphi\left(\frac{\xi}{R}\right)}{p(x, i \xi)} d \xi d y^{\prime} \\
= & A_{R}+B_{R} .
\end{aligned}
$$

We have

$$
\left|A_{R}\right| \leq \frac{C}{\epsilon} \int_{B_{\epsilon}^{+}} \frac{1}{|x-y|^{n-1}} d y \leq C
$$

and

$$
\begin{aligned}
B_{R} & =\int_{R^{n-1}} \eta_{\epsilon}\left(y^{\prime}, 0\right)\left(1-\Delta_{y^{\prime}}\right)\left(\int \frac{e^{i\left(x^{\prime}-y^{\prime}\right) \cdot \xi^{\prime}} e^{i x_{n} \xi_{n}} \xi_{n}^{2 m-1} \varphi\left(\frac{\xi}{R}\right)}{\left(1+\left|\xi^{\prime}\right|^{2}\right) p(x, i \xi)} d \xi\right) d y^{\prime} \\
& =\int_{R^{n-1}}\left(1-\Delta_{y^{\prime}}\right) \eta_{\epsilon}\left(y^{\prime}, 0\right) \int \frac{e^{i\left(x^{\prime}-y^{\prime}\right) \cdot \xi^{\prime}} e^{i x_{n} \xi_{n}} \xi_{n}^{2 m-1} \varphi\left(\frac{\xi}{R}\right)}{\left(1+\left|\xi^{\prime}\right|^{2}\right) p(x, i \xi)} d \xi d y^{\prime}
\end{aligned}
$$

hence

$$
\left|B_{R}\right| \leq \frac{C}{\epsilon^{2}} \int_{B_{\epsilon}^{\prime}} \frac{1}{\left|x^{\prime}-y^{\prime}\right|^{n-3}} d y^{\prime} \leq C .
$$

In the first inequality of the line above we have used Lemma 4.2 This proves the lemma.

We now consider a similar integral over a ball.

Lemma 4.4. Let $x, \bar{x} \in \mathbb{R}_{+}^{n}, \rho=2|x-\bar{x}|, \hat{x}=\frac{x+\bar{x}}{2}$, and $B_{\rho}^{+}(\hat{x})=B_{\rho}(\hat{x}) \cap \mathbb{R}_{+}^{n}$. Let

$$
I_{R}=\int_{B_{\rho}^{+}(\hat{x})} \eta_{\epsilon}(y) \int \frac{e^{i(x-y) \cdot \xi} \xi_{n}^{2 m} \varphi\left(\frac{\xi}{R}\right)}{p(x, i \xi)} d \xi d y
$$

Then we have $\left|I_{R}\right| \leq C$. 
Proof. Write

$$
\begin{aligned}
I_{R}= & \int_{B_{\rho}^{+}(\hat{x})} \eta_{\epsilon}(y) \frac{\partial}{\partial y_{n}}\left(\int \frac{e^{i(x-y) \cdot \xi} \xi_{n}^{2 m-1} \varphi\left(\frac{\xi}{R}\right)}{p(x, i \xi)} d \xi\right) d y \\
= & -\int_{B_{\rho}^{+}(\hat{x})} \frac{\partial}{\partial y_{n}}\left(\eta_{\epsilon}(y)\right) \int \frac{e^{i(x-y) \cdot \xi} \xi_{n}^{2 m-1} \varphi\left(\frac{\xi}{R}\right)}{p(x, i \xi)} d \xi d y \\
& +\int_{\partial B_{\rho}^{+}(\hat{x})} \eta_{\epsilon}(y) \int \frac{e^{i\left(x^{\prime}-y^{\prime}\right) \cdot \xi^{\prime}} e^{i x_{n} \xi_{n}} \xi_{n}^{2 m-1} \varphi\left(\frac{\xi}{R}\right)}{p(x, i \xi)} d \xi d S_{y} \\
= & A_{R}+B_{R} .
\end{aligned}
$$

We have

$$
\left|A_{R}\right| \leq \frac{C}{\epsilon} \int_{B_{\epsilon}} \frac{1}{|x-y|^{n-1}} d y \leq C
$$

and

$$
\begin{aligned}
B_{R}= & \int_{\partial B_{\rho}^{+}(\hat{x}) \cap R_{+}^{n}} \eta_{\epsilon}(y) \int \frac{e^{i\left(x^{\prime}-y^{\prime}\right) \cdot \xi^{\prime}} e^{i x_{n} \xi_{n}} \xi_{n}^{2 m-1} \varphi\left(\frac{\xi}{R}\right)}{p(x, i \xi)} d \xi d S_{y} \\
& +\int_{B_{\rho}^{+}(\hat{x}) \cap\left\{y_{n}=0\right\}} \eta_{\epsilon}(y) \int \frac{e^{i\left(x^{\prime}-y^{\prime}\right) \cdot \xi^{\prime}} e^{i x_{n} \xi_{n}} \xi_{n}^{2 m-1} \varphi\left(\frac{\xi}{R}\right)}{p(x, i \xi)} d \xi d y^{\prime}=C_{R}+D_{R}
\end{aligned}
$$

We can estimate

$$
\left|C_{R}\right| \leq \int_{\partial B_{\rho}^{+}(\hat{x}) \cap R_{+}^{n}} \frac{1}{|x-y|^{n-1}} d S_{y} \leq C .
$$

To estimate $D_{R}$, let $B_{\rho}^{+}(\hat{x}) \cap\left\{y_{n}=0\right\}=B_{r}^{\prime}\left(\hat{x}^{\prime}\right)$. Write

$$
\begin{aligned}
D_{R}= & \int_{B_{r}^{\prime}\left(\hat{x}^{\prime}\right)} \eta_{\epsilon}\left(y^{\prime}, 0\right) \int \frac{e^{i\left(x^{\prime}-y^{\prime}\right) \cdot \xi^{\prime}} e^{i x_{n} \xi_{n}} \xi_{n}^{2 m-1} \varphi\left(\frac{\xi}{R}\right)}{p(x, i \xi)} d \xi d y^{\prime} \\
= & \int_{B_{r}^{\prime}\left(\hat{x}^{\prime}\right)}\left(\eta_{\epsilon}\left(y^{\prime}, 0\right)-\eta_{\epsilon}\left(x^{\prime}, 0\right)\right) \int \frac{e^{i\left(x^{\prime}-y^{\prime}\right) \cdot \xi^{\prime}} e^{i x_{n} \xi_{n}} \xi_{n}^{2 m-1} \varphi\left(\frac{\xi}{R}\right)}{p(x, i \xi)} d \xi d y^{\prime} \\
& +\eta_{\epsilon}\left(x^{\prime}, 0\right) \int_{B_{r}^{\prime}\left(\hat{x}^{\prime}\right)} \int \frac{e^{i\left(x^{\prime}-y^{\prime}\right) \cdot \xi^{\prime}} e^{i x_{n} \xi_{n}} \xi_{n}^{2 m-1} \varphi\left(\frac{\xi}{R}\right)}{p(x, i \xi)} d \xi d y^{\prime}=E_{R}+\eta_{\epsilon}\left(x^{\prime}, 0\right) F_{R} .
\end{aligned}
$$

We can estimate

$$
\left|E_{R}\right| \leq \frac{C}{\epsilon} \int_{B_{r}^{\prime}\left(\hat{x}^{\prime}\right)}\left|x^{\prime}-y^{\prime}\right|^{2-n} d y^{\prime} \leq C,
$$

since $r \leq \rho \leq 2 \epsilon$.

To estimate $F_{R}$, consider first the case $\hat{x}_{n} \geq \frac{\rho}{2}$. Note that $\hat{x}_{n}-x_{n} \leq\left|\hat{x}_{n}-x_{n}\right| \leq$ $|x-\hat{x}|=\frac{\rho}{4}$. Therefore $x_{n} \geq \hat{x}_{n}-\frac{\rho}{4} \geq \frac{\rho}{4}$. 
In this case,

$$
\begin{aligned}
\left|F_{R}\right| & \leq \int_{B_{r}^{\prime}\left(\hat{x}^{\prime}\right)} \frac{1}{\left(\left|x^{\prime}-y^{\prime}\right|^{2}+x_{n}^{2}\right)^{\frac{n-1}{2}}} d y^{\prime} \leq \frac{1}{x_{n}^{n-1}} \int_{B_{r}^{\prime}\left(\hat{x}^{\prime}\right)} 1 d y^{\prime} \\
& \leq C\left(\frac{r}{x_{n}}\right)^{n-1} \leq C\left(\frac{\rho}{x_{n}}\right)^{n-1} \leq C .
\end{aligned}
$$

For the case $\hat{x}_{n} \leq \frac{\rho}{2}$, note that since $r^{2}+x_{n}^{2}=\rho^{2}$, we get $r^{2} \geq \rho^{2}-\frac{\rho^{2}}{4}=\frac{3}{4} \rho^{2}$, so $r \geq \frac{\sqrt{3}}{2} \rho$ and $\left|x^{\prime}-\hat{x}^{\prime}\right| \leq|x-\hat{x}|=\frac{\rho}{4} \leq \frac{1}{2 \sqrt{3}} r$ and hence, $\operatorname{dist}\left(x^{\prime}, \partial B_{r}^{\prime}\left(\hat{x}^{\prime}\right)\right) \geq$ $\left(1-\frac{1}{2 \sqrt{3}}\right) r$.

Hence, in this case we write

$$
\begin{aligned}
F_{R}= & \int_{B_{r}^{\prime}\left(\hat{x}^{\prime}\right)}\left(1-\Delta_{y^{\prime}}\right) \int \frac{e^{i\left(\left(x^{\prime}-y^{\prime}\right) \cdot \xi^{\prime}\right.} e^{i x_{n} \xi_{n}} \xi_{n}^{2 m-1} \varphi\left(\frac{\xi}{R}\right)}{\left(1+\left|\xi^{\prime}\right|^{2}\right) p(x, i \xi)} d \xi d y^{\prime} \\
= & \int_{B_{r}^{\prime}\left(\hat{x}^{\prime}\right)} \int \frac{e^{i\left(x^{\prime}-y^{\prime}\right) \cdot \xi^{\prime}} e^{i x_{n} \xi_{n}} \xi_{n}^{2 m-1} \varphi\left(\frac{\xi}{R}\right)}{\left(1+\left|\xi^{\prime}\right|^{2}\right) p(x, i \xi)} d \xi d y^{\prime} \\
& -\int_{\partial B_{r}^{\prime}\left(\hat{x}^{\prime}\right)}\left\langle\nabla_{y^{\prime}} \int \frac{e^{i\left(\left(x^{\prime}-y^{\prime}\right) \cdot \xi^{\prime}\right.} e^{i x_{n} \xi_{n}} \xi_{n}^{2 m-1} \varphi\left(\frac{\xi}{R}\right)}{\left(1+\left|\xi^{\prime}\right|^{2}\right) p(x, i \xi)} d \xi, \frac{y^{\prime}-\hat{x}^{\prime}}{r}\right\rangle d S_{y^{\prime}} \\
= & G_{R}+H_{R} .
\end{aligned}
$$

Now use Lemma 4.2 to get

$$
\left|G_{R}\right| \leq \int_{B_{r}^{\prime}\left(\hat{x}^{\prime}\right)} \frac{1}{\left|x^{\prime}-y^{\prime}\right|^{n-3}} d y^{\prime} \leq C
$$

and

$$
\left|H_{R}\right| \leq C \int_{\partial B_{r}^{\prime}\left(\hat{x}^{\prime}\right)} \frac{1}{\left|x^{\prime}-y^{\prime}\right|^{n-2}} d S_{y^{\prime}} \leq \frac{C}{r^{n-2}} \sigma\left(\partial B_{r}^{\prime}\left(\hat{x}^{\prime}\right)\right) \leq C .
$$

So the lemma is proved.

This finishes the estimation of the auxiliary integrals in half space for the operator $N^{+}$.

4.2. Auxiliary integrals in half space for the operator $H$. We now proceed to prove estimates for the operator $H$.

First, let us be more precise about the contours $\gamma^{-+}\left(\xi^{\prime}\right)$.

We take into account that the $m$ roots of $p^{-}\left(y, i \xi^{\prime}, z\right)$ satisfy $\operatorname{Re}\left(z^{-}\left(y, \xi^{\prime}\right)\right) \leq$ $-\lambda\left(1+\left|\xi^{\prime}\right|\right)$ and $\left|z^{-}\left(y, \xi^{\prime}\right)\right| \leq \Lambda\left(1+\left|\xi^{\prime}\right|\right)$, so we take $\gamma^{-}\left(\xi^{\prime}\right)$ a piecewise smooth contour parametrized by an angle $\theta$ which is the arc of the circle centered at 0 of radius $2 \Lambda\left(1+\left|\xi^{\prime}\right|\right)$ joining the points with real part equal to $\frac{-\lambda}{2}\left(1+\left|\xi^{\prime}\right|\right)$ in counterclockwise sense, followed by the vertical segment joining these two points. Denote by $w\left(\theta, \xi^{\prime}\right)$ the points on this contour.

Similarly, since the $m$ roots of $p^{+}\left(y, i \xi^{\prime}, z\right)$ satisfy $\operatorname{Re}\left(z^{+}\left(y, \xi^{\prime}\right)\right) \geq \lambda\left(1+\left|\xi^{\prime}\right|\right)$ and $\left|z^{+}(y, \xi)\right| \leq \Lambda\left(1+\left|\xi^{\prime}\right|\right)$, we take $\gamma^{+}\left(\xi^{\prime}\right)$ a piecewise smooth contour parametrized by an angle $\phi$ which is the arc of the circle centered at 0 of radius $2 \Lambda\left(1+\left|\xi^{\prime}\right|\right)$ joining 
the points with real part equal to $\frac{\lambda}{2}\left(1+\left|\xi^{\prime}\right|\right)$ in counterclockwise sense, followed by the vertical segment joining these two points. Denote by $z\left(\phi, \xi^{\prime}\right)$ the points on this contour.

The following estimates follow directly from the definition.

We have for all $\xi^{\prime}, y, \theta$ and $\phi$, that

$$
\begin{aligned}
& \frac{\lambda}{2}\left(1+\left|\xi^{\prime}\right|\right) \leq\left|w\left(\theta, \xi^{\prime}\right)-z^{-}\left(y, \xi^{\prime}\right)\right| \leq 2 \Lambda\left(1+\left|\xi^{\prime}\right|\right), \\
& \frac{\lambda}{2}\left(1+\left|\xi^{\prime}\right|\right) \leq\left|z\left(\phi, \xi^{\prime}\right)-z^{+}\left(y, \xi^{\prime}\right)\right| \leq 2 \Lambda\left(1+\left|\xi^{\prime}\right|\right), \\
& \frac{\lambda}{2}\left(1+\left|\xi^{\prime}\right|\right) \leq\left|w\left(\theta, \xi^{\prime}\right)-z\left(\phi, \xi^{\prime}\right)\right| \leq 4 \Lambda\left(1+\left|\xi^{\prime}\right|\right) .
\end{aligned}
$$

For all $\xi^{\prime}, y, \theta$ and $\phi$, and any multi-index $\alpha$, we have

$$
\begin{aligned}
\mid D_{\xi^{\prime}}^{\alpha} w\left(\theta, \xi^{\prime}\right) & \leq C\left(1+\left|\xi^{\prime}\right|\right)^{1-|\alpha|}, & \mid D_{\xi^{\prime}}^{\alpha} z\left(\phi, \xi^{\prime}\right) & \leq C\left(1+\left|\xi^{\prime}\right|\right)^{1-|\alpha|}, \\
\mid D_{\xi^{\prime}}^{\alpha} D_{\theta} w\left(\theta, \xi^{\prime}\right) & \leq C\left(1+\left|\xi^{\prime}\right|\right)^{1-|\alpha|}, & \mid D_{\xi^{\prime}}^{\alpha} D_{\phi} z\left(\phi, \xi^{\prime}\right) & \leq C\left(1+\left|\xi^{\prime}\right|\right)^{1-|\alpha|} .
\end{aligned}
$$

Using these estimates together with the estimates of 2.6 we will prove the following lemma.

Lemma 4.5. For $\beta=\beta_{1}+\beta_{2}$, consider the function

$$
\Phi_{\beta}\left(\xi^{\prime}, x, y\right)=\int_{\gamma^{+}\left(\xi^{\prime}\right)} \int_{\gamma^{-}\left(\xi^{\prime}\right)} \frac{e^{-y_{n} z} e^{x_{n} w} w^{\left|\beta_{1}\right|}\left(i \xi^{\prime}\right)^{\beta_{2}}}{p^{+}\left(y, i \xi^{\prime}, z\right) p^{-}\left(y, i \xi^{\prime}, w\right)(w-z)} d w d z .
$$

We have

$$
\left|D_{\xi^{\prime}}^{\gamma} \Phi_{\beta}\left(\xi^{\prime}, x, y\right)\right| \leq C \frac{e^{-\frac{\left(x_{n}+y_{n}\right)\left(1+\left|\xi^{\prime}\right|\right)}{2}}}{\left(1+\left|\xi^{\prime}\right|\right)^{2 m-1+|\gamma|-|\beta|}},
$$

with $C$ depending only on $K_{0}, \lambda$ and $|\beta|$.

Proof. To prove this claim, note that we can write $\Phi_{\beta}\left(\xi^{\prime}, x, y\right)$ as a sum of four terms of the form

$$
\int_{\theta} \int_{\phi} \frac{e^{-y_{n} z} e^{x_{n} w} w^{\left|\beta_{1}\right|}\left(i \xi^{\prime}\right)^{\beta_{2}}}{p^{+}\left(y, i \xi^{\prime}, z\right) p^{-}\left(y, i \xi^{\prime}, w\right)(w-z)} z_{\phi} w_{\theta} d \phi d \theta
$$

for appropriate limits of integration in $\phi$ and $\theta$ that do not depend on $\xi^{\prime}$ and where $z=z\left(\phi, \xi^{\prime}\right)$ and $w=w\left(\theta, \xi^{\prime}\right)$.

Hence, it is enough to estimate

$$
\left|D_{\xi^{\prime}}^{\gamma}\left(\frac{e^{-y_{n} z} e^{x_{n} w} w^{\left|\beta_{1}\right|}\left(i \xi^{\prime}\right)^{\beta_{2}}}{p^{+}\left(y, i \xi^{\prime}, z\right) p^{-}\left(y, i \xi^{\prime}, w\right)(w-z)} z_{\phi} w_{\theta}\right)\right| .
$$

Write the integrand as $\frac{g_{1} g_{2}}{g_{3}}$, where $g_{1}=e^{-y_{n} z} e^{x_{n} w} w^{\left|\beta_{1}\right|}\left(i \xi^{\prime}\right)^{\beta_{2}}, g_{2}=z_{\phi} w_{\theta}$ and $g_{3}=p^{+}\left(y, i \xi^{\prime}, z\right) p^{-}\left(y, i \xi^{\prime}, w\right)(w-z)$. By direct computation we obtain that

$$
\left|D_{\xi^{\prime}}^{\gamma} g_{1}\right| \leq C \frac{e^{-\frac{\left(x_{n}+y_{n}\right)\left(1+\left|\xi^{\prime}\right|\right)}{2}}}{\left(1+\left|\xi^{\prime}\right|\right)^{|\gamma|-|\beta|}}, \quad\left|D_{\xi^{\prime}}^{\gamma} g_{2}\right| \leq C\left(1+\left|\xi^{\prime}\right|\right)^{2-|\gamma|} .
$$

Also note that by estimate 2.6 we have

$$
\left|D_{\xi^{\prime}}^{\gamma}\left(s_{k}\left(x, \xi^{\prime}\right)\left(z\left(\phi, \xi^{\prime}\right)\right)^{m-k}\right)\right| \leq C\left(1+\left|\xi^{\prime}\right|\right)^{m-|\gamma|},
$$


and hence

$$
\left|D_{\xi^{\prime}}^{\gamma} p^{+}\left(x, i \xi^{\prime}, z\left(\phi, \xi^{\prime}\right)\right)\right| \leq C\left(1+\left|\xi^{\prime}\right|\right)^{m-|\gamma|}
$$

This implies that

$$
\left|D_{\xi^{\prime}}^{\gamma} g_{3}\right| \leq C\left(1+\left|\xi^{\prime}\right|\right)^{2 m+1-|\gamma|}
$$

and hence

$$
\left|D_{\xi^{\prime}}^{\gamma}\left(g_{3}\right)^{-1}\right| \leq C \frac{1}{\left(1+\left|\xi^{\prime}\right|\right)^{2 m+1+|\gamma|}} .
$$

The claim is a direct consequence of the estimates above.

With the aid of Lemma 4.5 and the estimates 2.6$)$ and (2.7) the following lemma holds.

Lemma 4.6. Let

$$
\Psi\left(\xi^{\prime}, x, y\right)=\int_{\gamma^{+}\left(\xi^{\prime}\right)} \int_{\gamma^{-}\left(\xi^{\prime}\right)} \frac{e^{-y_{n} z} e^{x_{n} w} p\left(x, i \xi^{\prime}, w\right)}{a(y) p^{+}\left(y, i \xi^{\prime}, z\right) p^{-}\left(y, i \xi^{\prime}, w\right)(w-z)} d w d z .
$$

We have

$$
\left|D_{\xi^{\prime}}^{\alpha} \Psi\left(\xi^{\prime}, x, y\right)\right| \leq C K_{\epsilon ; \delta}|x-y|^{\delta} \frac{e^{-\frac{\left(x_{n}+y_{n}\right)\left(1+\left|\xi^{\prime}\right|\right)}{2}}}{\left(1+\left|\xi^{\prime}\right|\right)^{-1+|\alpha|}} .
$$

Let

$$
\begin{aligned}
& \Theta\left(\xi^{\prime}, x, y, \bar{x}\right) \\
& \quad=\int_{\gamma^{+}\left(\xi^{\prime}\right)} \int_{\gamma^{-}\left(\xi^{\prime}\right)} \frac{e^{-y_{n} z} e^{x_{n} w} p\left(x, i \xi^{\prime}, w\right)\left(p^{-}\left(\bar{x}, i \xi^{\prime}, w\right)-p^{-}\left(y, \xi^{\prime}, w\right)\right)}{a(y)(w-z) p^{+}\left(y, i \xi^{\prime}, z\right) p^{-}\left(y, i \xi^{\prime}, w\right) p^{-}\left(\bar{x}, i \xi^{\prime}, w\right)} d w d z .
\end{aligned}
$$

We have

$$
\left|D_{\xi^{\prime}}^{\alpha} \Theta\left(\xi^{\prime}, x, y, \bar{x}\right)\right| \leq C K_{\epsilon ; \delta} \frac{|\bar{x}-y|^{\delta} e^{-\frac{\left(x_{n}+y_{n}\right)\left(1+\left|\xi^{\prime}\right|\right)}{2}}}{\left(1+\left|\xi^{\prime}\right|\right)^{-1+|\alpha|}} .
$$

The constant $C$ in both cases depends on $K_{0}, \gamma$ and $n$.

Proof. To prove the first estimate note that

$$
\int_{\gamma^{-}} \frac{e^{x_{n} w} p\left(y, \xi^{\prime}, w\right)}{p^{-}\left(y, i \xi^{\prime}, w\right)(w-z)} d w=\int_{\gamma^{-}} \frac{e^{x_{n} w} p^{+}\left(y, \xi^{\prime}, w\right)}{w-z} d w=0,
$$

since for fixed $z \in \gamma^{+}, f(w):=\frac{e^{x_{n} w} p^{+}\left(y, \xi^{\prime}, w\right)}{w-z}$ is analytic inside $\gamma^{-}$.

Hence,

$$
\Psi\left(\xi^{\prime}, x, y\right)=\int_{\gamma^{+}\left(\xi^{\prime}\right)} \int_{\gamma^{-}\left(\xi^{\prime}\right)} \frac{e^{-y_{n} z} e^{x_{n} w}\left(p\left(x, i \xi^{\prime}, w\right)-p\left(y, i \xi^{\prime}, w\right)\right)}{a(y) p^{+}\left(y, i \xi^{\prime}, z\right) p^{-}\left(y, i \xi^{\prime}, w\right)(w-z)} d w d z .
$$

The proof of the lemma is hence a direct application of Lemma 4.5

In the second estimate we have to use (2.7).

To continue, we prove 
Lemma 4.7. Suppose $s>0, n-2 m+|\beta|>0$, and

$$
\left|D_{\xi^{\prime}}^{\alpha} \phi_{\beta}\left(\xi^{\prime}, s\right)\right| \leq C \frac{e^{-s\left(1+\left|\xi^{\prime}\right|\right)}}{\left(1+\left|\xi^{\prime}\right|\right)^{2 m-1+|\alpha|-|\beta|}} .
$$

Then

$$
\left|\int e^{i y^{\prime} \cdot \xi^{\prime}} \phi_{\beta}\left(\xi^{\prime}, s\right) d \xi^{\prime}\right| \leq C \frac{1}{\left(\left|y^{\prime}\right|+s\right)^{n-2 m+|\beta|}} .
$$

Proof. To prove this, write

$$
\begin{aligned}
\int e^{i y^{\prime} \cdot \xi^{\prime}} \Phi_{\beta}\left(\xi^{\prime}, s\right) d \xi^{\prime}= & \frac{1}{\left|y^{\prime}\right|^{2 k}} \int e^{i y^{\prime} \cdot \xi^{\prime}}\left(-\Delta_{\xi^{\prime}}\right)^{k} \Phi_{\beta}\left(\xi^{\prime}, s\right) d \xi^{\prime} \\
= & \frac{1}{\left|y^{\prime}\right|^{2 k}} \int\left(-\Delta_{\xi^{\prime}}\right)^{k}\left(e^{i y^{\prime} \cdot \xi^{\prime}} \varphi\left(\left|y^{\prime}\right| \xi^{\prime}\right)\right) \Phi_{\beta}\left(\xi^{\prime}, s\right) d \xi^{\prime} \\
& +\frac{1}{\left|y^{\prime}\right|^{2 k}} \int e^{i y^{\prime} \cdot \xi^{\prime}}\left(-\Delta_{\xi^{\prime}}\right)^{k}\left(\Phi_{\beta}\left(\xi^{\prime}, s\right)\right)\left(1-\varphi\left(\left|y^{\prime}\right| \xi^{\prime}\right)\right) d \xi^{\prime} \\
= & A+B .
\end{aligned}
$$

We can estimate

$$
\left.|A| \leq C \int_{\left|\xi^{\prime}\right| \leq \frac{2}{\left|y^{\prime}\right|}} \Phi_{\beta}\left(\xi^{\prime}, s\right)\right) d \xi^{\prime} \leq C \int_{\left|\xi^{\prime}\right| \leq \frac{2}{\left|y^{\prime}\right|}} \frac{e^{-\frac{s\left(1+\left|\xi^{\prime}\right|\right)}{2}}}{\left(1+\left|\xi^{\prime}\right|\right)^{2 m-1-|\beta|}} d \xi^{\prime}=: Q
$$

Now

$$
Q \leq C \int_{\left|\xi^{\prime}\right| \leq \frac{2}{\left|y^{\prime}\right|}} \frac{1}{\left(1+\left|\xi^{\prime}\right|\right)^{2 m-1-|\beta|}} d \xi^{\prime} \leq \frac{C}{\left|y^{\prime}\right|^{n-2 m+|\beta|}} .
$$

Also, setting $\zeta^{\prime}=s \xi^{\prime}$, we get

$$
Q \leq \frac{s^{2 m-1-|\beta|}}{s^{n-1}} \int_{\left|\zeta^{\prime}\right| \leq \frac{2 s}{\left|y^{\prime}\right|}} \frac{e^{-\frac{\left|\zeta^{\prime}\right|}{2}}}{\left(s+\left|\zeta^{\prime}\right|\right)^{2 m-1-|\beta|}} d \zeta^{\prime} .
$$

Since $2 m-1-|\beta|<n-1$, we get

$$
Q \leq C \frac{1}{s^{n-2 m+|\beta|}} \int_{R^{n-1}} \frac{e^{-\frac{\left|\zeta^{\prime}\right|}{2}}}{\left|\zeta^{\prime}\right|^{2 m-1-|\beta|}} d \zeta^{\prime} \leq C \frac{1}{s^{n-2 m+|\beta|}} .
$$

So

$$
|A| \leq C \min \left\{\frac{1}{\left|y^{\prime}\right|^{n-2 m+|\beta|}}, \frac{1}{s^{n-2 m+|\beta|}}\right\} \leq C \frac{1}{\left(\left|y^{\prime}\right|+s\right)^{n-2 m+|\beta|}} .
$$

Let us now estimate $B$ :

$$
|B| \leq \frac{1}{\left|y^{\prime}\right|^{2 k}} \int_{\left|\xi^{\prime}\right| \geq \frac{1}{\left|y^{\prime}\right|}} \frac{e^{\frac{-s\left(1+\left|\xi^{\prime}\right|\right)}{2}}}{\left(1+\left|\xi^{\prime}\right|\right)^{2 m-1-|\beta|+2 k}} d \xi^{\prime}=: Q .
$$

Now

$$
Q \leq \frac{1}{\left|y^{\prime}\right|^{2 k}} \int_{\left|\xi^{\prime}\right| \geq \frac{1}{\left|y^{\prime}\right|}} \frac{1}{\left(1+\left|\xi^{\prime}\right|\right)^{2 m-1-|\beta|+2 k}} d \xi^{\prime} \leq C \frac{1}{\left|y^{\prime}\right|^{n-2 m+|\beta|}}
$$


We may assume $\frac{1}{s} \leq \frac{1}{\left|y^{\prime}\right|}$ since otherwise we are done. Setting $\zeta^{\prime}=s \xi^{\prime}$, we get

$$
Q \leq C \frac{1}{s^{n-2 m+|\beta|}}\left(\frac{s}{\left|y^{\prime}\right|}\right)^{2 k} \int_{\left|\zeta^{\prime}\right| \geq \frac{s}{\left|y^{\prime}\right|}} \frac{e^{-\frac{\left|\zeta^{\prime}\right|}{2}}}{\left(s+\left|\zeta^{\prime}\right|\right)^{2 m-1-|\beta|+2 k}} d \zeta^{\prime} .
$$

Noting that $\rho:=\frac{s}{\left|y^{\prime}\right|} \geq 1$ we get

$$
Q \leq C \frac{1}{s^{n-2 m+|\beta|}} \rho^{2 k} \int_{\left|\zeta^{\prime}\right| \geq \rho} e^{-\frac{\left|\zeta^{\prime}\right|}{2}} d \zeta^{\prime} \leq C \frac{1}{s^{n-2 m+|\beta|}}
$$

hence we have

$$
|B| \leq C \frac{1}{\left(\left|y^{\prime}\right|+s\right)^{n-2 m+|\beta|}},
$$

and the lemma is proved.

Now we combine the results in Lemmas 4.5 and 4.6 with Lemma 4.7 to prove

Lemma 4.8. For any multi-index $\beta$ such that $n-2 m+|\beta|>0$ we have

$$
\begin{gathered}
\left|\int e^{i\left(x^{\prime}-y^{\prime}\right) \cdot \xi^{\prime}} \Phi_{\beta}\left(\xi^{\prime}, x, y\right) d \xi^{\prime}\right| \leq C \frac{1}{\left(\left|x^{\prime}-y^{\prime}\right|+\left(x_{n}+y_{n}\right)\right)^{n-2 m+|\beta|}}, \\
\left|\int e^{i\left(x^{\prime}-y^{\prime}\right) \cdot \xi^{\prime}} \Psi\left(\xi^{\prime}, x, y\right) d \xi^{\prime}\right| \leq C K_{\epsilon ; \delta} \frac{|x-y|^{\delta}}{\left(\left|x^{\prime}-y^{\prime}\right|+x_{n}+y_{n}\right)^{n}},
\end{gathered}
$$

and

$$
\left|\int e^{i\left(\bar{x}^{\prime}-y^{\prime}\right) \cdot \xi^{\prime}} \Theta\left(\xi^{\prime}, x, y, \bar{x}\right) d \xi^{\prime}\right| \leq C K_{\epsilon ; \delta} \frac{|\bar{x}-y|^{\delta}}{\left(\left|\bar{x}^{\prime}-y^{\prime}\right|+x_{n}+y_{n}\right)^{n}} .
$$

The constant $C$ depends only on $K_{0}, \lambda$ and $n$.

Proof. The proof follows immediately.

We use the result of Lemma 4.8 to prove the following estimates.

Lemma 4.9. For $x, \bar{x} \in B_{\epsilon}^{+}$and $x_{n} \geq \bar{x}_{n}$, consider

$$
\begin{aligned}
I=\int_{R_{+}^{n}} \eta_{\epsilon}(y) \int e^{i\left(\bar{x}^{\prime}-y^{\prime}\right) \cdot \xi^{\prime}} \int_{\gamma^{+}\left(\xi^{\prime}\right)} & \int_{\gamma^{-}\left(\xi^{\prime}\right)} \\
& \frac{e^{-y_{n} z} e^{x_{n} w} p\left(x, i \xi^{\prime}, w\right)}{a(y) p^{+}\left(y, i \xi^{\prime}, z\right) p^{-}\left(y, i \xi^{\prime}, w\right)(w-z)} d w d z d \xi^{\prime} d y .
\end{aligned}
$$

Then we have

$$
|I| \leq C K_{\epsilon ; \delta}
$$


Proof. Write

$$
\begin{aligned}
I= & \int_{R_{+}^{n}} \eta_{\epsilon}(y) \int e^{i\left(\bar{x}^{\prime}-y^{\prime}\right) \cdot \xi^{\prime}} \int_{\gamma^{+}\left(\xi^{\prime}\right)} \int_{\gamma^{-}\left(\xi^{\prime}\right)} \frac{e^{-y_{n} z} e^{x_{n} w} p\left(x, i \xi^{\prime}, w\right)}{a(\bar{x}) p^{+}\left(\bar{x}, i \xi^{\prime}, z\right) p^{-}\left(\bar{x}, i \xi^{\prime}, w\right)(w-z)} d w d z d \xi^{\prime} d y \\
& \left.+\int_{R_{+}^{n}} \eta_{\epsilon}(y) \int e^{i\left(\bar{x}^{\prime}-y^{\prime}\right) \cdot \xi^{\prime}} \int_{\gamma^{+}\left(\xi^{\prime}\right)} \int_{\gamma^{-}\left(\xi^{\prime}\right)} \frac{e^{-y_{n} z} e^{x_{n} w} p\left(x, i \xi^{\prime}, w\right)}{(w-z)}\right) d w d z d \xi^{\prime} d y . \\
& \left(\frac{1}{a(y) p^{+}\left(y, i \xi^{\prime}, z\right) p^{-}\left(y, i \xi^{\prime}, w\right)}-\frac{1}{a(\bar{x}) p^{+}\left(\bar{x}, i \xi^{\prime}, z\right) p^{-}\left(\bar{x}, i \xi^{\prime}, w\right)}\right) \\
= & A+B .
\end{aligned}
$$

Notice that

$$
\begin{aligned}
A= & \int_{R_{+}^{n}} \eta_{\epsilon}(y) \int e^{i\left(\bar{x}^{\prime}-y^{\prime}\right) \cdot \xi^{\prime}} \int_{\gamma^{+}\left(\xi^{\prime}\right)} \int_{\gamma^{-}\left(\xi^{\prime}\right)} \frac{e^{-y_{n} z} e^{x_{n} w}\left(p\left(x, i \xi^{\prime}, w\right)-p\left(\bar{x}, i \xi^{\prime}, w\right)\right.}{a(\bar{x}) p^{+}\left(\bar{x}, i \xi^{\prime}, z\right) p^{-}\left(\bar{x}, i \xi^{\prime}, w\right)(w-z)} d w d z d \xi^{\prime} d y \\
= & \int_{R_{+}^{n}} \eta_{\epsilon}(y)\left(1-\Delta_{y^{\prime}}\right) \\
& \int \frac{e^{i\left(\bar{x}^{\prime}-y^{\prime}\right) \cdot \xi^{\prime}}}{1+\left|\xi^{\prime}\right|^{2}} \int_{\gamma^{+}\left(\xi^{\prime}\right)} \int_{\gamma^{-}\left(\xi^{\prime}\right)} \frac{e^{-y_{n} z} e^{x_{n} w}\left(p\left(x, i \xi^{\prime}, w\right)-p\left(\bar{x}, i \xi^{\prime}, w\right)\right.}{a(\bar{x}) p^{+}\left(\bar{x}, i \xi^{\prime}, z\right) p^{-}\left(\bar{x}, i \xi^{\prime}, w\right)(w-z)} d w d z d \xi^{\prime} d y \\
= & \int_{R_{+}^{n}}\left(1-\Delta_{y^{\prime}}\right) \eta_{\epsilon}(y) \\
& \int \frac{e^{i\left(\bar{x}^{\prime}-y^{\prime}\right) \cdot \xi^{\prime}}}{1+\left|\xi^{\prime}\right|^{2}} \int_{\gamma^{+}\left(\xi^{\prime}\right)} \int_{\gamma^{-}\left(\xi^{\prime}\right)} \frac{e^{-y_{n} z} e^{x_{n} w}\left(p\left(x, i \xi^{\prime}, w\right)-p\left(\bar{x}, i \xi^{\prime}, w\right)\right.}{a(\bar{x}) p^{+}\left(\bar{x}, i \xi^{\prime}, z\right) p^{-}\left(\bar{x}, i \xi^{\prime}, w\right)(w-z)} d w d z d \xi^{\prime} d y .
\end{aligned}
$$

Hence, by Lemma 4.8 ,

$$
\begin{aligned}
|A| & \leq C K_{\epsilon ; \delta}|x-\bar{x}|^{\delta} \frac{1}{\epsilon^{2}} \int_{B_{\epsilon}^{+}} \frac{1}{\left(\left|\bar{x}^{\prime}-y^{\prime}\right|+x_{n}+y_{n}\right)^{n-2}} d y \\
& \leq C K_{\epsilon ; \delta}|x-\bar{x}|^{\delta} \frac{1}{\epsilon^{2}} \int_{B_{\epsilon}^{+}} \frac{1}{\left(\left|\bar{x}^{\prime}-y^{\prime}\right|+\bar{x}_{n}+y_{n}\right)^{n-2}} d y \leq C K_{\epsilon ; \delta}|x-\bar{x}|^{\delta} \leq C K_{\epsilon ; \delta} \epsilon^{\delta} .
\end{aligned}
$$

Next write

$$
B=C_{1}+C_{2}+C_{3},
$$

where

$$
\begin{aligned}
C_{1}=\int_{R_{+}^{n}} \eta_{\epsilon}(y) \int e^{i\left(\bar{x}^{\prime}-y^{\prime}\right) \cdot \xi^{\prime}} \int_{\gamma^{+}\left(\xi^{\prime}\right)} & \int_{\gamma^{-}\left(\xi^{\prime}\right)} \frac{e^{-y_{n} z} e^{x_{n} w} p\left(x, i \xi^{\prime}, w\right)}{p^{-}\left(\bar{x}, i \xi^{\prime}, w\right)(w-z)} \\
& \times \frac{\left(p^{-}\left(\bar{x}, i \xi^{\prime}, w\right)-p^{-}\left(y, i \xi^{\prime}, w\right)\right)}{a(y) p^{+}\left(y, i \xi^{\prime}, z\right) p^{-}\left(y, i \xi^{\prime}, w\right)} d w d z d \xi^{\prime} d y
\end{aligned}
$$

and $C_{2}$ and $C_{3}$ are similar terms. 
Again use Lemma 4.8 to get

$$
\begin{aligned}
\left|C_{1}\right| & \leq C K_{\epsilon ; \delta} \int_{R_{+}^{n}} \eta_{\epsilon}(y) \frac{|\bar{x}-y|^{\delta}}{\left(\left|\bar{x}^{\prime}-y^{\prime}\right|+x_{n}+y_{n}\right)^{n}} d y \\
& \leq C K_{\epsilon ; \delta} \int_{R_{+}^{n}} \eta_{\epsilon}(y) \frac{|\bar{x}-y|^{\delta}}{\left(\left|\bar{x}^{\prime}-y^{\prime}\right|+\bar{x}_{n}+y_{n}\right)^{n}} d y \leq C K_{\epsilon ; \delta} \epsilon^{\delta} .
\end{aligned}
$$

This finishes the estimation of $I$.

Finally, we consider an auxiliary integral over a ball in half space.

Lemma 4.10. Let

$$
\begin{aligned}
I_{B}=\int_{B_{\rho}^{+}(\hat{x})} \eta_{\epsilon}(y) \int e^{i\left(\bar{x}^{\prime}-y^{\prime}\right) \cdot \xi^{\prime}} \int_{\gamma^{+}\left(\xi^{\prime}\right)} \int_{\gamma^{-}\left(\xi^{\prime}\right)} \\
\\
\frac{e^{-y_{n} z} e^{x_{n} w}\left(p\left(x, i \xi^{\prime}, w\right)-p\left(\bar{x}, i \xi^{\prime}, w\right)\right)}{(w-z) a(\bar{x}) p^{+}\left(\bar{x}, i \xi^{\prime}, z\right) p^{-}\left(\bar{x}, i \xi^{\prime}, w\right)} d w d z d \xi^{\prime} d y .
\end{aligned}
$$

Then we have that

$$
\left|I_{B}\right| \leq C K_{\epsilon ; \delta}|x-\bar{x}|^{\delta}
$$

Proof.

$$
\begin{aligned}
& I_{B}=\int_{B_{\rho}^{+}(\hat{x})}\left(\eta_{\epsilon}(y)-\eta_{\epsilon}(\bar{x})\right) \int e^{i\left(\bar{x}^{\prime}-y^{\prime}\right) \cdot \xi^{\prime}} \int_{\gamma^{+}\left(\xi^{\prime}\right)} \int_{\gamma^{-}\left(\xi^{\prime}\right)} \\
& \frac{e^{-y_{n} z} e^{x_{n} w}\left(p\left(x, i \xi^{\prime}, w\right)-p\left(\bar{x}, i \xi^{\prime}, w\right)\right)}{(w-z) a(\bar{x}) p^{+}\left(\bar{x}, i \xi^{\prime}, z\right) p^{-}\left(\bar{x}, i \xi^{\prime}, w\right)} d w d z d \xi^{\prime} d y \\
& +\eta_{\epsilon}(\bar{x}) \int_{B_{\rho}^{+}(\hat{x})} \int e^{i\left(\bar{x}^{\prime}-y^{\prime}\right) \cdot \xi^{\prime}} \int_{\gamma^{+}\left(\xi^{\prime}\right)} \int_{\gamma^{-}\left(\xi^{\prime}\right)} \\
& =A+\eta_{\epsilon}(\bar{x}) B \text {. } \\
& \frac{e^{-y_{n} z} e^{x_{n} w}\left(p\left(x, i \xi^{\prime}, w\right)-p\left(\bar{x}, i \xi^{\prime}, w\right)\right)}{(w-z) a(\bar{x}) p^{+}\left(\bar{x}, i \xi^{\prime}, z\right) p^{-}\left(\bar{x}, i \xi^{\prime}, w\right)} d w d z d \xi^{\prime} d y
\end{aligned}
$$

By Lemma 4.8 we have

$$
\begin{aligned}
|A| & \leq C K_{\epsilon ; \delta}|x-\bar{x}|^{\delta} \frac{1}{\epsilon} \int_{B_{\rho}^{+}(\hat{x})}|y-\bar{x}| \frac{1}{\left(\left|\bar{x}^{\prime}-y^{\prime}\right|+x_{n}+y_{n}\right)^{n}} d y \\
& \leq C K_{\epsilon ; \delta}|x-\bar{x}|^{\delta} \frac{1}{\epsilon} \int_{B_{\rho}^{+}(\hat{x})}|y-\bar{x}| \frac{1}{\left(\left|\bar{x}^{\prime}-y^{\prime}\right|+\bar{x}_{n}+y_{n}\right)^{n}} d y \\
& \leq C K_{\epsilon ; \delta}|x-\bar{x}|^{\delta} \frac{\rho}{\epsilon} \leq C K_{\epsilon ; \delta}|x-\bar{x}|^{\delta} .
\end{aligned}
$$


To estimate the term $B$, note that

$$
\begin{aligned}
B= & \int_{B_{\rho}^{+}(\hat{x})}\left(1-\Delta_{y^{\prime}}\right) \int \frac{e^{i\left(\bar{x}^{\prime}-y^{\prime}\right) \cdot \xi^{\prime}}}{1+\left|\xi^{\prime}\right|^{2}} \int_{\gamma^{+}\left(\xi^{\prime}\right)} \int_{\gamma^{-}\left(\xi^{\prime}\right)} \\
= & \frac{e^{-y_{n} z} e^{x_{n} w}\left(p\left(x, i \xi^{\prime}, w\right)-p\left(\bar{x}, i \xi^{\prime}, w\right)\right)}{(w-z) a(\bar{x}) p^{+}\left(\bar{x}, i \xi^{\prime}, z\right) p^{-}\left(\bar{x}, i \xi^{\prime}, w\right)} d w d z d \xi^{\prime} d y \\
& \int_{B_{\rho}^{+}(\hat{x})} \int \frac{e^{i\left(\bar{x}^{\prime}-y^{\prime}\right) \cdot \xi^{\prime}}}{1+\left|\xi^{\prime}\right|^{2}} \int_{\gamma^{+}\left(\xi^{\prime}\right)} \int_{\gamma^{-}\left(\xi^{\prime}\right)} \frac{e^{-y_{n} z} e^{x_{n} w}\left(p\left(x, i \xi^{\prime}, w\right)-p\left(\bar{x}, i \xi^{\prime}, w\right)\right)}{(w-z) a(\bar{x}) p^{+}\left(\bar{x}, i \xi^{\prime}, z\right) p^{-}\left(\bar{x}, i \xi^{\prime}, w\right)} d w d z d \xi^{\prime} d y \\
& -\int_{\partial B_{\rho}^{+}(\hat{x}) \cap R_{+}^{n}} Q d S_{y} \\
= & C_{1}-C_{2},
\end{aligned}
$$

where

$$
\begin{aligned}
& Q=\nabla_{y^{\prime}}\left(\int \frac{e^{i\left(\bar{x}^{\prime}-y^{\prime}\right) \cdot \xi^{\prime}}}{1+\left|\xi^{\prime}\right|^{2}} \int_{\gamma^{+}\left(\xi^{\prime}\right)} \int_{\gamma^{-}\left(\xi^{\prime}\right)} \frac{e^{-y_{n} z} e^{x_{n} w}\left(p\left(x, i \xi^{\prime}, w\right)-p\left(\bar{x}, i \xi^{\prime}, w\right)\right)}{(w-z) a(\bar{x}) p^{+}\left(\bar{x}, i \xi^{\prime}, z\right) p^{-}\left(\bar{x}, i \xi^{\prime}, w\right)} d w d z d \xi^{\prime}\right) \\
& \times \frac{y^{\prime}-\hat{x}^{\prime}}{\rho} .
\end{aligned}
$$

We have, again by Lemma 4.8 .

$$
\begin{aligned}
\left|C_{1}\right| & \leq C K_{\epsilon ; \delta}|x-\bar{x}|^{\delta} \int_{B_{\rho}^{+}(\hat{x})} \frac{1}{\left(\left|\bar{x}^{\prime}-y^{\prime}\right|+x_{n}+y_{n}\right)^{n-2}} d y \\
& \leq C K_{\epsilon ; \delta}|x-\bar{x}|^{\delta} \int_{B_{\rho}^{+}(\hat{x})} \frac{1}{\left(\left|\bar{x}^{\prime}-y^{\prime}\right|+\bar{x}_{n}+y_{n}\right)^{n-2}} d y \leq C K_{\epsilon ; \delta}|x-\bar{x}|^{\delta}
\end{aligned}
$$

and

$$
\begin{aligned}
C_{2} & \leq C K_{\epsilon ; \delta}|x-\bar{x}|^{\delta} \int_{\partial B_{\rho}^{+}(\hat{x}) \cap R_{+}^{n}} \frac{1}{\left(\left|\bar{x}^{\prime}-y^{\prime}\right|+x_{n}+y_{n}\right)^{n-1}} d S_{y} \\
& \leq C K_{\epsilon ; \delta}|x-\bar{x}|^{\delta} \int_{\partial B_{\rho}^{+}(\hat{x}) \cap R_{+}^{n}} \frac{1}{\left(\left|\bar{x}^{\prime}-y^{\prime}\right|+\bar{x}_{n}+y_{n}\right)^{n-1}} d S_{y} \\
& \leq C K_{\epsilon ; \delta}|x-\bar{x}|^{\delta} \int_{\partial B_{\rho}^{+}(\hat{x}) \cap R_{+}^{n}} \frac{1}{|\bar{x}-y|^{n-1}} d S_{y} \leq C K_{\epsilon ; \delta}|x-\bar{x}|^{\delta} .
\end{aligned}
$$

We are now ready for the main results of this section.

\subsection{Main results in half space.}

Lemma 4.11. Define

$$
u:=N^{+}\left(g \eta_{\epsilon}\right)
$$

Then, $u \in C^{2}\left(B_{\epsilon}^{+}\right)$. 
Proof. The proof is a standard modification of the proof of Lemma 3.4. By using a cutoff function one shows that for $|\alpha| \leq 2 m$, we have for $x \in B_{\epsilon}^{+}$:

$$
D^{\alpha}\left(N^{+}\left(g \eta_{\epsilon}\right)\right)(x)=\lim _{R \rightarrow \infty} \int_{R_{+}^{n}} g(y) \eta_{\epsilon}(y) \int \frac{e^{i(x-y) \cdot \xi(i \xi)^{\alpha} \varphi\left(\frac{\xi}{R}\right)}}{p(y, i \xi)} d \xi d y .
$$

By Lemma 4.11, we have

$$
L\left(N^{+}\left(g \eta_{\epsilon}\right)\right)(x)=\lim _{R \rightarrow \infty} \int_{R_{+}^{n}} g(y) \eta_{\epsilon}(y) \int \frac{e^{i(x-y) \cdot \xi} p(x, i \xi) \varphi\left(\frac{\xi}{R}\right)}{p(y, i \xi)} d \xi d y .
$$

Therefore,

$$
\begin{aligned}
L\left(N^{+}\left(g \eta_{\epsilon}\right)\right)(x) & -g(x) \eta_{\epsilon}(x) \\
= & \lim _{R \rightarrow \infty} \int_{R_{+}^{n}} g(y) \eta_{\epsilon}(y) \int \frac{e^{i(x-y) \cdot \xi}(p(x, i \xi)-p(y, i \xi)) \varphi\left(\frac{\xi}{R}\right)}{p(y, i \xi)} d \xi d y .
\end{aligned}
$$

Define

$$
T\left(g \eta_{\epsilon}\right)(x)=L\left(N^{+}\left(g \eta_{\epsilon}\right)\right)(x)-g(x) \eta_{\epsilon}(x) .
$$

By repeating the same proof for free space, this time using the auxiliary integrals in half space, we can prove the following theorem.

Theorem 4.12. There exists a constant $C$ depending only on $K_{0}$ and $\lambda$ such that

$$
\left|T\left(g \eta_{\epsilon}\right)\right|_{\delta ; B_{\epsilon}^{+}} \leq C K_{\delta ; \epsilon}|g|_{\delta ; B_{\epsilon}^{+}} .
$$

Proof. The proof is exactly the same as the proof of Theorem 3.7 using this time the auxiliary integrals in Lemmas 4.3 and 4.4

Now we can show that $u:=N^{+}\left(g \eta_{\epsilon}\right) \in C^{2 m+\delta}\left(B_{\epsilon}^{+}\right)$.

\section{Lemma 4.13.}

$$
u:=N^{+}\left(g \eta_{\epsilon}\right) \in C^{2 m+\delta}\left(B_{\epsilon}^{+}\right) .
$$

Proof. By exactly the same proof as in Lemma 3.5 one shows that for any $|\alpha| \leq 2 m$ except $\alpha=2 m e_{n}$, we have

$$
D^{\alpha} N^{+}\left(g \eta_{\epsilon}\right) \in C^{\delta}\left(B_{\epsilon}^{+}\right) .
$$

To show that $\frac{\partial^{2 m} N^{+}\left(g \eta_{\epsilon}\right)}{\partial x_{n}^{2 m}} \in C^{\delta}\left(B_{\epsilon}^{+}\right)$, we note that

$$
a(x) \frac{\partial^{2 m} N^{+}\left(g \eta_{\epsilon}\right)}{\partial x_{n}^{2 m}}=-\sum_{\alpha \neq 2 m e_{n}} a_{\alpha}(x) D^{\alpha} N^{+}\left(g \eta_{\epsilon}\right)(x)+L\left(N^{+}\left(g \eta_{\epsilon}\right)\right)(x),
$$

which is a sum of two functions in $C^{\delta}\left(B_{\epsilon}^{+}\right)$by Theorem 4.12

Since $a \geq \lambda$ and $a \in C^{\delta}\left(B_{\epsilon}^{+}\right)$, the result follows. 
We now proceed to prove estimates for the operator $H$.

Recall that

$$
\begin{array}{r}
L\left(H\left(g \eta_{\epsilon}\right)\right)(x)=\int_{R_{+}^{n}} g(y) \eta_{\epsilon}(y) \int e^{i\left(x^{\prime}-y^{\prime}\right) \cdot \xi^{\prime}} \int_{\gamma^{+}\left(\xi^{\prime}\right)} \int_{\gamma^{-}\left(\xi^{\prime}\right)} \\
=\int_{R_{+}^{n}} g(y) \eta_{\epsilon}(y) \int e^{\frac{e^{-y_{n} z} e^{x_{n} w} p\left(x, i \xi^{\prime}, w\right)}{a(y) p^{+}\left(y, i \xi^{\prime}, z\right) p^{-}\left(y, i \xi^{\prime}, w\right)(w-z)} \int_{\gamma^{+}\left(\xi^{\prime}\right)} \int_{\gamma^{-}\left(\xi^{\prime}\right)}} d w d z d \xi^{\prime} d y \\
\frac{e^{-y_{n} z} e^{x_{n} w}\left(p\left(x, i \xi^{\prime}, w\right)-p\left(y, i \xi^{\prime}, w\right)\right)}{a(y) p^{+}\left(y, i \xi^{\prime}, z\right) p^{-}\left(y, i \xi^{\prime}, w\right)(w-z)} d w d z d \xi^{\prime} d y,
\end{array}
$$

where the last equality follows since $f(w):=\frac{e^{x_{n} w} p^{+}\left(y, i \xi^{\prime}, w\right)}{w-z}$ is analytic inside $\gamma^{-}\left(\xi^{\prime}\right)$ for each fixed $z \in \gamma^{+}\left(\xi^{\prime}\right)$.

We now prove the main estimate for the operator $H$. Set $F\left(g \eta_{\epsilon}\right)=L\left(H\left(g \eta_{\epsilon}\right)\right)$.

Theorem 4.14. There exists a constant $C$ depending only on $K_{0}$ and $\lambda$ such that

$$
\left|F\left(g \eta_{\epsilon}\right)\right|_{\delta ; B_{\epsilon}^{+}} \leq C K_{\delta ; \epsilon}|g|_{\delta ; B_{\epsilon}^{+}}
$$

Proof. Set

$$
\Psi\left(x, y, \xi^{\prime}\right)=\int_{\gamma^{+}\left(\xi^{\prime}\right)} \int_{\gamma^{-}\left(\xi^{\prime}\right)} \frac{e^{-y_{n} z} e^{x_{n} w}\left(p\left(x, i \xi^{\prime}, w\right)-p\left(y, i \xi^{\prime}, w\right)\right)}{a(y) p^{+}\left(y, i \xi^{\prime}, z\right) p^{-}\left(y, i \xi^{\prime}, w\right)(w-z)} d w d z
$$

Let $x, \bar{x} \in B_{\epsilon}^{+}$, and we may assume $x_{n} \geq \bar{x}_{n}$.

Write

$$
\begin{aligned}
F\left(g \eta_{\epsilon}\right)(x)-F\left(g \eta_{\epsilon}\right)(\bar{x})= & \int_{R_{+}^{n}} g(y) \eta_{\epsilon}(y) \int\left(e^{i\left(x^{\prime}-y^{\prime}\right) \cdot \xi^{\prime}}-e^{i\left(\bar{x}^{\prime}-y^{\prime}\right) \cdot \xi^{\prime}}\right) \Psi\left(x, y, \xi^{\prime}\right) d \xi^{\prime} d y \\
& +\int_{R_{+}^{n}} g(y) \eta_{\epsilon}(y) \int e^{i\left(\bar{x}^{\prime}-y^{\prime}\right) \cdot \xi^{\prime}}\left(\Psi\left(x, y, \xi^{\prime}\right)-\Psi\left(\bar{x}, y, \xi^{\prime}\right)\right) d \xi^{\prime} d y \\
= & A+B
\end{aligned}
$$

Let's estimate the term $A$. Write

$$
\begin{aligned}
A= & \int_{R_{+}^{n}}(g(y)-g(\bar{x})) \eta_{\epsilon}(y) \int\left(e^{i\left(x^{\prime}-y^{\prime}\right) \cdot \xi^{\prime}}-e^{i\left(\bar{x}^{\prime}-y^{\prime}\right) \cdot \xi^{\prime}}\right) \Psi\left(x, y, \xi^{\prime}\right) d \xi^{\prime} d y \\
& +g(\bar{x}) \int_{R_{+}^{n}} \eta_{\epsilon}(y) \int\left(e^{i\left(x^{\prime}-y^{\prime}\right) \cdot \xi^{\prime}}-e^{i\left(\bar{x}^{\prime}-y^{\prime}\right) \cdot \xi^{\prime}}\right) \Psi\left(x, y, \xi^{\prime}\right) d \xi^{\prime} d y \\
= & A_{1}+g(\bar{x}) A_{2}
\end{aligned}
$$


Now,

$$
\begin{aligned}
A_{1}= & \int_{B_{\rho}^{+}(\hat{x})}(g(y)-g(\bar{x})) \eta_{\epsilon}(y) \int e^{i\left(x^{\prime}-y^{\prime}\right) \cdot \xi^{\prime}} \Psi\left(x, y, \xi^{\prime}\right) d \xi^{\prime} d y \\
& -\int_{B_{\rho}^{+}(\hat{x})}(g(y)-g(\bar{x})) \eta_{\epsilon}(y) \int e^{i\left(\bar{x}^{\prime}-y^{\prime}\right) \cdot \xi^{\prime}} \Psi\left(x, y, \xi^{\prime}\right) d \xi^{\prime} d y \\
& +\int_{R_{+}^{n} \backslash B_{\rho}^{+}(\hat{x})}(g(y)-g(\bar{x})) \eta_{\epsilon}(y) \int\left(e^{i\left(x^{\prime}-y^{\prime}\right) \cdot \xi^{\prime}}-e^{i\left(\bar{x}^{\prime}-y^{\prime}\right) \cdot \xi^{\prime}}\right) \Psi\left(x, y, \xi^{\prime}\right) d \xi^{\prime} d y \\
= & B_{1}-B_{2}+B_{3},
\end{aligned}
$$

and we estimate, using Lemma 4.8

$$
\begin{aligned}
& \left|B_{1}\right| \leq C K_{\delta ; \epsilon}[g] \int_{B_{\rho}^{+}(\hat{x})}|y-\bar{x}|^{\delta} \frac{|y-x|^{\delta}}{\left(\left|x^{\prime}-y^{\prime}\right|+x_{n}+y_{n}\right)^{n}} d y \leq C K_{\delta ; \epsilon}[g]|x-\bar{x}|^{\delta}, \\
& \left|B_{2}\right| \leq C K_{\delta ; \epsilon}[g] \int_{B_{\rho}^{+}(\hat{x})}|y-\bar{x}|^{\delta} \frac{|y-x|^{\delta}}{\left(\left|\bar{x}^{\prime}-y^{\prime}\right|+x_{n}+y_{n}\right)^{n}} d y \leq C K_{\delta ; \epsilon}[g]|x-\bar{x}|^{\delta},
\end{aligned}
$$

where we have used that $x_{n} \geq \bar{x}_{n}$, and

$$
B_{3}=\sum_{k=1}^{n-1}\left(x_{k}-\bar{x}_{k}\right) \int_{0}^{1} \int_{R_{+}^{n} \backslash B_{\rho}^{+}(\hat{x})}(g(y)-g(\bar{x})) \eta_{\epsilon}(y) \int e^{i\left(x_{s}^{\prime}-y^{\prime}\right) \cdot \xi^{\prime}} \xi_{k} \Psi\left(x, y, \xi^{\prime}\right) d \xi^{\prime} d y d s .
$$

Hence, using Lemma 4.8, we have

$$
\begin{aligned}
\left|B_{3}\right| & \leq C K_{\delta ; \epsilon}[g]|x-\bar{x}| \int_{0}^{1} \int_{R_{+}^{n} \backslash B_{\rho}^{+}(\hat{x})}|y-\bar{x}|^{\delta}|y-x|^{\delta} \frac{1}{\left(\left|x_{s}^{\prime}-y^{\prime}\right|+x_{n}+y_{n}\right)^{n+1}} d y d s \\
& \leq C K_{\delta ; \epsilon}[g]|x-\bar{x}| \int_{R_{+}^{n} \backslash B_{\rho}^{+}(\hat{x})} \frac{|\bar{x}-y|^{\delta}}{|\bar{x}-y|^{n+1}} d y \\
& \leq C K_{\delta ; \epsilon}[g]|x-\bar{x}|^{\delta} .
\end{aligned}
$$

Next,

$$
\begin{aligned}
A_{2}= & \int_{B_{\rho}^{+}(\hat{x})} \eta_{\epsilon}(y) \int e^{i\left(x^{\prime}-y^{\prime}\right) \cdot \xi^{\prime}} \Psi\left(x, y, \xi^{\prime}\right) d \xi^{\prime} d y \\
& -\int_{B_{\rho}^{+}(\hat{x})} \eta_{\epsilon}(y) \int e^{i\left(\bar{x}^{\prime}-y^{\prime}\right) \cdot \xi^{\prime}} \Psi\left(x, y, \xi^{\prime}\right) d \xi^{\prime} d y \\
& +\int_{R_{+}^{n} \backslash B_{\rho}^{+}(\hat{x})} \eta_{\epsilon}(y) \int\left(e^{i\left(x^{\prime}-y^{\prime}\right) \cdot \xi^{\prime}}-e^{i\left(\bar{x}^{\prime}-y^{\prime}\right) \cdot \xi^{\prime}}\right) \Psi\left(x, y, \xi^{\prime}\right) d \xi^{\prime} d y \\
= & C_{1}-C_{2}+C_{3} .
\end{aligned}
$$

Now estimate $C_{1}$ :

$$
\left|C_{1}\right| \leq C K_{\delta ; \epsilon} \int_{B_{\rho}^{+}(\hat{x})} \frac{|x-y|^{\delta}}{|y-x|^{n}} d y \leq C K_{\delta ; \epsilon}|x-\bar{x}|^{\delta} .
$$


For $C_{2}$, we have

$$
\begin{aligned}
C_{2}= & \int_{B_{\rho}^{+}(\hat{x})} \eta_{\epsilon}(y) \int e^{i\left(\bar{x}^{\prime}-y^{\prime}\right) \cdot \xi^{\prime}} \int_{\gamma^{+}\left(\xi^{\prime}\right)} \int_{\gamma^{-}\left(\xi^{\prime}\right)} \frac{e^{-y_{n} z} e^{x_{n} w} p\left(x, i \xi^{\prime}, w\right)}{a(\bar{x})(w-z) p^{+}\left(\bar{x}, i \xi^{\prime}, z\right) p^{-}\left(\bar{x}, i \xi^{\prime}, w\right)} d w d z d \xi^{\prime} d y \\
& +\int_{B_{\rho}^{+}(\hat{x})} \eta_{\epsilon}(y) \int e^{i\left(\bar{x}^{\prime}-y^{\prime}\right) \cdot \xi^{\prime}} \int_{\gamma^{+}\left(\xi^{\prime}\right)} \int_{\gamma^{-}\left(\xi^{\prime}\right)} \frac{e^{-y_{n} z} e^{x_{n} w} p\left(x, i \xi^{\prime}, w\right)}{w-z} \\
& \times\left(\frac{1}{a(y) p^{+}\left(y, i \xi^{\prime}, z\right) p^{-}\left(y, i \xi^{\prime}, w\right)}-\frac{1}{a(\bar{x}) p^{+}\left(\bar{x}, i \xi^{\prime}, z\right) p^{-}\left(\bar{x}, i \xi^{\prime}, w\right)}\right) d w d z d \xi^{\prime} d y \\
= & D_{1}+D_{2} .
\end{aligned}
$$

We can write

$$
\begin{array}{r}
D_{1}=\int_{B_{\rho}^{+}(\hat{x})} \eta_{\epsilon}(y) \int e^{i\left(\bar{x}^{\prime}-y^{\prime}\right) \cdot \xi^{\prime}} \int_{\gamma^{+}\left(\xi^{\prime}\right)} \int_{\gamma^{-}\left(\xi^{\prime}\right)} \frac{e^{-y_{n} z} e^{x_{n} w}\left(p\left(x, i \xi^{\prime}, w\right)-p\left(\bar{x}, i \xi^{\prime}, w\right)\right)}{a(\bar{x})(w-z) p^{+}\left(\bar{x}, i \xi^{\prime}, z\right) p^{-}\left(\bar{x}, i \xi^{\prime}, w\right)} \\
d w d z d \xi^{\prime} d y
\end{array}
$$

and estimate

$$
\left|D_{1}\right| \leq C K_{\delta ; \epsilon}|x-\bar{x}|^{\delta},
$$

by the estimations of Lemma 4.10 Also,

$$
\left|D_{2}\right| \leq C K_{\delta ; \epsilon} \int_{B_{\rho}^{+}(\hat{x})} \frac{|\bar{x}-y|^{\delta}}{\left(\left|\bar{x}^{\prime}-y^{\prime}\right|+x_{n}+y_{n}\right)^{n}} d y \leq C K_{\delta ; \epsilon}|x-\bar{x}|^{\delta},
$$

again by Lemma 4.10 and using that $x_{n} \geq \bar{x}_{n}$.

The term $C_{3}$ is estimated exactly as the term $B_{3}$. This finishes the estimation of the term $A$.

For the estimation of the term $B$, we write

$$
\begin{aligned}
B= & \int_{R_{+}^{n}}(g(y)-g(\bar{x})) \eta_{\epsilon}(y) \int e^{i\left(\bar{x}^{\prime}-y^{\prime}\right) \cdot \xi^{\prime}}\left(\Psi\left(x, y, \xi^{\prime}\right)-\Psi\left(\bar{x}, y, \xi^{\prime}\right)\right) d \xi^{\prime} d y \\
& +g(\bar{x}) \int_{R_{+}^{n}} \eta_{\epsilon}(y) \int e^{i\left(\bar{x}^{\prime}-y^{\prime}\right) \cdot \xi^{\prime}}\left(\Psi\left(x, y, \xi^{\prime}\right)-\Psi\left(\bar{x}, y, \xi^{\prime}\right)\right) d \xi^{\prime} d y \\
= & E_{1}+g(\bar{x}) E_{2}
\end{aligned}
$$

and

$$
E_{1}=F_{1}+F_{2}
$$

where

$$
\begin{aligned}
F_{1}=\int_{R_{+}^{n}}(g(y)-g(\bar{x})) \eta_{\epsilon}(y) & \int e^{i\left(\bar{x}^{\prime}-y^{\prime}\right) \cdot \xi^{\prime}} \int_{\gamma^{+}\left(\xi^{\prime}\right)} \int_{\gamma^{-}\left(\xi^{\prime}\right)} \\
& \frac{e^{-y_{n} z} e^{x_{n} w}\left(p\left(x, i \xi^{\prime}, w\right)-p\left(\bar{x}, i \xi^{\prime}, w\right)\right)}{a(y)(w-z) p^{+}\left(y, i \xi^{\prime}, z\right) p^{-}\left(y, i \xi^{\prime}, w\right)} d w d z d \xi^{\prime} d y
\end{aligned}
$$


and

$$
\begin{aligned}
F_{2}=\int_{R_{+}^{n}}(g(y)-g(\bar{x})) \eta_{\epsilon}(y) \int e^{i\left(\bar{x}^{\prime}-y^{\prime}\right) \cdot \xi^{\prime}} \int_{\gamma^{+}\left(\xi^{\prime}\right)} \int_{\gamma^{-}\left(\xi^{\prime}\right)} \\
\\
\frac{e^{-y_{n} z}\left(e^{x_{n} w}-e^{\bar{x}_{n} w}\right) p\left(\bar{x}, i \xi^{\prime}, w\right)}{a(y)(w-z) p^{+}\left(y, i \xi^{\prime}, z\right) p^{-}\left(y, i \xi^{\prime}, w\right)} d w d z d \xi^{\prime} d y .
\end{aligned}
$$

We have

$$
\left|F_{1}\right| \leq C K_{\delta ; \epsilon}[g]|\bar{x}-x|^{\delta} \int \eta_{\epsilon}(y) \frac{|\bar{x}-y|^{\delta}}{\left(\left|\bar{x}^{\prime}-y^{\prime}\right|+x_{n}+y_{n}\right)^{n}} d y \leq C K_{\delta ; \epsilon}[g]|\bar{x}-x|^{\delta}
$$

since $x_{n} \geq \bar{x}_{n}$. Next, write

$$
F_{2}=G_{1}-G_{2}+G_{3}
$$

where

$$
\begin{aligned}
& G_{1}=\int_{B_{\rho}^{+}(\hat{x})}(g(y)-g(\bar{x})) \eta_{\epsilon}(y) \int e^{i\left(\bar{x}^{\prime}-y^{\prime}\right) \cdot \xi^{\prime}} \int_{\gamma^{+}\left(\xi^{\prime}\right)} \int_{\gamma^{-}} \\
& \frac{e^{-y_{n} z} e^{x_{n} w}\left(p\left(\bar{x}, i \xi^{\prime}, w\right)-p\left(y, i \xi^{\prime}, w\right)\right)}{a(y)(w-z) p^{+}\left(y, i \xi^{\prime}, z\right) p^{-}\left(y, i \xi^{\prime}, w\right)} d w d z d \xi^{\prime} d y \\
& G_{2}=\int_{B_{\rho}^{+}(\hat{x})}(g(y)-g(\bar{x})) \eta_{\epsilon}(y) \int e^{i\left(\bar{x}^{\prime}-y^{\prime}\right) \cdot \xi^{\prime}} \int_{\gamma^{+}\left(\xi^{\prime}\right)} \int_{\gamma^{-}\left(\xi^{\prime}\right)} \\
& \frac{e^{-y_{n} z} e^{\bar{x}_{n} w}\left(p\left(\bar{x}, i \xi^{\prime}, w\right)-p\left(y, i \xi^{\prime}, w\right)\right)}{a(y)(w-z) p^{+}\left(y, i \xi^{\prime}, z\right) p^{-}\left(y, i \xi^{\prime}, w\right)} d w d z d \xi^{\prime} d y
\end{aligned}
$$

and

$$
\begin{array}{r}
G_{3}=\int_{R_{+}^{n} \backslash B_{\rho}^{+}(\hat{x})}(g(y)-g(\bar{x})) \eta_{\epsilon}(y) \int e^{i\left(\bar{x}^{\prime}-y^{\prime}\right) \cdot \xi^{\prime}} \int_{\gamma^{+}\left(\xi^{\prime}\right)} \int_{\gamma^{-}\left(\xi^{\prime}\right)} \\
\frac{e^{-y_{n} z}\left(e^{x_{n} w}-e^{\bar{x}_{n} w}\right) p\left(\bar{x}, i \xi^{\prime}, w\right)}{a(y)(w-z) p^{+}\left(y, i \xi^{\prime}, z\right) p^{-}\left(y, i \xi^{\prime}, w\right)} d w d z d \xi^{\prime} d y
\end{array}
$$

and we estimate, using Lemma 4.8 .

$$
\left|G_{1}\right| \leq C K_{\delta ; \epsilon}[g] \int_{B_{\rho}^{+}(\hat{x})} \frac{|\bar{x}-y|^{\delta}}{\left(\left|\bar{x}^{\prime}-y^{\prime}\right|+x_{n}+y_{n}\right)^{n}} d y \leq C K_{\delta ; \epsilon}[g]|\bar{x}-x|^{\delta}
$$

since $x_{n} \geq \bar{x}_{n}$. In exactly the same way we get

$$
\left|G_{2}\right| \leq C K_{\delta ; \epsilon}[g]|\bar{x}-x|^{\delta}
$$

and

$$
\left|G_{3}\right| \leq C K_{\delta ; \epsilon}[g]|\bar{x}-x| \int_{R_{+}^{n} \backslash B_{\rho}^{+}(\hat{x})} \frac{|\bar{x}-y|^{\delta}}{\left(\left|\bar{x}^{\prime}-y^{\prime}\right|+\bar{x}_{n}+y_{n}\right)^{n+1}} d y \leq C K_{\delta ; \epsilon}[g]|\bar{x}-x|^{\delta} .
$$

As for $E_{2}$, we proceed in a similar fashion:

$$
E_{2}=H_{1}+H_{2}
$$


where

$$
\begin{aligned}
H_{1}=\int_{R_{+}^{n}} \eta_{\epsilon}(y) \int e^{i\left(\bar{x}^{\prime}-y^{\prime}\right) \cdot \xi^{\prime}} & \int_{\gamma^{+}\left(\xi^{\prime}\right)} \int_{\gamma^{-}\left(\xi^{\prime}\right)} \\
& \frac{e^{-y_{n} z} e^{x_{n} w}\left(p\left(x, i \xi^{\prime}, w\right)-p\left(\bar{x}, i \xi^{\prime}, w\right)\right.}{a(y)(w-z) p^{+}\left(y, i \xi^{\prime}, z\right) p^{-}\left(y, i \xi^{\prime}, w\right)} d w d z d \xi^{\prime} d y
\end{aligned}
$$

and

$$
\begin{aligned}
H_{2}=\int_{R_{+}^{n}} \eta_{\epsilon}(y) \int e^{i\left(\bar{x}^{\prime}-y^{\prime}\right) \cdot \xi^{\prime}} & \int_{\gamma^{+}\left(\xi^{\prime}\right)} \int_{\gamma^{-}\left(\xi^{\prime}\right)} \\
& \frac{e^{-y_{n} z}\left(e^{x_{n} w}-e^{\bar{x}_{n} w}\right) p\left(\bar{x}, i \xi^{\prime}, w\right)}{a(y)(w-z) p^{+}\left(y, i \xi^{\prime}, z\right) p^{-}\left(y, i \xi^{\prime}, w\right)} d w d z d \xi^{\prime} d y .
\end{aligned}
$$

We have

$$
H_{1}=J_{1}+J_{2}
$$

where

$$
\begin{aligned}
J_{1}=\int_{R_{+}^{n}} \eta_{\epsilon}(y) \int e^{i\left(\bar{x}^{\prime}-y^{\prime}\right) \cdot \xi^{\prime}} & \int_{\gamma^{+}\left(\xi^{\prime}\right)} \int_{\gamma^{-}\left(\xi^{\prime}\right)} \\
& \frac{e^{-y_{n} z} e^{x_{n} w}\left(p\left(x, i \xi^{\prime}, w\right)-p\left(\bar{x}, i \xi^{\prime}, w\right)\right.}{a(\bar{x})(w-z) p^{+}\left(\bar{x}, i \xi^{\prime}, z\right) p^{-}\left(\bar{x}, i \xi^{\prime}, w\right)} d w d z d \xi^{\prime} d y
\end{aligned}
$$

and

$$
J_{2}=\int_{R_{+}^{n}} \eta_{\epsilon}(y) \int e^{i\left(\bar{x}^{\prime}-y^{\prime}\right) \cdot \xi^{\prime}} X d \xi^{\prime} d y
$$

with

$$
\begin{aligned}
& X=\int_{\gamma^{+}\left(\xi^{\prime}\right)} \int_{\gamma^{-}\left(\xi^{\prime}\right)} \frac{e^{-y_{n} z} e^{x_{n} w}\left(p\left(x, i \xi^{\prime}, w\right)-p\left(\bar{x}, i \xi^{\prime}, w\right)\right)}{w-z} \\
& \times\left(\frac{1}{a(y) p^{+}\left(y, i \xi^{\prime}, z\right) p^{-}\left(y, i \xi^{\prime}, w\right)}-\frac{1}{a(\bar{x}) p^{+}\left(\bar{x}, i \xi^{\prime}, z\right) p^{-}\left(\bar{x}, i \xi^{\prime}, w\right)}\right) d w d z d \xi^{\prime} d y
\end{aligned}
$$

We have

$$
\left|J_{1}\right| \leq C K_{\delta ; \epsilon}|\bar{x}-x|^{\delta} .
$$

Using the argument at the beginning of the proof of Lemma 4.9 and using Lemma 4.8 we have

$$
\left|J_{2}\right| \leq C \epsilon^{\delta} K_{\delta ; \epsilon}^{2}|\bar{x}-x|^{\delta} \leq C K_{\delta ; \epsilon}|x-\bar{x}|^{\delta} .
$$

Finally, write

$$
H_{2}=K_{1}+K_{2}+K_{3},
$$


where

$$
\begin{aligned}
& K_{1}=\int_{B_{\rho}^{+}(\hat{x})} \eta_{\epsilon}(y) \int e^{i\left(\bar{x}^{\prime}-y^{\prime}\right) \cdot \xi^{\prime}} \int_{\gamma^{+}\left(\xi^{\prime}\right)} \int_{\gamma^{-}\left(\xi^{\prime}\right)} \\
& \frac{e^{-y_{n} z} e^{x_{n} w}\left(p\left(\bar{x}, i \xi^{\prime}, w\right)-p\left(y, i \xi^{\prime}, w\right)\right)}{a(y)(w-z) p^{+}\left(y, i \xi^{\prime}, z\right) p^{-}\left(y, i \xi^{\prime}, w\right)} d w d z d \xi^{\prime} d y . \\
& K_{2}=\int_{B_{\rho}^{+}(\hat{x})} \eta_{\epsilon}(y) \int e^{i\left(\bar{x}^{\prime}-y^{\prime}\right) \cdot \xi^{\prime}} \int_{\gamma^{+}\left(\xi^{\prime}\right)} \int_{\gamma^{-}\left(\xi^{\prime}\right)} \\
& \frac{e^{-y_{n} z} e^{\bar{x}_{n} w}\left(p\left(\bar{x}, i \xi^{\prime}, w\right)-p\left(y, i \xi^{\prime}, w\right)\right)}{a(y)(w-z) p^{+}\left(y, i \xi^{\prime}, z\right) p^{-}\left(y, i \xi^{\prime}, w\right)} d w d z d \xi^{\prime} d y . \\
& K_{3}=\int_{R_{+}^{n} \backslash B_{\rho}^{+}(\hat{x})} \eta_{\epsilon}(y) \int e^{i\left(\bar{x}^{\prime}-y^{\prime}\right) \cdot \xi^{\prime}} \int_{\gamma^{+}\left(\xi^{\prime}\right)} \int_{\gamma^{-}\left(\xi^{\prime}\right)} \\
& \frac{e^{-y_{n} z}\left(e^{\bar{x}_{n} w}-e^{x_{n} w}\right)\left(p\left(\bar{x}, i \xi^{\prime}, w\right)-p\left(y, i \xi^{\prime}, w\right)\right)}{a(y)(w-z) p^{+}\left(y, i \xi^{\prime}, z\right) p^{-}\left(y, i \xi^{\prime}, w\right)} d w d z d \xi^{\prime} d y .
\end{aligned}
$$

Note that

$$
\left|K_{1}\right|,\left|K_{2}\right| \leq C K_{\delta ; \epsilon} \int_{B_{\rho}^{+}(\hat{x})} \frac{|\bar{x}-y|^{\delta}}{\left(\left|\bar{x}^{\prime}-y^{\prime}\right|+\bar{x}_{n}+y_{n}\right)^{n}} \leq C K_{\delta ; \epsilon}|\bar{x}-x|^{\delta}
$$

and

$$
\left|K_{3}\right| \leq C K_{\delta ; \epsilon}|\bar{x}-x| \int_{R_{+}^{n} \backslash B_{\rho}^{+}(\hat{x})} \frac{|\bar{x}-y|^{\delta}}{\left(\left|\bar{x}^{\prime}-y^{\prime}\right|+\bar{x}_{n}+y_{n}\right)^{n+1}} \leq C K_{\delta ; \epsilon}|\bar{x}-x|^{\delta} .
$$

This finishes the proof that

$$
\left[F\left(g \eta_{\epsilon}\right)\right]_{\delta ; B_{\epsilon}^{+}} \leq C K_{\delta ; \epsilon}|g|_{\delta ; B_{\epsilon}^{+}} .
$$

The proof that $\left|F\left(g \eta_{\epsilon}\right)\right|_{0 ; B_{\epsilon}^{+}} \leq C K_{\delta ; \epsilon}|g|_{0 ; B_{\epsilon}^{+}}$is straightforward and we omit it.

We also have

Lemma 4.15. The function

$$
u:=H\left(g \eta_{\epsilon}\right) \in C^{2 m+\delta}\left(B_{\epsilon}^{+}\right) .
$$

Proof. One shows using Lemmas 4.9 and 4.10 that for $\alpha \neq 2 m e_{n}$, we have $D^{\alpha} u \in$ $C^{2 m+\delta}\left(B_{\epsilon}^{+}\right)$.

Then observe that

$$
a(x) \frac{\partial^{2 m} H\left(g \eta_{\epsilon}\right)}{\partial x_{n}^{2 m}}=-\sum_{\alpha \neq 2 m e_{n}} a_{\alpha}(x) D^{\alpha} H\left(g \eta_{\epsilon}\right)(x)+L\left(H\left(g \eta_{\epsilon}\right)\right)(x),
$$

which is a sum of two functions in $C^{\delta}\left(B_{\epsilon}^{+}\right)$by Theorem 4.14

Since $a \geq \lambda$ and $a \in C^{\delta}\left(B_{\epsilon}^{+}\right)$, the result follows.

In order to analyze the boundary values we need two lemmas.

Lemma 4.16. For $k=0, \ldots, m-1$, we have

$$
\int_{\gamma^{-}\left(\xi^{\prime}\right)} \frac{w^{k}}{p^{-}\left(y, i \xi^{\prime}, w\right)(w-z)} d w=\frac{-2 \pi i z^{k}}{p^{-}\left(y, i \xi^{\prime}, z\right)} .
$$


Proof. Let $C_{R}$ denote the circle of radius $R$ centered at the origin parametrized counterclockwise. Let $C_{R}^{-}$denote the closed contour which is the part of $C_{R}$ on the left half plane followed by the vertical segment joining $-i R$ with $i R$ and let $C_{R}^{+}$denote the closed contour which is the vertical segment joining $i R$ with $-i R$ followed by the part of $C_{R}$ on the right half plane.

For $k=0, \ldots, m-1$ we have that for large enough $R$,

$$
\int_{C_{R}} \frac{w^{k}}{p^{-}\left(y, i \xi^{\prime}, w\right)(w-z)} d w=0 .
$$

Hence,

$$
\begin{aligned}
\int_{\gamma^{-}\left(\xi^{\prime}\right)} \frac{w^{k}}{p^{-}\left(y, i \xi^{\prime}, w\right)(w-z)} d w & =\int_{C_{R}^{-}} \frac{w^{k}}{p^{-}\left(y, i \xi^{\prime}, w\right)(w-z)} d w \\
& =-\int_{C_{R}^{+}} \frac{w^{k}}{p^{-}\left(y, i \xi^{\prime}, w\right)(w-z)} d w=\frac{-2 \pi i z^{k}}{p^{-}\left(y, i \xi^{\prime}, z\right)},
\end{aligned}
$$

where the last equality follows from Cauchy's formula.

Lemma 4.17. Let $r>0$ and assume $y_{n}>r$ and $0<x_{n}<\frac{r}{2}$. Then for any $k$ and $2 m+2 l-k \geq n+2$ we have

$$
\begin{aligned}
\frac{1}{|x-y|^{2 l}} \int e^{i(x-y) \cdot \xi}\left(-\Delta_{\xi}\right)^{l} & \left(\frac{\left(i \xi_{n}\right)^{k}}{p(y, i \xi)}\right) d \xi \\
& =\int e^{i\left(x^{\prime}-y^{\prime}\right) \cdot \xi^{\prime}} \int_{\gamma^{+}\left(\xi^{\prime}\right)} e^{\left(x_{n}-y_{n}\right) z} \frac{-z^{k}}{p\left(y, i \xi^{\prime}, z\right)} d z d \xi^{\prime} .
\end{aligned}
$$

Proof. Write

$$
\begin{aligned}
& \int e^{i\left(x^{\prime}-y^{\prime}\right) \cdot \xi^{\prime}} \int_{\gamma^{+}\left(\xi^{\prime}\right)} e^{\left(x_{n}-y_{n}\right) z} \frac{-z^{k}}{p\left(y, i \xi^{\prime}, z\right)} d z d \xi^{\prime} \\
& \quad-\frac{1}{|x-y|^{2 l}} \int e^{i(x-y) \cdot \xi}\left(-\Delta_{\xi}\right)^{l}\left(\frac{\left(i \xi_{n}\right)^{k}}{p(y, i \xi)}\right) d \xi \\
&=-\int e^{i\left(x^{\prime}-y^{\prime}\right) \cdot \xi^{\prime}} \int_{\gamma^{+}\left(\xi^{\prime}\right)} e^{\left(x_{n}-y_{n}\right) z} \frac{-z^{k}}{p\left(y, i \xi^{\prime}, z\right)} d z d \xi^{\prime} \\
&-\int e^{i\left(x^{\prime}-y^{\prime}\right) \cdot \xi^{\prime}} \varphi\left(\frac{\xi^{\prime}}{R}\right) \int_{\gamma^{+}\left(\xi^{\prime}\right)} e^{\left(x_{n}-y_{n}\right) z} \frac{-z^{k}}{p\left(y, i \xi^{\prime}, z\right)} d z d \xi^{\prime} \\
&+\int e^{i\left(x^{\prime}-y^{\prime}\right) \cdot \xi^{\prime}} \varphi\left(\frac{\xi^{\prime}}{R}\right) \int_{\gamma^{+}\left(\xi^{\prime}\right)} e^{\left(x_{n}-y_{n}\right) z} \frac{-z^{k}}{p\left(y, i \xi^{\prime}, z\right)} d z d \xi^{\prime} \\
&-\int e^{i\left(x^{\prime}-y^{\prime}\right) \cdot \xi^{\prime}} \varphi\left(\frac{\xi^{\prime}}{R}\right) \int_{-\infty}^{\infty} \frac{e^{\left(x_{n}-y_{n}\right) i \xi_{n}}\left(i \xi_{n}\right)^{k}}{p\left(y, i \xi^{\prime}, i \xi_{n}\right)} d \xi_{n} d \xi^{\prime} \\
&+\int e^{i\left(x^{\prime}-y^{\prime}\right) \cdot \xi^{\prime}} \varphi\left(\frac{\xi^{\prime}}{R}\right) \int_{-\infty}^{\infty} \frac{e^{\left(x_{n}-y_{n}\right) i \xi_{n}}\left(i \xi_{n}\right)^{k}}{p\left(y, i \xi^{\prime}, i \xi_{n}\right)} d \xi_{n} d \xi^{\prime} \\
&-\int \frac{e^{i(x-y) \cdot \xi}\left(i \xi_{n}\right)^{k} \varphi\left(\frac{\xi^{\prime}}{R}\right) \varphi\left(\frac{\xi_{n}}{R}\right)}{p(y, i \xi)} d \xi^{\prime} d \xi_{n}
\end{aligned}
$$




$$
\begin{aligned}
& +\int \frac{e^{i(x-y) \cdot \xi}\left(i \xi_{n}\right)^{k} \varphi\left(\frac{\xi^{\prime}}{R}\right) \varphi\left(\frac{\xi_{n}}{\bar{R}}\right)}{p(y, i \xi)} d \xi^{\prime} d \xi_{n} \\
& -\frac{1}{|x-y|^{2 l}} \int e^{i(x-y) \cdot \xi}\left(-\Delta_{\xi}\right)^{l}\left(\frac{\left(i \xi_{n}\right)^{k}}{p(y, i \xi)}\right) d \xi \\
& =A+B+C+D .
\end{aligned}
$$

We show that given $\epsilon>0$, we can choose $\bar{R} \geq R \geq 1$ to make each term smaller than $\epsilon$.

We have

$$
|A| \leq C \int_{\left|\xi^{\prime}\right| \geq R} e^{\frac{-r\left(1+\left|\xi^{\prime}\right|\right)}{4}} d \xi^{\prime} .
$$

By a simple change of contour we see

$$
\begin{aligned}
B & =\int e^{i\left(x^{\prime}-y^{\prime}\right) \cdot \xi^{\prime}} \varphi\left(\frac{\xi^{\prime}}{R}\right)\left(\int_{\gamma^{+}\left(\xi^{\prime}\right)} e^{\left(x_{n}-y_{n}\right) z} \frac{-z^{k}}{p\left(y, i \xi^{\prime}, z\right)} d z-\int_{-\infty}^{\infty} \frac{e^{\left(x_{n}-y_{n}\right) i \xi_{n}}\left(i \xi_{n}\right)^{k}}{p\left(y, i \xi^{\prime}, i \xi_{n}\right)} d \xi_{n}\right) d \xi^{\prime} \\
& =0,
\end{aligned}
$$

and we estimate $C$ as follows:

$$
|C| \leq \int_{\left|\xi^{\prime}\right| \leq 2 R} \int_{\left|\xi_{n}\right| \geq \bar{R}} \frac{1}{\left(1+\left|\xi^{\prime}\right|+\left|\xi_{n}\right|\right)^{2 m-k}} d \xi_{n} d \xi^{\prime} \leq C \frac{R^{n}}{\bar{R}} .
$$

To estimate $D$, first set $B(R, \bar{R}):=\left\{\xi:\left|\xi^{\prime}\right| \leq R,\left|\xi_{n}\right| \leq \bar{R}\right\}$.

$$
\begin{aligned}
D= & \frac{1}{|x-y|^{2 l}} \int e^{i(x-y) \cdot \xi}\left(-\Delta_{\xi}\right)^{l}\left(\frac{\left(i \xi_{n}\right)^{k}}{p(y, i \xi)}\right)\left(1-\varphi\left(\frac{\xi^{\prime}}{R}\right) \varphi\left(\frac{\xi_{n}}{\bar{R}}\right)\right) d \xi \\
& +\frac{1}{|x-y|^{2 l}} \sum_{|\gamma|+|\beta|=2 l,|\gamma| \geq 1} \int e^{i(x-y) \cdot \xi} D^{\beta}\left(\frac{\left(i \xi_{n}\right)^{k}}{p(y, i \xi)}\right) D^{\gamma}\left(\varphi\left(\frac{\xi^{\prime}}{R}\right) \varphi\left(\frac{\xi_{n}}{\bar{R}}\right)\right) d \xi \\
= & D_{1}+D_{2} .
\end{aligned}
$$

We have

$$
\left|D_{1}\right| \leq \frac{1}{|x-y|^{2 l}} \int_{R^{n} \backslash B(R, \bar{R})} \frac{1}{(1+|\xi|)^{2 m+2 l-k}} d \xi \leq C \frac{1}{R}
$$

and

$$
\begin{aligned}
\left|D_{2}\right| & \leq \frac{1}{|x-y|^{2 l}} \sum_{|\gamma|+|\beta|=2 l,|\gamma| \geq 1} \frac{C}{R^{\gamma}} \int_{B(2 R, 2 \bar{R}) \backslash B(R, \bar{R})} \frac{1}{(1+|\xi|)^{2 m+|\beta|-k}} d \xi \\
& \leq C \frac{R^{n+1-2 m+k}}{R^{2 l}} \leq \frac{C}{R}
\end{aligned}
$$

where we have used that $\bar{R} \geq R \geq 1$ and $2 l \geq n+2-2 m+k$.

To finish the proof, we choose $R \geq 1$ large enough depending on $\epsilon$ and $r$ to make $|A|,|D|<\epsilon$ and then choose $\bar{R} \geq R$, to make $|C|<\epsilon$.

We will use the previous two lemmas to prove 
Lemma 4.18. Given $g \in C^{\delta}\left(B_{\epsilon}^{+}\right)$, we define for $x \in B_{\epsilon}^{+}$the function

$$
u(x)=N^{+}\left(g \eta_{\epsilon}\right)(x)-H\left(g \eta_{\epsilon}\right)(x) .
$$

Then

$$
\frac{\partial^{k} u\left(x^{\prime}, 0\right)}{\partial x_{n}^{k}}=0
$$

for $k=0, \ldots, m-1$.

Proof. Fix $r>0$. Let $0<x_{n}<\frac{r}{2}$ and write

$$
\begin{aligned}
\frac{\partial^{k} u\left(x^{\prime}, x_{n}\right)}{\partial x_{n}^{k}}= & \left(\lim _{R \rightarrow \infty} \int_{R_{+}^{n}} \eta_{\epsilon}(y) g(y) \int \frac{e^{i(x-y) \cdot \xi} \varphi\left(\frac{\xi}{R}\right)\left(i \xi_{n}\right)^{k}}{p(y, i \xi)} d \xi d y\right) \\
& -\int_{R_{+}^{n}} \eta_{\epsilon}(y) g(y) \int e^{i\left(x^{\prime}-y^{\prime}\right) \cdot \xi^{\prime}} \int_{\gamma^{+}\left(\xi^{\prime}\right)} \frac{e^{-y_{n} z}}{a(y) p^{+}\left(y, i \xi^{\prime}, z\right)} \\
& \int_{\gamma^{-}\left(\xi^{\prime}\right)} \frac{e^{x_{n} w} w^{k}}{(w-z) p\left(y, i \xi^{\prime}, w\right)} d w d z d \xi^{\prime} d y \\
= & \left(\lim _{R \rightarrow \infty} \int_{R_{+}^{n} \cap\left\{y_{n} \leq r\right\}} \cdots\right)-\int_{R_{+}^{n} \cap\left\{y_{n} \leq r\right\}} \ldots \\
& +\left(\lim _{R \rightarrow \infty} \int_{R_{+}^{n} \cap\left\{y_{n}>r\right\}} \cdots\right)-\int_{R_{+}^{n} \cap\left\{y_{n}>r\right\}} \cdots \\
= & E_{0}\left(r, x_{n}\right)+E_{1}\left(r, x_{n}\right) .
\end{aligned}
$$

It follows from Lemmas 3.1 and 4.8 that for some $C$ independent of $x$, we have

$$
\left|E_{0}\left(r, x_{n}\right)\right| \leq C r .
$$

Next, we claim

$$
\left|E_{1}\left(r, x_{n}\right)\right| \leq C x_{n}
$$

We have, using the same argument as in Lemma 3.4 that

$$
\begin{aligned}
& \lim _{R \rightarrow \infty} \int_{R_{+}^{n} \cap\left\{y_{n}>r\right\}} \eta_{\epsilon}(y) g(y) \int \frac{e^{i(x-y) \cdot \xi} \varphi\left(\frac{\xi}{R}\right)\left(i \xi_{n}\right)^{k}}{p(y, i \xi)} d \xi d y \\
& \quad=\int_{R_{+}^{n} \cap\left\{y_{n}>r\right\}} \eta_{\epsilon}(y) g(y) \frac{1}{|x-y|^{2 l}} \int e^{i(x-y) \cdot \xi}\left(-\Delta_{\xi}\right)^{l}\left(\frac{\left(i \xi_{n}\right)^{k}}{p(y, i \xi)}\right) d \xi d y \\
& \quad=\int_{R_{+}^{n} \cap\left\{y_{n}>r\right\}} \eta_{\epsilon}(y) g(y) \int e^{i\left(x^{\prime}-y^{\prime}\right) \cdot \xi^{\prime}} \int_{\gamma^{+}\left(\xi^{\prime}\right)} e^{\left(x_{n}-y_{n}\right) z} \frac{-z^{k}}{p\left(y, i \xi^{\prime}, z\right)} d z d \xi^{\prime} d y
\end{aligned}
$$


where the last equality is by Lemma 4.17. Hence,

$$
\begin{aligned}
E_{1}\left(r, x_{n}\right)= & \int_{R_{+}^{n} \cap\left\{y_{n}>r\right\}} \eta_{\epsilon}(y) g(y) \int e^{i\left(x^{\prime}-y^{\prime}\right) \cdot \xi^{\prime}} \int_{\gamma^{+}\left(\xi^{\prime}\right)} e^{\left(x_{n}-y_{n}\right) z} \frac{-z^{k}}{p\left(y, i \xi^{\prime}, z\right)} d z d \xi^{\prime} d y \\
& -\frac{1}{2 \pi i} \int_{R_{+}^{n} \cap\left\{y_{n}>r\right\}} \eta_{\epsilon}(y) g(y) \int e^{i\left(x^{\prime}-y^{\prime}\right) \cdot \xi^{\prime}} \\
& \quad \int_{\gamma^{+}\left(\xi^{\prime}\right)} \frac{e^{-y_{n} z}}{a(y) p^{+}\left(y, i \xi^{\prime}, z\right)} \int_{\gamma^{-}\left(\xi^{\prime}\right)} \frac{e^{x_{n} w} w^{k}}{(w-z) p\left(y, i \xi^{\prime}, w\right)} d w d z d \xi^{\prime} d y .
\end{aligned}
$$

Rewrite

$$
-E\left(r, x_{n}\right)=A+B+C,
$$

where

$$
\begin{aligned}
A= & \int_{R_{+}^{n} \cap\left\{y_{n}>r\right\}} \eta_{\epsilon}(y) g(y) \int e^{i\left(x^{\prime}-y^{\prime}\right) \cdot \xi^{\prime}} \int_{\gamma^{+}\left(\xi^{\prime}\right)} \frac{\left(e^{\left(x_{n}-y_{n}\right) z}-e^{-y_{n} z}\right) z^{k}}{p\left(y, i \xi^{\prime}, z\right)} d z d \xi^{\prime} d y, \\
B=\frac{1}{2 \pi i} \int_{R_{+}^{n} \cap\left\{y_{n}>r\right\}} \eta_{\epsilon}(y) g(y) \int e^{i\left(x^{\prime}-y^{\prime}\right) \cdot \xi^{\prime}} & \\
& \quad \int_{\gamma^{+}\left(\xi^{\prime}\right)} \frac{e^{-y_{n} z}}{a(y) p^{+}\left(y, i \xi^{\prime}, z\right)} \int_{\gamma^{-}\left(\xi^{\prime}\right)} \frac{\left(e^{x_{n} w}-1\right) w^{k}}{p^{-}\left(y, i \xi^{\prime}, w\right)(w-z)} d w d z d \xi^{\prime} d y
\end{aligned}
$$

and

$$
C=\int_{R_{+}^{n} \cap\left\{y_{n}>r\right\}} \eta_{\epsilon}(y) g(y) \int e^{i\left(x^{\prime}-y^{\prime}\right) \cdot \xi^{\prime}} Q d \xi^{\prime} d y,
$$

where

$$
\begin{aligned}
Q=\int_{\gamma^{+}\left(\xi^{\prime}\right)} \frac{e^{-y_{n} z} z^{k}}{p\left(y, i \xi^{\prime}, z\right)} d z & \\
& \quad+\frac{1}{2 \pi i} \int_{\gamma^{+}\left(\xi^{\prime}\right)} \frac{e^{-y_{n} z}}{a(y) p^{+}\left(y, i \xi^{\prime}, z\right)} \int_{\gamma^{-}\left(\xi^{\prime}\right)} \frac{w^{k}}{p^{-}\left(y, i \xi^{\prime}, w\right)(w-z)} d w d z .
\end{aligned}
$$

Using Lemma 4.8 , it is easy to see that

$$
|A|,|B| \leq C x_{n} e^{\frac{-r\left(1+\left|\xi^{\prime}\right|\right)}{4}}
$$

and using Lemma 4.16 we have $Q=0$ and hence $C=0$. Thus, the claim follows and the proof of the lemma is complete.

We are now ready to prove the main theorem of this section.

Theorem 4.19. Let the operator $L$ satisfy (2.3), (2.1) and (2.2). In addition assume that (2.7) holds. Then there exists $\epsilon>0$ depending on $K_{0}$ and $\lambda$ so that given $f \in C^{\delta}\left(B_{\epsilon}^{+}\right)$, there exists $u \in C^{2 m+\delta}\left(B_{\epsilon}^{+}\right)$such that $L u=f$ in $B_{\frac{\epsilon}{2}}^{+}$and $u$ satisfies the $m$ boundary conditions, $u\left(x^{\prime}, 0\right)=0, \frac{\partial u\left(x^{\prime}, 0\right)}{\partial x_{n}}=0, \ldots, \frac{\partial^{m-1} u\left(x^{\prime}, 0\right)}{\partial x_{n}^{m-1}}=0$.

Let the operator L satisfy (2.3), (2.1) and (2.2). In addition assume that (2.7) holds. Then there exists $\epsilon>0$ depending on $K_{0}$ and $\lambda$ so that given $f \in C^{\delta}\left(\bar{B}_{\epsilon}^{+}\right)$, 
there exists $u \in C^{2 m+\delta}\left(B_{\epsilon}^{+}\right)$such that $L u=f$ in $B_{\frac{\epsilon}{2}}^{+}$and $u$ satisfies the $m$ boundary conditions, $u\left(x^{\prime}, 0\right)=0, \frac{\partial u\left(x^{\prime}, 0\right)}{\partial x_{n}}=0, \ldots, \frac{\partial^{m-1} u\left(x^{\prime}, 0\right)}{\partial x_{n}^{m-1}}=0$.

Proof. Define

$$
T(g)(x)=L(N(g)(x)-H(g)(x))-g(x) .
$$

For $\left.g \in C^{\delta}\left(B_{\epsilon}^{+}\right)\right)$, it follows by Theorem 4.12 and Theorem 4.14 that

$$
\left|T\left(g \eta_{\epsilon}\right)\right|_{\delta ; B_{\epsilon}^{+}} \leq C K_{\delta, \epsilon}|g|_{\delta ; B_{\epsilon}^{+}} .
$$

For $\epsilon$ to be chosen, define the sequence $\left.g_{k} \in C^{\delta}\left(B_{\epsilon}^{+}\right)\right)$by

$$
g_{0}=f \quad \text { and } \quad g_{k+1}=f-T\left(g_{k} \eta_{\epsilon}\right) .
$$

Note that

$$
\begin{aligned}
\left|g_{k+1}-g_{k}\right|_{\delta ; B_{\epsilon}^{+}} & =\left|T\left(\left(g_{k}-g_{k-1}\right) \eta_{\epsilon}\right)\right|_{\delta ; B_{\epsilon}^{+}} \\
& \leq\left. C K_{\delta, \epsilon}\left|g_{k}-g_{k-1}\right|\right|_{\delta ; B_{\epsilon}^{+}} \\
& \leq\left. C \epsilon^{\beta-\delta} K_{\beta, \epsilon}\left|g_{k}-g_{k-1}\right|\right|_{\delta ; B_{\epsilon}^{+}} .
\end{aligned}
$$

Choosing $\epsilon$ small enough so that $C \epsilon^{\beta-\delta} K_{\beta, \epsilon}<1$, by the contraction mapping theorem we can conclude that there exists $\left.g \in C^{\delta}\left(B_{\epsilon}^{+}\right)\right)$such that $g=f-T\left(g \eta_{\epsilon}\right)$. This means that $g\left(1-\eta_{\epsilon}\right)=f-\left(L\left(N\left(g \eta_{\epsilon}\right)-H\left(g \eta_{\epsilon}\right)\right)\right)$ in $B_{\epsilon}^{+}$. And hence,

$$
f=L\left(N\left(g \eta_{\epsilon}\right)-H\left(g \eta_{\epsilon}\right)\right)
$$

in $B_{\frac{\epsilon}{2}}^{+}$.

Let $u=N\left(g \eta_{\epsilon}\right)-H\left(g \eta_{\epsilon}\right)$ in $B_{\frac{\epsilon}{2}}^{+}$. Then $u$ satisfies the conclusion of the theorem.

\section{REFERENCES}

[1] S. Agmon, A. Douglis and L. Nirenberg, Estimates near the boundary for solutions of elliptic partial differential equations satisfying general boundary conditions. I, Comm. Pure Appl. Math. 12 (1959), 623-727. MR 0125307.

[2] A. P. Calderón, Lecture Notes on Pseudo-differential Operators and Elliptic Boundary Value Problems. Cursos de Matemática, No. 1. Consejo Nacional de Investigaciones Científicas y Técnicas, Instituto Argentino de Matemática, Buenos Aires, 1976. MR 0460947

[3] G. Eskin, Lectures on Linear Partial Differential Equations, Graduate Studies in Mathematics, 123. American Mathematical Society, Providence, RI, 2011. MR 2809923

[4] D. Gilbarg and N. S. Trudinger, Elliptic Partial Differential Equations of Second Order, Springer-Verlag, Berlin, 2001. MR 1814364

[5] F. John, Plane Waves and Spherical Means Applied to Partial Differential Equations, Interscience Publishers, New York-London, 1955. MR 0075429

[6] N. V. Krylov, Lectures on Elliptic and Parabolic Equations in Hölder Spaces, Graduate Studies in Mathematics, 12. American Mathematical Society, Providence, RI, 1996. MR 1406091

[7] M. A. Muschietti and F. Tournier, Higher order elliptic equations in half space, Rev. Un. Mat. Argentina 58 (2017), no. 2, 259-280. MR 3733207

[8] L. Nirenberg, Estimates and existence of solutions of elliptic equations, Comm. Pure Appl. Math. 9 (1956), 509-529. MR 0091402 
[9] L. Nirenberg, Remarks on strongly elliptic partial differential equations, Comm. Pure Appl. Math. 8 (1955), 649-675. MR 0075415

[10] R. Seeley, The resolvent of an elliptic boundary problem, Amer. J. Math. 91 (1969), 889-920. MR 0265764

\author{
M. A. Muschietti \\ Departamento de Matemática, UNLP, La Plata, Argentina \\ F. Tournier $\bowtie$ \\ Instituto Argentino de Matemática, CONICET, Buenos Aires, Argentina \\ fedeleti@aol.com
}

Received: June 5, 2017

Accepted: April 13, 2019 